\title{
Dust in the Wind: Composition and Kinematics of Galaxy Outflows at the Peak Epoch of Star Formation
}

\author{
Tucker Jones ${ }^{1}$ (10), Daniel P. Stark ${ }^{2}$, and Richard S. Ellis ${ }^{3}$ \\ ${ }^{1}$ Department of Physics, University of California, Davis, 1 Shields Avenue, Davis, CA 95616, USA; tdjones@ucdavis.edu \\ ${ }^{2}$ Steward Observatory, University of Arizona, 933 N Cherry Avenue, Tucson, AZ 85719, USA \\ ${ }^{3}$ Department of Physics and Astronomy, University College London, Gower Street, London WC1E 6BT, UK \\ Received 2018 May 2; revised 2018 July 2; accepted 2018 July 11; published 2018 August 23
}

\begin{abstract}
Galactic-scale outflows regulate the stellar mass growth and chemical enrichment of galaxies, yet key outflow properties such as the chemical composition and mass-loss rate remain largely unknown. We address these properties with Keck/ESI echellete spectra of nine gravitationally lensed $z \simeq 2-3$ star-forming galaxies, probing a range of absorption transitions. Interstellar absorption in our sample is dominated by outflowing material with typical velocities of $\sim-150 \mathrm{~km} \mathrm{~s}^{-1}$. Approximately $80 \%$ of the total column density is associated with a net outflow. Mass-loss rates in the low-ionization phase are comparable to or in excess of the star formation rate, with total outflow rates likely higher when accounting for ionized gas. On the order of half of the heavy element yield from star formation is ejected in the low-ionization phase, confirming that outflows play a critical role in regulating galaxy chemical evolution. Covering fractions vary and are in general non-uniform, with most galaxies having incomplete covering by the low ions across all velocities. Low-ion abundance patterns show remarkably little scatter, revealing a distinct "chemical fingerprint" of outflows. Gas-phase $\mathrm{Si} / \mathrm{Fe}$ abundances are significantly supersolar $([\mathrm{Si} / \mathrm{Fe}] \gtrsim 0.4)$, indicating a combination of $\alpha$-enhancement and dust depletion. The derived properties are comparable to the most kinematically broad, metal-rich, and depleted intergalactic absorption systems at similar redshifts, suggesting that these extreme systems are associated with galactic outflows at impact parameters conservatively within a few tens of kiloparsecs. We discuss implications of the abundance patterns in $z \simeq 2-3$ galaxies and the role of outflows at this epoch.
\end{abstract}

Key words: galaxies: evolution - galaxies: ISM

\section{Introduction}

Energetic feedback from star formation and supermassive black holes is widely regarded as a fundamentally important component of galaxy formation. Powerful feedback-driven outflows of interstellar media (ISM) are invoked to explain such properties as the galaxy stellar mass function (e.g., Bower et al. 2012), mass-metallicity relation (e.g., Dekel \& Silk 1986; Tremonti et al. 2004), and chemical enrichment of the circumgalactic and intergalactic medium (CGM and IGM; e.g., Cowie et al. 1995; Tumlinson et al. 2011). Observations of the CGM surrounding massive galaxies find that the majority of all metals ever produced in stars have been ejected from their origin galaxies (Peeples et al. 2014), presumably driven by such feedback. At low masses, stellar metallicity distributions of dwarf spheroidal galaxies show that $>90 \%-99 \%$ of their metals have been ejected (Kirby et al. 2011).

Outflows driven by star formation and active galactic nuclei (AGNs) are expected to be most influential at redshifts $z \simeq 1-3$ when these energy sources were most active (e.g., Madau \& Dickinson 2014). Indeed, early spectroscopy of Lyman-break galaxies revealed powerful galactic-scale outflows of ISM (Pettini et al. 1998), which are now known to be ubiquitous among the star-forming population at high redshifts (e.g., Shapley et al. 2003; Steidel et al. 2010; Jones et al. 2012). However, despite widespread evidence for dramatic effects of large-scale outflows, their fundamental properties remain largely undetermined. Outflow mass-loss rates, recycling rates (via so-called galactic fountains), and chemical compositions are critically important parameters yet they vary widely among different galaxy formation models and cosmological simulations (e.g., Davé et al. 2011; Gibson et al. 2013). While there is general agreement about the broad effects of feedback-driven outflows on galaxies and the IGM, precise knowledge of their physical properties is needed for cosmological galaxy formation models to gain testable predictive power.

Observational probes of outflow properties largely come from two complementary approaches. The spectra of background sources provide information on the distribution of gas seen in absorption as a function of projected impact parameter. Generally, only one background sightline is available for a given galaxy, but statistical ensembles have revealed the average radial absorption profiles for well-defined galaxy populations (Ménard et al. 2010; Steidel et al. 2010; Tumlinson et al. 2011; Rudie et al. 2012; Turner et al. 2014). These data show that the majority of gas absorption is found relatively close to galaxies (within a few tens of kiloparsecs), while the metal-enriched CGM extends to $\gtrsim 100 \mathrm{kpc}$. This is supported by spatial mapping of outflows in a few rare cases at $z \gtrsim 1$ (Martin et al. 2013; Finley et al. 2017); more examples may soon become available thanks to sensitive integral field spectrographs such as MUSE and KCWI. The other common probe is a "down-the-barrel" view of outflowing material detected as blueshifted absorption in galaxy spectra. This provides good kinematic data but little or no information on the radial profile relative to the host galaxy.

A major limitation of current data is that spectroscopic measurements sampling the bulk of the material-namely, down the barrel or at very close impact parameters - are almost exclusively limited to the strongest absorption features. These transitions are typically saturated such that their equivalent widths encapsulate the gas kinematics and geometric covering 
fraction (e.g., Steidel et al. 2010; Jones et al. 2012, 2013; Leethochawalit et al. 2016) but not the column density. In essence, there is little information about the actual mass flux or chemical composition which are critical for understanding how outflows regulate galaxy growth and the CGM+IGM. The simple reason for this shortcoming is that typical galaxies are too faint for the detailed study of optically thin absorption lines. To date, this has been overcome only with gravitationally lensed galaxies whose apparent luminosities are magnified by factors $\gtrsim 10 \times$. This was first accomplished by Pettini et al. (2002), who measured column densities and kinematics for a range of ionic species in the spectacular lensed galaxy cB58 at $z=2.7$, revealing outflows with chemical enrichment to a few tenths of the solar value and mass-loss rates likely exceeding the star formation rate (SFR). While only two additional $z>2$ lensed galaxies have been studied in such detail (Quider et al. 2009; Dessauges-Zavadsky et al. 2010), enlarged samples of bright gravitationally lensed galaxies (Stark et al. 2013; Rigby et al. 2018) now provide the opportunity for high-quality spectroscopy of a representative population at high redshifts.

This paper is concerned with characterizing the typical physical properties of galaxy ISM and outflows during the peak periods of their star formation at $z \simeq 2-3$. Our goal is to obtain measurements similar to those in the pioneering study of Pettini et al. (2002) for a statistically interesting sample representative of the star-forming population, for which thousands of spectra exist but only the strongest ISM features have been characterized thus far. Our study leverages the Cambridge And Sloan Survey Of Wide ARcs in the skY (CASSOWARY, which we abbreviate as CSWA; Belokurov et al. 2007, 2009). The CSWA survey has identified more than 100 candidate strong gravitational lens systems in Sloan Digital Sky Survey imaging, with typical lensed galaxy AB magnitudes of 20-21 at optical wavelengths. We have conducted follow-up campaigns with various telescopes to spectroscopically confirm the nature of these systems (Stark et al. 2013). The resulting catalog represents a collection of galaxies that are characteristic of the population at their redshifts yet are among the brightest examples on the sky thanks to strong lensing magnification. As such, these are ideal sources for deep follow-up spectroscopy. We have previously utilized this sample to measure kinematic properties and to spatially map nebular metallicities using integral field spectroscopy, demonstrating the power of lensing systems to better understand the complex galaxy formation process (Jones et al. 2013; Leethochawalit et al. 2016). We now present a study of the ISM and outflow properties for nine galaxies at $z=1.4-2.9$, in total quadrupling the available sample of quality absorption line spectroscopy at these redshifts.

The paper is structured as follows. The spectroscopic observations are described in Section 2. Measurements of gas covering fractions and column densities are presented in Section 3. We present our analyses of the gas kinematics in Section 4, and chemical properties including intrinsic abundances, dust depletion, and ionization state in Section 5. We discuss the implications of our findings for the galaxy population and its evolution in Section 6, and conclude with a summary of the main results in Section 7. Throughout this work, we adopt vacuum wavelengths from the NIST Atomic Spectra Database (Kramida et al. 2016) and oscillator strengths $f$ from the compilations of Morton $(2000,2003)$, with the exception of $\mathrm{Ni}$ II $\lambda 1317$, where we use $f=0.07786$
(Morton 1991). Solar abundances refer to the photospheric values given by Asplund et al. (2009), and literature measurements are adjusted to this solar scale as needed. Where necessary we adopt a flat $\Lambda$ CDM cosmology with $H_{0}=$ $70 \mathrm{~km} \mathrm{~s}^{-1} \mathrm{Mpc}^{-1}, \Omega_{\Lambda}=0.7$, and $\Omega_{M}=0.3$.

\section{Observations and Data Reduction}

All objects were observed with the Echelle Spectrograph and Imager (ESI; Sheinis et al. 2002) on the Keck II telescope. Data were obtained on two observing runs in 2012 November and 2013 March. A summary of the targets and exposure times is given in Table 1. Spectra were taken in echellete mode with a 0 " 75 slit, resulting in a spectral resolution $R=6300$ measured from sky lines (velocity resolution $=48 \mathrm{~km} \mathrm{~s}^{-1} \mathrm{FWHM}$ ). Data were reduced using the IDL-based ESIRedux code written by J. X. Prochaska. We first apply a bias subtraction and flat-field correction to all frames using calibration data taken on the same night. Sky subtraction is performed using polynomial and spline fits to the corrected $2 \mathrm{D}$ spectra. We extract the 1D spectrum of each object using a boxcar aperture, based on the observed spatial extent of each target along the slit. In some cases, we observe multiple lensed images of the same target and extract their traces separately. Individual exposures are combined by first normalizing all extracted spectra to the mean continuum level and then taking the weighted mean with cosmic-ray rejection. Where applicable, spectra of multiple images are then combined. We normalize the spectra to a running median of the continuum level over regions free of strong spectral features. The continuum level is determined to a precision of $\sim 2 \%(1 \sigma)$ for the typical signal-to-noise ratio $(\mathrm{S} / \mathrm{N})$ of 10 per resolution element. This does not substantially contribute to uncertainty in column density measurements, which are instead dominated by the limited $\mathrm{S} / \mathrm{N}$ of individual absorption lines. We further confirm that the continuum normalization does not introduce large systematic errors, as we find consistent column densities from different transitions of the same ion (Section 3.1.1). Visual inspection shows that the continuum level behaves well near intrinsic absorption and emission features in the galaxy spectra, which are masked from the continuum estimate. However, the continuum is less reliable near strong telluric features, including sky emission lines. Features that are visibly affected by telluric absorption or sky-line residuals are therefore excluded from analysis.

The spectra of each object are shown in Figure 1. In most cases, the stellar continuum is detected at $\gtrsim 10 \sigma$ per resolution element in good sky regions (Table 1). In three cases, we smooth the data to improve the precision of absorption line measurements. This results in a final FWHM spectral resolution of $85 \mathrm{~km} \mathrm{~s}^{-1}$ for CSWA 141, $102 \mathrm{~km} \mathrm{~s}^{-1}$ for CSWA 40, and $157 \mathrm{~km} \mathrm{~s}^{-1}$ for CSWA 2, improving the $\mathrm{S} / \mathrm{N}$ to $\sim 6-8$ in each case.

\subsection{Systemic Redshifts}

Systemic redshifts are measured for each source from spectral features that originate from either stellar photospheric absorption or nebular emission in $\mathrm{H}$ II regions. The features used to derive redshifts are listed in Table 2, and we give details for individual sources in the Appendix. We also list the redshifts derived for $\mathrm{Fe} \mathrm{II}^{*}$ and $\mathrm{Si} \mathrm{II}^{*}$ fine-structure emission lines, which typically peak near the systemic velocity, although these features arise predominantly in outflowing gas 
Table 1

Observing Log

\begin{tabular}{|c|c|c|c|c|c|c|c|}
\hline ID & Dates Observed & $z^{\mathrm{a}}$ & R.A. & Decl. & $\mathrm{PA}$ & $t_{\exp }(\mathrm{ks})$ & $\overline{\mathrm{S} / \mathrm{N}^{\mathrm{b}}}$ \\
\hline CSWA 141 & 2012 Nov 8 & $1.425194 \pm 0.000003$ & $08: 46: 47.53$ & $+04: 46: 09.3$ & 190 & 5.2 & 4.5 \\
\hline CSWA 103 & 2012 Nov $8-10$ & $1.95978 \pm 0.00008$ & 01:45:04.38 & $-04: 55: 50.8$ & 115 & 25 & 11.5 \\
\hline CSWA 19 & 2012 Nov $9-10$ & $2.03237 \pm 0.00011$ & 09:00:02.80 & $+22: 34: 07.1$ & 86 & 20 & 18.3 \\
\hline CSWA 40 & 2013 Mar 5-6 & $2.18938 \pm 0.00007$ & 09:52:40.29 & $+34: 34: 39.2$ & 70 & 16.2 & 5.4 \\
\hline CSWA 2 & 2013 Mar 5 & $2.19677 \pm 0.00007$ & $10: 38: 41.88$ & $+48: 49: 22.4$ & 17 & 7.2 & 3.0 \\
\hline CSWA 128 & 2012 Nov $8-10$ & $2.22505 \pm 0.00003$ & $19: 58: 35.44$ & $+59: 50: 52.2$ & 60 & 16.3 & 15.4 \\
\hline CSWA 164 & 2012 Nov $8-10$ & $2.51172 \pm 0.00007$ & $02: 32: 49.93$ & $-03: 23: 25.8$ & 158 & 28 & 15.3 \\
\hline CSWA 39 & 2013 Mar 5-6 & $2.76223 \pm 0.00008$ & $15: 27: 45.16$ & $+06: 52: 19.6$ & 105 & 18 & 10.7 \\
\hline CSWA 38 & 2013 Mar 6 & $2.92556 \pm 0.00007$ & $12: 26: 51.48$ & $+21: 52: 17.9$ & 130 & 10.8 & 9.8 \\
\hline
\end{tabular}

Notes.

a Adopted systemic redshift and formal $1 \sigma$ uncertainty.

${ }^{\mathrm{b}}$ Continuum signal-to-noise ratio per $1.0 \AA$ resolution element at $\lambda=6200 \AA$.

(e.g., Prochaska et al. 2011; Jones et al. 2012) and thus are not expected to trace the stellar kinematics. The root-mean-square scatter between fine-structure emission line centroids and the adopted systemic redshift is $31 \mathrm{~km} \mathrm{~s}^{-1}$ with mean and median offset $<10 \mathrm{~km} \mathrm{~s}^{-1}$. While the fine-structure redshifts have larger scatter than true systemic features, they are nonetheless more accurate than other methods used to estimate redshifts when no other systemic features are available (e.g., Ly $\alpha$ emission or interstellar absorption lines; Steidel et al. 2010).

Redshift uncertainties in Table 2 correspond to a formal $1 \sigma$ uncertainty in the centroid of Gaussian fits to the features. In general, these fits have reduced- $\chi^{2}$ values of order unity, indicating that the estimated measurement errors are accurate. However, the dispersion in redshift measured from different spectral features is somewhat larger than the statistical uncertainty. This could be caused by the different physical origins of the various features, spurious fits of noisy features, underestimation of the true uncertainty, or a systematic error in the wavelength calibration of $\sigma(\lambda) / \lambda \simeq 5 \times 10^{-5}$ in different echelle orders. In any case, the adopted redshifts are accurate to $\lesssim 20 \mathrm{~km} \mathrm{~s}^{-1}$ in velocity across the observed ESI spectra.

\subsection{Demographic Properties of the Sample}

Galaxies in our sample are representative of the intermediate-mass star-forming population studied by other large surveys at $z \simeq 2-3$ (e.g., Sanders et al. 2015; Steidel et al. 2016; Wuyts et al. 2016). At present, we have obtained robust stellar masses and SFRs for seven galaxies in our sample with near-IR imaging (all except CSWA 38 and 164; R. Mainali et al. 2018, in preparation). The sample median properties from SED fitting with an assumed constant star formation history, metallicity of 0.2 solar, and a Chabrier IMF are $\log M_{\star} / M_{\odot}=9.8, \mathrm{SFR}=$ $13 M_{\odot} \mathrm{yr}^{-1}$, and $\mathrm{sSFR}=2 \mathrm{Gyr}^{-1}$.

\subsection{Intervening Absorption Systems}

In addition to the ISM of our target galaxies, the spectra are sensitive to absorption arising from intervening material at lower redshift along the line of sight. Figure 2 shows three such systems at $z \simeq 1-2$ detected in the ESI spectra. A detailed census of their properties is beyond the scope of this paper. For our purposes we are interested in whether any absorption lines used in this work are affected by intervening systems. Si IV $\lambda 1402$ in CSWA 128 and Si II $\lambda 1260$ in CSWA 39 are blended with strong features arising from lower redshift systems (Figure 2), and we therefore do not use these in subsequent analysis. We find no other cases where intervening absorbers significantly affect the results.

\section{Gas Covering Fractions and Column Densities}

The goals of this work demand measurements of column density from a variety of ions. Given the extended nature of the continuum source, emergent absorption line profiles may arise from a blend of components at different velocities and spatial positions. We are most interested in ions that are physically associated such that their intrinsic abundance ratios can be determined. We thus focus on the "low ions," which are predominantly associated with a common $\mathrm{H}$ I gas phase, arise in the same absorption components (e.g., Wolfe \& Prochaska 2000; Werk et al. 2013), and for which multiple elements are probed. Wherever possible we also seek to determine the contribution of different ionization states to the total mass and elemental abundances.

We make the simplifying assumptions that at a given velocity, the medium in which absorption from a given ion arises is characterized by (1) uniform chemical composition, (2) uniform column density, and (3) finite spatial extent, such that a fraction $f_{c}$ of the stellar continuum is covered by the absorbing medium. Following Jones et al. (2013), we then calculate ion column densities and covering fractions from measured absorption line profiles as follows. The residual intensity $I$ of an absorption line is given by

$$
\frac{I}{I_{0}}=1-f_{c}\left(1-e^{-\tau}\right),
$$

where $I_{0}$ is the continuum level. The optical depth $\tau$ is in turn related to column density by

$$
\tau=f \lambda \frac{\pi e^{2}}{m_{e} c} \times N=\frac{f \lambda}{3.768 \times 10^{14}} \times N,
$$

where $f$ is the ion oscillator strength, $\lambda$ is the rest-frame wavelength of the transition expressed in $\AA$, and $N$ is the ion column density in $\mathrm{cm}^{-2}\left(\mathrm{~km} \mathrm{~s}^{-1}\right)^{-1}$. Combining Equations (1) and (2) yields an expression for $I$ as a function of $f_{c}$ and $N$. In the following analysis, we will treat all variables as functions of velocity, i.e., $N(v)$ and $f_{c}(v)$.

In cases where the data include two or more transitions of the same ion, from the same ground state, with different values of $f \lambda$, it is possible to solve Equations (1) and (2) for $N$ and $f_{c}$. Most spectra discussed here cover multiple transitions of Si II, 


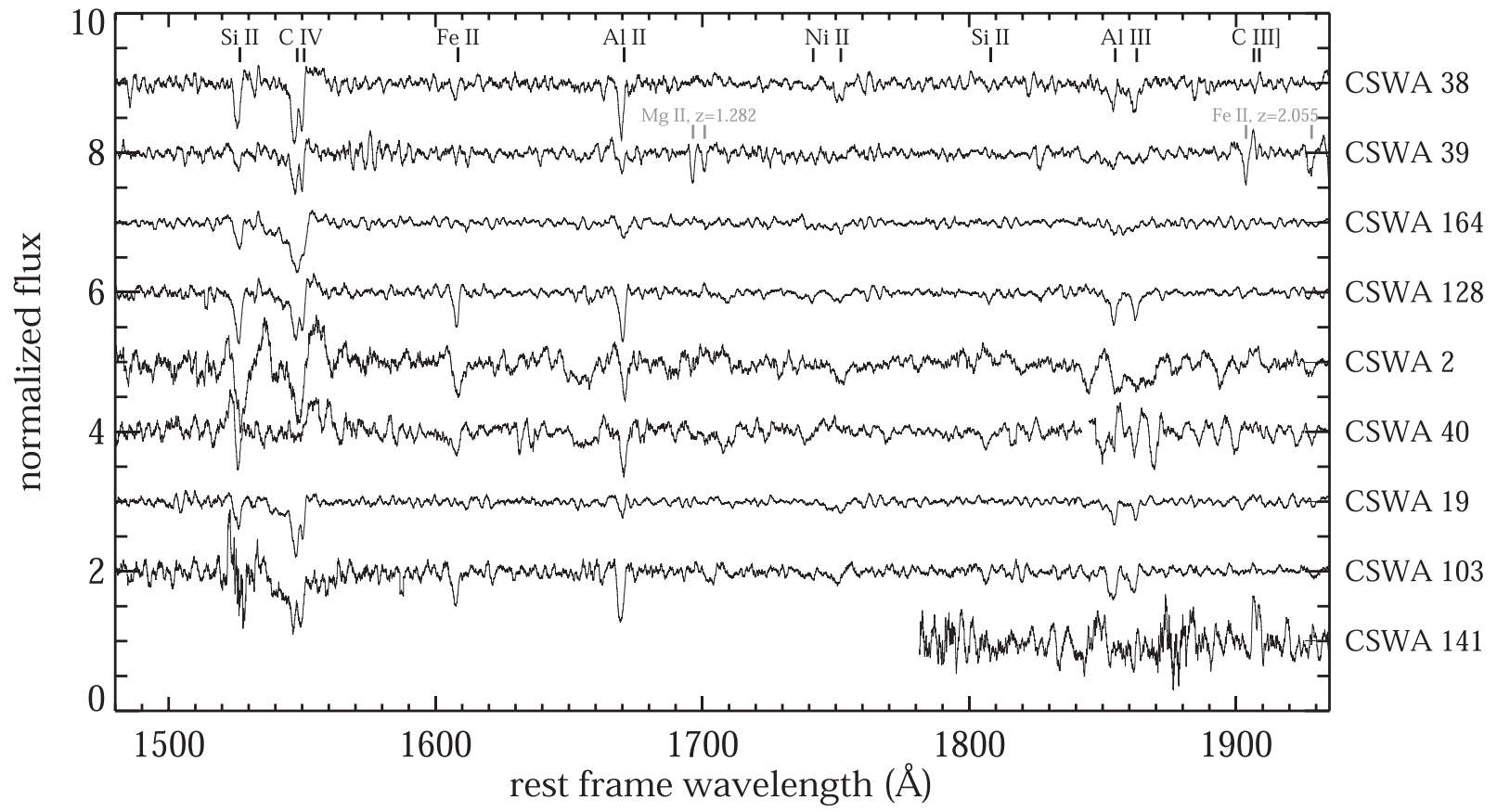

Figure 1. Spectra of the nine galaxies in the ESI sample in the rest-frame wavelength range 1500-1900 $\AA$, normalized and offset for clarity, ordered from highest (top) to lowest redshift. In the case of CSWA 141, we show only the spectrum redward of $4300 \AA$ in the observed frame. The spectra are smoothed to $\sim 250 \mathrm{~km} \mathrm{~s}^{-1} \mathrm{FWHM}$ resolution for display purposes. Several prominent features are labeled at the top. We also label strong lines from two intervening absorption systems identified in the spectrum of CSWA 39. Significant dispersion can be seen in the equivalent width of features such as Si II $\lambda 1526$ and Al II $\lambda 1670$, which are typically optically thick and therefore trace the covering fraction and velocity profile of low-ionization gas. Weaker lines such as Si II $\lambda 1808$ and Ni II $\lambda 1741,1751$ are additionally sensitive to the gas column density.

Table 2

Systemic Redshift Features

\begin{tabular}{|c|c|c|}
\hline$\overline{\mathrm{ID}}$ & Feature & $z$ \\
\hline \multirow[t]{2}{*}{ CSWA 141} & [O II] $\lambda \lambda 3727,9$ & $1.425194 \pm 0.000003^{\mathrm{a}}$ \\
\hline & C III] $\lambda \lambda 1907,9$ & $1.425248 \pm 0.000033$ \\
\hline \multirow[t]{3}{*}{ CSWA 103} & C III] $\lambda \lambda 1907,9$ & $1.95978 \pm 0.00008^{\mathrm{a}}$ \\
\hline & $\mathrm{Fe}$ II $^{*} \lambda 2365$ & $1.95957 \pm 0.00008$ \\
\hline & $\mathrm{Fe} \mathrm{II}^{*} \lambda 2612$ & $1.95987 \pm 0.00004$ \\
\hline \multirow[t]{4}{*}{ CSWA 19} & C III] $\lambda \lambda 1907,9$ & $2.03237 \pm 0.00011^{\mathrm{a}}$ \\
\hline & $\mathrm{Fe} \mathrm{II}^{*} \lambda 2365$ & $2.03217 \pm 0.00009$ \\
\hline & Si III $\lambda 1417$ & $2.03219 \pm 0.00009$ \\
\hline & C III $\lambda 2297$ & $2.03268 \pm 0.00008$ \\
\hline \multirow[t]{2}{*}{ CSWA 40} & stellar absorption $^{\mathrm{b}}$ & $2.18938 \pm 0.00007^{\mathrm{a}}$ \\
\hline & $\mathrm{Si} \mathrm{II} * \lambda 1533$ & $2.18926 \pm 0.00007$ \\
\hline \multirow[t]{2}{*}{ CSWA 2} & C III] $\lambda \lambda 1907,9$ & $2.19677 \pm 0.00007^{\mathrm{a}}$ \\
\hline & $\mathrm{Fe} \mathrm{II}^{*} \lambda 2396$ & $2.19625 \pm 0.00014$ \\
\hline CSWA 128 & O III] $\lambda \lambda 1661,6$ & $2.22505 \pm 0.00003^{\mathrm{a}}$ \\
\hline \multirow[t]{3}{*}{ CSWA 164} & stellar absorption $^{\mathrm{b}}$ & $2.51172 \pm 0.00007^{\mathrm{a}}$ \\
\hline & C III] $\lambda \lambda 1907,9$ & $2.51207 \pm 0.00016$ \\
\hline & Si II* $\lambda 1533$ & $2.51222 \pm 0.00006$ \\
\hline \multirow[t]{4}{*}{ CSWA 39} & C III] $\lambda \lambda 1907,9$ & $2.76223 \pm 0.00008^{\mathrm{a}}$ \\
\hline & O III] $\lambda \lambda 1661,6$ & $2.76204 \pm 0.00007$ \\
\hline & Si II* $\lambda 1533$ & $2.76244 \pm 0.00006$ \\
\hline & stellar absorption $^{\mathrm{c}}$ & $2.76238 \pm 0.00007$ \\
\hline \multirow[t]{3}{*}{ CSWA 38} & stellar absorption $^{\mathrm{c}}$ & $2.92556 \pm 0.00007^{\mathrm{a}}$ \\
\hline & C III] $\lambda \lambda 1907,9$ & $2.92588 \pm 0.00019$ \\
\hline & Si II* $\lambda 1533$ & $2.92599 \pm 0.00007$ \\
\hline
\end{tabular}

\section{Notes.}

adopted systemic redshift.

${ }^{\mathrm{b}}$ Simultaneous fit to stellar photospheric absorption features Si III $\lambda 1294$, Si III $\lambda 1417, \mathrm{~S} \vee \lambda 1501, \mathrm{~N}$ IV $\lambda 1718$, and C III $\lambda 2297$.

${ }^{\mathrm{c}}$ Simultaneous fit to Si III $\lambda 1294$, Si III $\lambda 1417, \mathrm{~S}$ V $\lambda 1501$, and N IV $\lambda 1718$.
Fe II, Ni II, Al III, Si IV, and C IV. Of these, Si II and Fe II typically provide the most robust results as there are several transitions covering a wide range of $f \lambda$. Figure 3 illustrates how various transitions of $\mathrm{Si}$ II and Fe II provide excellent constraints over nearly four orders of magnitude in column density from $N \approx 5 \times 10^{10}$ to $2 \times 10^{14} \mathrm{~cm}^{-2}\left(\mathrm{~km} \mathrm{~s}^{-1}\right)^{-1}$. An example of multiple $\mathrm{Si}$ II transitions in one spectrum is shown in Figure 4.

\subsection{Low-ionization Gas}

This paper focuses largely on low-ionization species (low ions), defined as having ionization potentials straddling 1 Rydberg and which are typically the dominant ionization state in $\mathrm{H}$ I gas. These include C II, O I, Al II, Si II, Fe II, Ni II, and $\mathrm{Zn}$ II, which are relevant for this work. Low ions from different elements are assumed to be cospatial, and in many cases we can verify that $\mathrm{Si}$ II and $\mathrm{Fe}$ II give consistent results for $f_{c}$ (e.g., Figure 5). We note that Si II, Fe II, Ni II, and Zn II in particular have nearly identical ionization potentials (within 1-2 eV) and therefore should be strongly associated. Wherever possible we determine $f_{c}$ for low ions using three different estimates: $f_{c}\left(\mathrm{Si}\right.$ II), $f_{c}(\mathrm{Fe}$ II), and a weighted mean of saturated absorption lines. Our methods are essentially identical to those used in Jones et al. (2013) and Leethochawalit et al. (2016), which we briefly summarize. For $\mathrm{Si}$ II and Fe II, we find the values of $N$ and $f_{c}$ that minimize the least-squares residual,

$$
\chi^{2}=\sum\left(I_{\mathrm{obs}}-I_{N, f_{c}}\right)^{2} / \sigma_{\mathrm{obs}}^{2},
$$

in each velocity bin, summing over all available transitions (e.g., Figure 4). For the weighted-mean approach, we approximate 


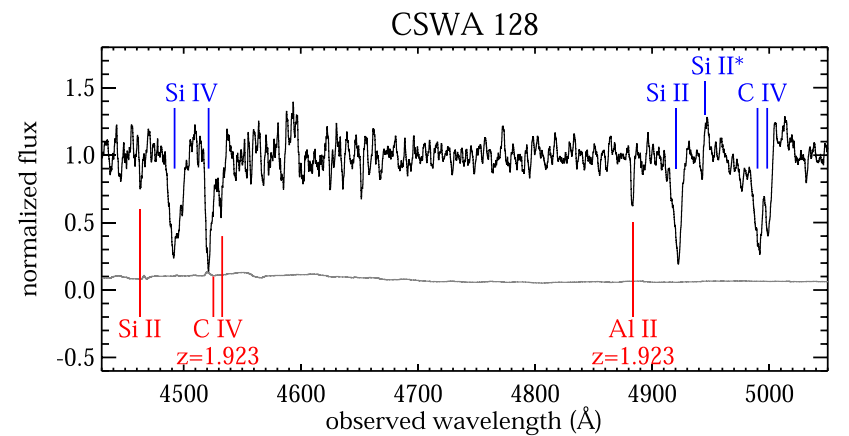

CSWA 39

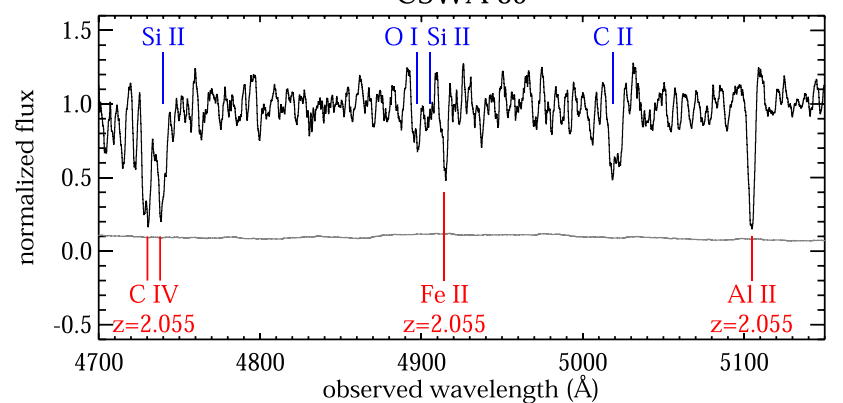

CSWA 38

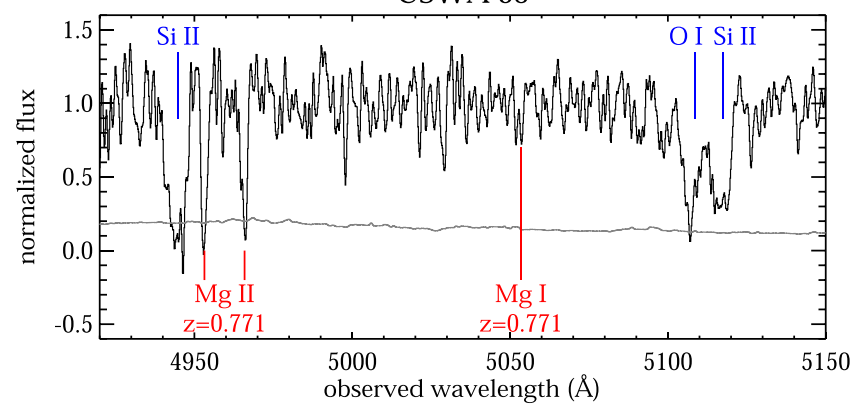

Figure 2. Intervening absorption systems in the ESI spectroscopic sample. Absorption lines intrinsic to the galaxy's interstellar and circumgalactic medium are labeled above in blue, with intervening absorption lines labeled below in red. Error spectra are shown in gray. Absorption from foreground systems can be problematic by blending with intrinsic features in the galaxy spectrum, such as Si IV $\lambda 1402$ in CSWA 128 (blended with intervening C IV at $z=1.923$ ) and Si II $\lambda 1260$ in CSWA 39 (blended with intervening $\mathrm{C}$ IV at $z=2.055)$. These blends are excluded from analysis.

Equation (1) as

$$
f_{c}=1-I / I_{0}
$$

and average the subset of Si II $\lambda \lambda \lambda 1260,1304,1526$; O I $\lambda 1302$; C II $\lambda 1334$; Al II $\lambda 1670$; Fe II $\lambda 2382$; and $\mathrm{Mg}$ II $\lambda \lambda 2796,2803$ profiles that appear saturated based on visual inspection. Blended transitions are excluded (e.g., blended regions of O I $\lambda 1302$ and Si II $\lambda 1304)$. Transitions are considered saturated if their optical depths $\tau \gtrsim 3$ such that Equation (4) is a good approximation. For example, cases where the Si II $\lambda \lambda \lambda 1260,1304,1526$ profiles are identical to within $\lesssim 5 \%$ indicate that all three are saturated (see Figure 3). In the example shown in Figure 4, Si II $\lambda 1260$ is saturated and used to determine $f_{c}$, whereas the $\lambda 1304$ and $\lambda 1526$ transitions have $\tau \sim 1$ and are not used.

In general, the three methods of deriving low-ion covering fractions are in good agreement. Figure 5 shows the results for CSWA 128 as an example. The weighted mean of saturated
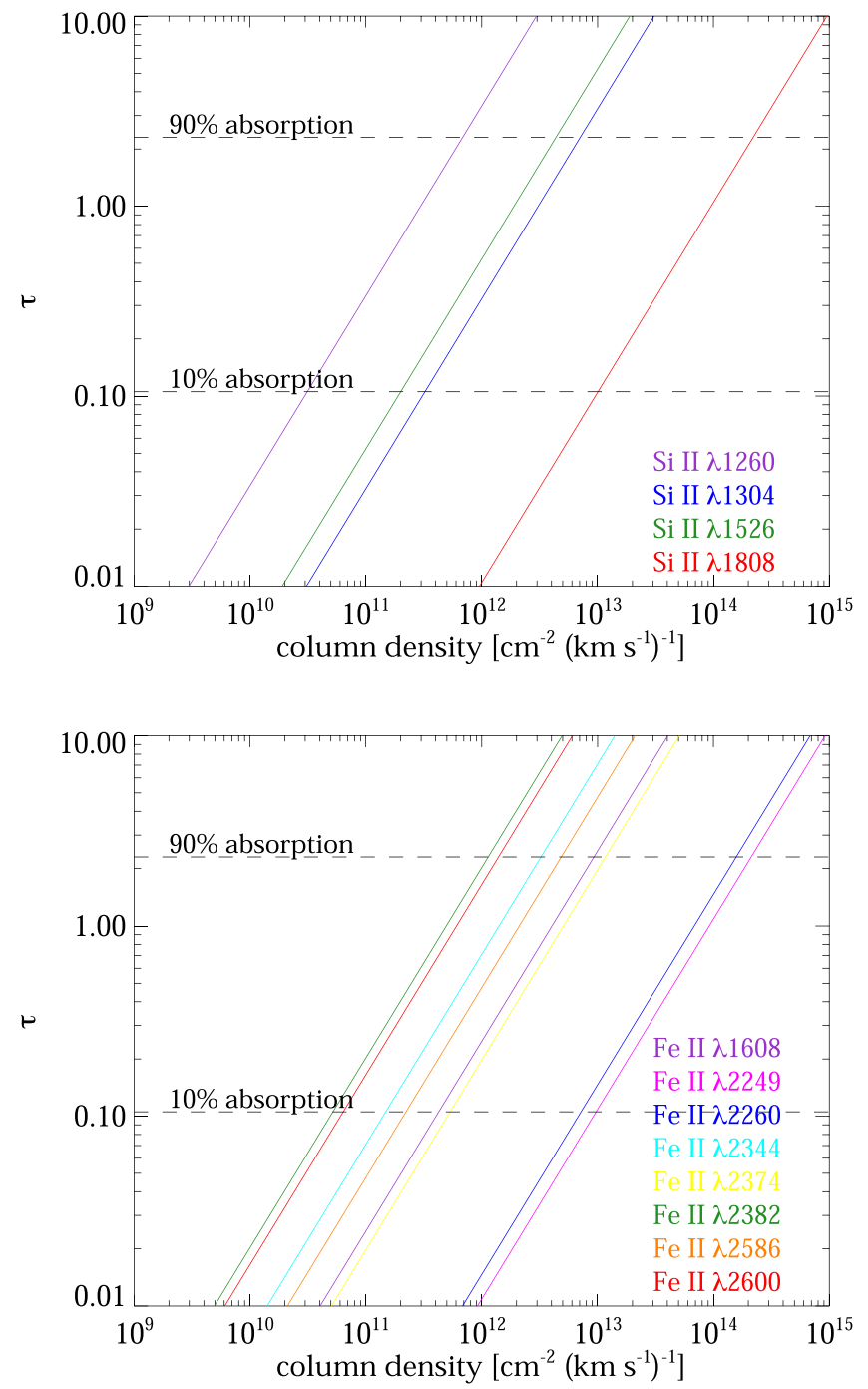

Figure 3. Optical depth $\tau$ as a function of column density for $\mathrm{Si}$ II and Fe II transitions observed in the ESI spectra. The range of $f \lambda$ values enables robust measurements of column density over several orders of magnitude. For the ESI spectra, column density measurements generally require at least one optically thin line for which the absorption depth is $\lesssim 50 \%$ of the continuum $(\tau \lesssim 0.7)$, combined with an optically thick transition of $\tau \gtrsim 3$ to determine the covering fraction.

transitions consistently has the lowest uncertainty and so we adopt this as the best measurement of $f_{c}$ (as done in Jones et al. 2013; Leethochawalit et al. 2016). Another reason for using saturated transitions is that Equations (1) and (2) cannot easily distinguish between cases of low $N$ and high $f_{c}$, or high $N$ with low $f_{c}$, when absorption lines are weak. This is especially evident in Figure 5, where $f_{c}$ (Fe II) shows increased uncertainty (evidenced by large scatter) but with no strong systematic difference. Likewise, $f_{c}(\mathrm{Si}$ II) shows large scatter where absorption is weak and excellent agreement with saturated transitions at velocities where $f_{c}$ is unambiguously high $(\gtrsim 0.5)$. The weighted mean approach breaks the $N-f_{c}$ degeneracy by assuming low $f_{c}$, which is thought to be the solution in such cases (Pettini et al. 2002; Quider et al. 2010; Jones et al. 2013).

We are now in a position to summarize the covering fraction and column density measurements of low-ion species that we will use throughout this work. Figure 6 shows $f_{c}$ as a function of velocity derived from the weighted mean method. These covering fraction profiles are used to calculate low-ion column 

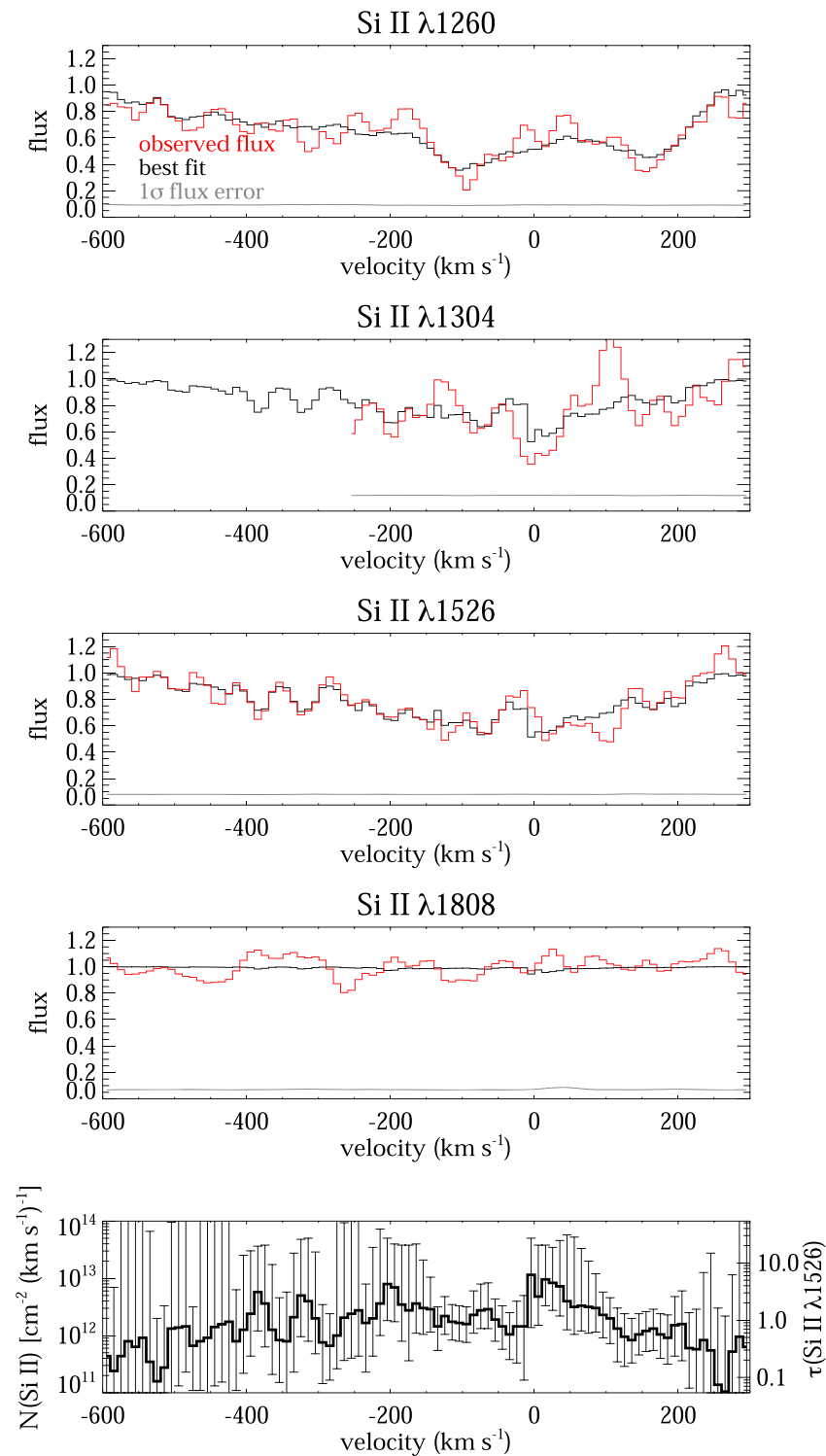

Figure 4. Column density of Si II constrained from multiple transitions in the spectrum of CSWA 164. Four transitions of Si II with different oscillator strengths are available in the spectrum. Profiles of each transition are shown in red (excluding the region where Si II $\lambda 1304$ is blended with O I $\lambda 1302$ ). Si II column density is derived at each velocity bin from a joint fit to all transitions, with the covering fraction profile fixed to an average of the saturated transitions (shown in Figure 6). The best-fit column density profile and its uncertainty are shown in the bottom panel. Line profiles corresponding to the best-fit column density and assumed covering fraction are shown as black lines in the upper panels. In this case, the $\lambda 1260$ transition is optically thick and therefore traces the covering fraction of low-ionization gas. The data are inconsistent with a uniform covering fraction.

densities from unsaturated absorption lines via Equations (1) and (2). Physically, the resulting $N(v)$ corresponds to a column density that covers an areal fraction $f_{c}(v)$ of the target galaxy, while in our formalism the remaining $1-f_{c}$ has zero column density. For this work, we are interested in the aggregate galaxy-integrated column density, defined by integrating the product of $N$ and $f_{c}$ over velocity:

$$
N_{\mathrm{tot}}=\int N f_{c} d v
$$

Effectively, $N_{\text {tot }}$ corresponds to the average column density that would be measured from an ensemble of point sources in the

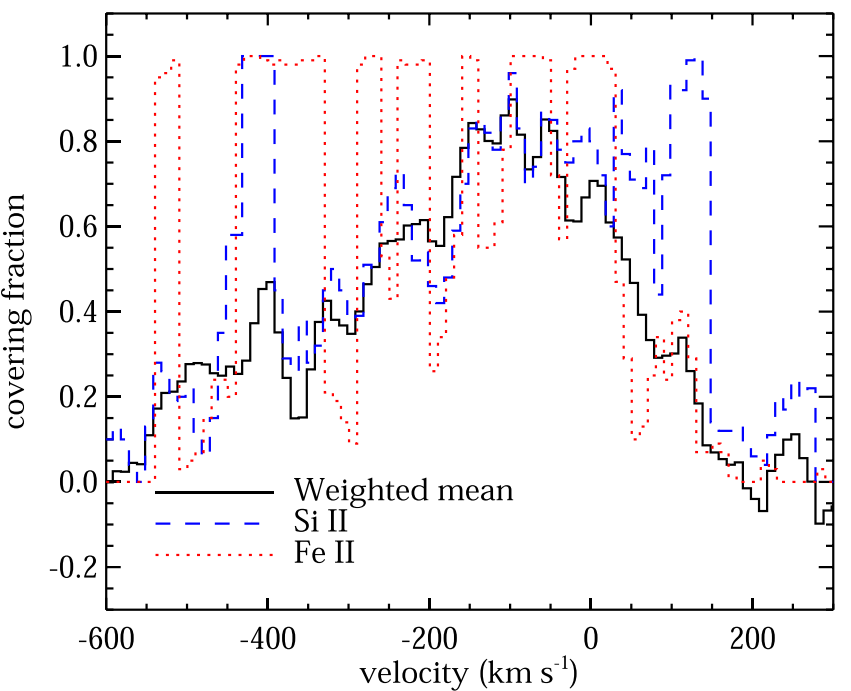

Figure 5. Low-ion covering fraction of CSWA 128 from three different methods. The covering fractions of Si II and Fe II are derived as best-fit solutions to Equations (1) and (2). The solid black line is a weighted mean of various saturated transitions as described in the text. All three methods are in good agreement within the uncertainties, which are smallest for the weighted mean method.

galaxy. This quantity has an additional advantage that in the limiting case of optically thin transitions $\tau \ll 1$, Equation (1) simplifies to

$$
N \propto \tau \propto f_{c}^{-1} .
$$

The product $N f_{c} \propto N_{\text {tot }}$ is thus approximately independent of $f_{c}$ for weak lines such as Si II $\lambda 1808$, Fe II $\lambda 2249$, and various $\mathrm{Ni}$ II transitions. We report integrated column density measurements $N_{\text {tot }}$ and corresponding velocity ranges (i.e., bounds of the integral: $\Delta v$ ) in Table 3 for transitions most relevant for this work. ${ }^{4}$ Velocity ranges are set to encompass the regions of high $f_{c}$ where absorption is well detected. We do not integrate Equation (5) over regions of weaker absorption (i.e., $f_{c} \approx 0$ ) as this would essentially add noise and could introduce systematic error because of the $f_{c}-N$ degeneracy. The adopted velocity ranges encompass a median $73 \%$ of the velocity-integrated covering fraction, varying from $63 \%$ to $85 \%$ for individual sources (except CSWA 141, where the fraction is likely $<50 \%) . N_{\text {tot }}$ values in Table 3 are therefore expected to be within $\sim 0.1$ dex of the total (integrated across all velocities), and we emphasize that the abundance ratios are robust. These low-ion covering fractions and abundance ratios derived via $N_{\text {tot }}$ constitute the basis of most results discussed in this work.

\subsubsection{Joint Fits to Multiple Transitions}

In cases where multiple transitions of a low ion are observed in the spectra, we can jointly analyze all transitions to improve the precision of column density measurements. At each velocity, we determine the best-fit $N$ via Equation (3), with $f_{c}$ fixed to the values from saturated transitions (Figure 6). Resulting $N_{\text {tot }}$ values for Si II, Fe II, and Ni II are given in Table 4 along with the transitions used. Here, $N_{\text {tot }}$ is integrated

\footnotetext{
4 In some cases, we are interested in strong transitions with $\tau \gg 1$ whose column densities cannot be reliably determined due to saturation. We instead report conservative lower limits corresponding to $\tau \geqslant 2$.
} 

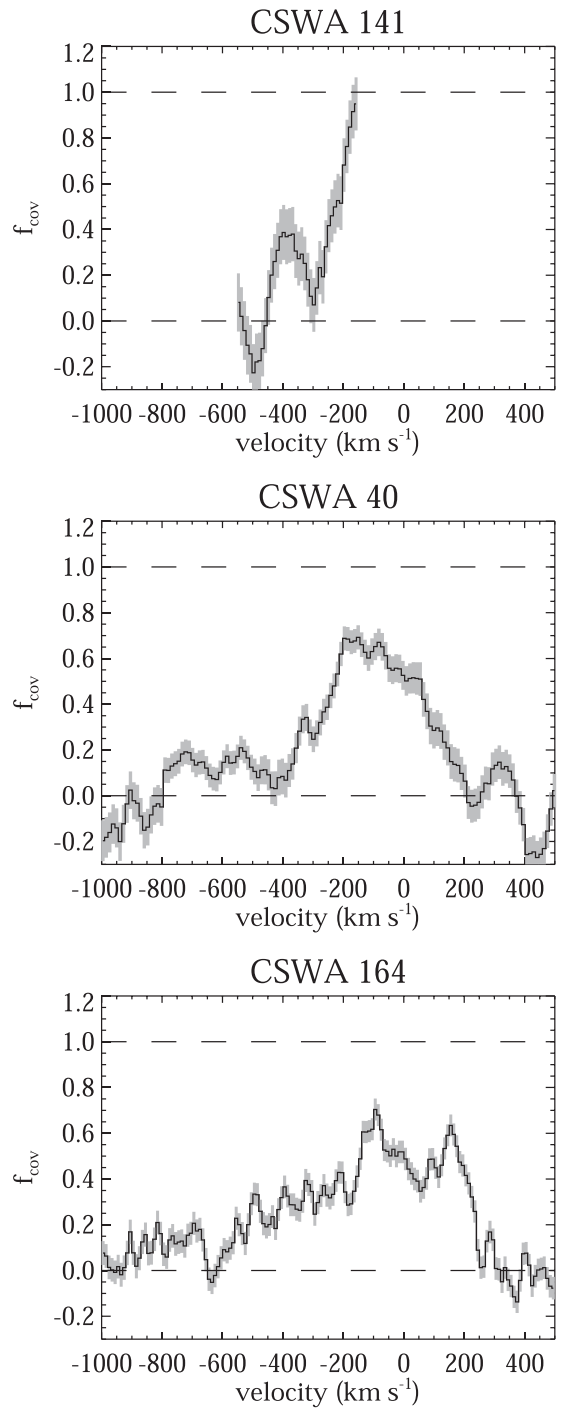
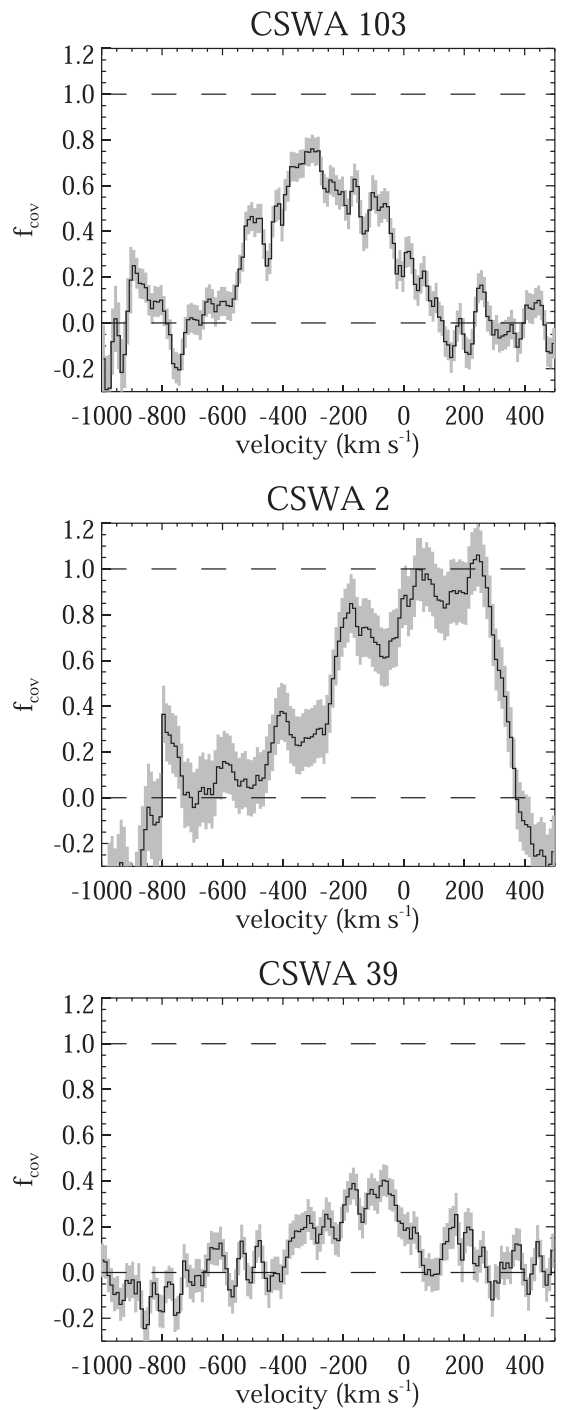
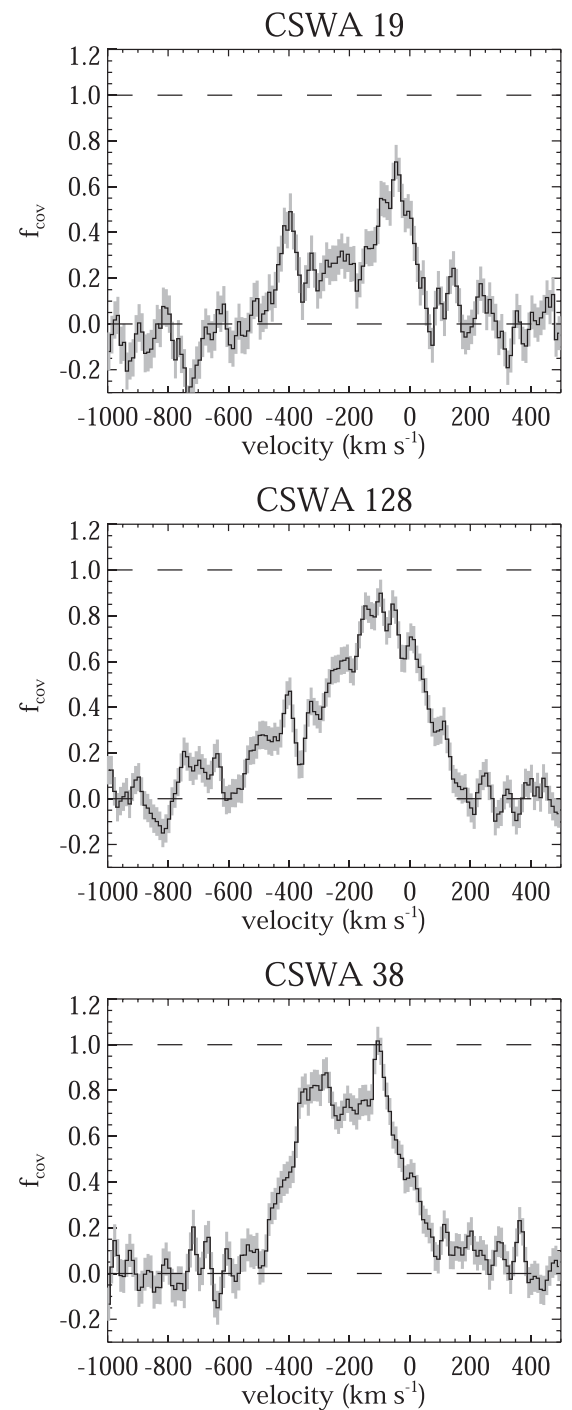

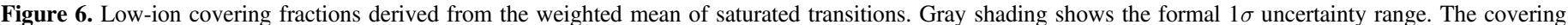
fraction of CSWA 141 is derived from Mg II and is valid only over the region shown; other velocities are contaminated by strong emission line filling.

over the same velocity range as for individual transitions to facilitate direct comparison. $N_{\text {tot }}$ values from different transitions of the same ion are in reasonable agreement (within $2 \sigma$ in nearly all cases) given the measurement uncertainties. In contrast, adopting $f_{c}=1$ would give discrepant results, confirming the non-uniform covering fractions.

Figure 4 shows an example of this method applied to Si II in CSWA 164. Various Si II transitions probe a range of optical depths $\tau \ll 1(\lambda 1808), \tau \sim 1(\lambda 1304, \lambda 1526)$, and $\tau \gg 1$ $(\lambda 1260)$. The joint analysis of all transitions enables good measurements of $N(v)$. Relative to the best-fit value, lower bounds are tightly constrained by strong lines with $\tau \gtrsim 1$ while upper bounds are subject to larger random uncertainty from the $\mathrm{S} / \mathrm{N}$ of optically thin lines. This asymmetry is apparent in many of the uncertainties for Si II and Fe II in Table 4. The effect is less pronounced for Ni II where typically all transitions have $\tau<1$.

Good agreement between multiple transitions of the same ion additionally confirms that the analysis does not suffer from large systematic errors. Optically thin lines such as the Ni II transitions are highly sensitive to the continuum level, and their mutual consistency verifies that the continuum estimates are reliable.

\subsubsection{Neutral Hydrogen}

$\mathrm{H} \mathrm{I}$ is the dominant low ion and represents the essential reference scale for absolute abundance measurements. Three galaxies in our sample are at sufficiently high redshift $(z>2.5)$ to determine $\mathrm{H}$ I column densities from the Ly $\alpha$ transition in Keck/ESI spectra. Additionally, CSWA 128 has a high-quality MMT spectrum reaching the necessary blue wavelengths (described by Stark et al. 2013). In these cases, we fit the Ly $\alpha$ absorption with a Voigt profile. We fix the centroid and Doppler parameter to the centroid and standard deviation of the low-ionization covering fraction profiles shown in Figure 6, allowing the H I column density and covering fraction to vary. Wavelengths affected by Ly $\alpha$ emission or by absorption features (e.g., Si III $\lambda 1206$ or the Ly $\alpha$ forest) are excluded from the fits. The resulting best-fit profiles are shown in Figure 7. As with the metal ion column densities, we define an average line of sight,

$$
N_{\text {tot }}=N f_{c},
$$


Table 3

Column Densities of Individual Transitions

\begin{tabular}{|c|c|c|c|c|c|c|c|c|c|}
\hline $\begin{array}{l}\text { Transition } \\
\Delta v\left(\mathrm{~km} \mathrm{~s}^{-1}\right)\end{array}$ & $\begin{array}{l}\text { CSWA } 141 \\
-400 \text { to }-150\end{array}$ & $\begin{array}{l}\text { CSWA } 103 \\
-500 \text { to } 0\end{array}$ & $\begin{array}{l}\text { CSWA } 19 \\
-250 \text { to } 0\end{array}$ & $\begin{array}{l}\text { CSWA } 40 \\
-250 \text { to } 50\end{array}$ & $\begin{array}{c}\text { CSWA } 2 \\
-200 \text { to } 300\end{array}$ & $\begin{array}{l}\text { CSWA } 128 \\
-300 \text { to } 100\end{array}$ & $\begin{array}{l}\text { CSWA } 164 \\
-400 \text { to } 150\end{array}$ & $\begin{array}{l}\text { CSWA } 39 \\
-300 \text { to } 0\end{array}$ & $\begin{array}{l}\text { CSWA } 38 \\
-400 \text { to } 0\end{array}$ \\
\hline H I $1216^{\mathrm{a}}$ & $\ldots$ & $\ldots$ & $\cdots$ & $\ldots$ & $\ldots$ & $21.22_{-0.25}^{+0.23}$ & $20.28_{-0.35}^{+0.26}$ & $20.13_{-0.60}^{+0.71}$ & $20.57_{-0.17}^{+0.16}$ \\
\hline O I $1302^{b}$ & $\ldots$ & $\ldots$ & $\ldots$ & $>20$ & $>51$ & $>30$ & $>28$ & $>10$ & $>34$ \\
\hline $\mathrm{Mg}$ II $2803^{\mathrm{b}}$ & $>0.87$ & $\cdots$ & $\cdots$ & $\cdots$ & $\cdots$ & $\cdots$ & $\cdots$ & $\cdots$ & $\cdots$ \\
\hline Al II 1670 & $\cdots$ & $\cdots$ & $0.12_{-0.02}^{+0.03}$ & $\ldots$ & $\ldots$ & $\cdots$ & $0.15_{-0.02}^{+0.05}$ & $\ldots$ & $\ldots$ \\
\hline $\mathrm{Al}$ II $1670^{\mathrm{b}}$ & $\cdots$ & $>0.69$ & $\cdots$ & $>0.44$ & $>1.10$ & $>0.65$ & $\ldots$ & $>0.22$ & $>0.75$ \\
\hline Cr II 2066 & $2.84_{-1.39}^{+-\mathrm{NaN}}$ & $0.79_{-0.55}^{+1.20}$ & $-0.28_{-0.23}^{+0.46}$ & $\cdots$ & $\cdots$ & $0.05_{-0.37}^{+0.53}$ & $\cdots$ & $-0.60_{-0.27}^{+0.60}$ & $0.75_{-0.62}^{+1.12}$ \\
\hline Fe II 1608 & $\ldots$ & $13.53_{-1.69}^{+1.89}$ & $0.46_{-0.35}^{+0.94}$ & $8.42_{-1.70}^{+4.34}$ & $13.66_{-2.73}^{+5.91}$ & $12.34_{-1.41}^{+2.57}$ & $-0.12_{-0.43}^{+0.73}$ & $3.17_{-0.96}^{+1.18}$ & $4.18_{-0.91}^{+2.47}$ \\
\hline Fe II 2249 & $\cdots$ & $27.60_{-13.16}^{+22.99}$ & $\cdots$ & $38.11_{-14.54}^{+34.75}$ & $39.21_{-36.86}^{+114.88}$ & $-11.78_{-10.23}^{+15.78}$ & $\cdots$ & $\ldots$ & \\
\hline Ni II 1317 & $\cdots$ & $\cdots$ & $\cdots$ & $\cdots$ & $\cdots$ & $\cdots$ & $1.74_{-0.71}^{+1.14}$ & $0.51_{-0.60}^{+1.31}$ & $1.79_{-0.54}^{+0.84}$ \\
\hline Ni II 1370 & $\ldots$ & $\ldots$ & $-0.28_{-0.40}^{+0.93}$ & $0.07_{-0.93}^{+4.39}$ & $\ldots$ & $2.56_{-1.06}^{+2.83}$ & $-0.67_{-0.39}^{+0.68}$ & $0.10_{-0.42}^{+0.85}$ & $-0.11_{-0.63}^{+1.34}$ \\
\hline Si I 1693 & $\cdots$ & $0.41_{-0.28}^{+0.48}$ & $0.37_{-0.12}^{+0.23}$ & $-0.17_{-0.27}^{+0.90}$ & $-0.82_{-0.64}^{+1.32}$ & $0.10_{-0.17}^{+0.27}$ & $0.08_{-0.18}^{+0.41}$ & $\cdots$ & $0.18_{-0.21}^{+0.34}$ \\
\hline Si I 2515 & $0.52_{-0.21}^{+0.32}$ & $0.25_{-0.14}^{+0.20}$ & $\cdots$ & $\ldots$ & $0.42_{-0.32}^{+0.78}$ & $\ldots$ & $\ldots$ & $\cdots$ & $\cdots$ \\
\hline Fe I 2167 & $-0.02_{-0.21}^{+0.42}$ & $0.13_{-0.18}^{+0.29}$ & $\cdots$ & $\ldots$ & $\ldots$ & $-0.11_{-0.09}^{+0.12}$ & $\ldots$ & $-0.18_{-0.14}^{+0.19}$ & $\ldots$ \\
\hline Fe I 2484 & $-0.06_{-0.04}^{+0.10}$ & $\cdots$ & $\cdots$ & $\cdots$ & $\cdots$ & $\cdots$ & $\cdots$ & $\cdots$ & $\cdots$ \\
\hline Fe I 2523 & $-0.05_{-0.12}^{+0.33}$ & $0.04_{-0.06}^{+0.09}$ & $\ldots$ & $\ldots$ & $\ldots$ & $\ldots$ & $\cdots$ & $\cdots$ & $\ldots$ \\
\hline Al III 1854 & $\ldots$ & $1.10_{-0.12}^{+0.21}$ & $0.58_{-0.08}^{+0.12}$ & $0.33_{-0.11}^{+0.24}$ & $1.23_{-0.25}^{+0.91}$ & $0.93_{-0.11}^{+0.29}$ & $0.34_{-0.06}^{+0.12}$ & $0.33_{-0.06}^{+0.24}$ & $\ldots$ \\
\hline Al III 1862 & $\cdots$ & $1.36_{-0.18}^{+0.37}$ & $0.81_{-0.13}^{+0.20}$ & $1.29_{-0.31}^{+\infty} \mathrm{d}$ & $2.27_{-0.49}^{+\infty}{ }^{d}$ & $1.33_{-0.16}^{+0.38}$ & $0.51_{-0.13}^{+0.17}$ & $0.54_{-0.12}^{+0.13}$ & $1.52_{-0.25}^{+0.62}$ \\
\hline
\end{tabular}

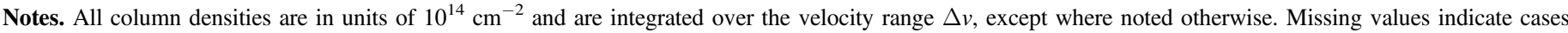
where we are unable to obtain a reliable measurement due to spectral coverage, sky-line residuals, low throughput, or other problems.

${ }^{\mathrm{a}} \mathrm{H}$ I column densities are given as $\log \left(N \times f_{c}\right)$ from Voigt profile fitting, with $N$ in units of $\mathrm{cm}^{-2}$.

${ }^{\mathrm{b}}$ Lower limit corresponding to optical depth $\tau>2$.

c $2 \sigma$ upper limit.

${ }^{\mathrm{d}}$ No formal upper bound.

for $\mathrm{H} \mathrm{I}$ and list the values in Table 3 . We note that $N_{\text {tot }}$ has a lower uncertainty than the best-fit $N(\mathrm{HI})$ due to covariance between $N$ and $f_{c}$.

The column density $N_{\text {tot }}(\mathrm{HI})$ is derived using a different method from that for metal ions and may be subject to different systematic uncertainties. We have assumed that $\mathrm{H} \mathrm{I}$ is coincident with low-ionization metals in fixing the kinematics of the Ly $\alpha$ Voigt profile fits. Best-fit covering fractions provide a sanity check: in all cases, the $\mathrm{H}$ I covering fractions are in agreement with $f_{c}$ derived from low-ionization metals, consistent with a cospatial distribution of $\mathrm{H} \mathrm{I}$ and lowionization metal absorption. For CSWA 39, the uncertainty is large $\left(f_{c}=0.71_{-0.32}^{+0.29}\right.$ for $\left.\mathrm{H} \mathrm{I}\right)$; adopting $f_{c} \simeq 0.4$ as for the low ions would increase $N_{\text {tot }}(\mathrm{HI})$ by $0.35 \mathrm{dex}$, within the uncertainty quoted in Table 3.

\subsection{Neutral Metal Transitions}

Most elements that we consider in this work have first ionization potentials that are below 1 Rydberg, such that both the neutral and first ions may be present in $\mathrm{H}$ I gas. For several elements, we have access to transitions from both the neutral and singly ionized states (notably $\mathrm{Si}, \mathrm{Fe}$, and in the case of CSWA 141 , also $\mathrm{Mg}$, with first ionization potentials $\sim 8 \mathrm{eV}$ or 0.6 Rydberg). In general, these neutral ions are not detected, and their covering fractions are not independently measured. Nonetheless, it is informative to constrain their column densities relative to the first ions. We therefore calculate column densities for several neutral ion transitions assuming that they are cospatial with the low ions (i.e., assuming $f_{c}$ is identical to that derived in Section 3.1 and shown in Figure 6). The results are given in Table 3. In all cases, we find that neutral $\mathrm{Mg}, \mathrm{Si}$, and $\mathrm{Fe}$ are insignificant compared to their singly ionized abundances. The sample median is $N(\mathrm{Si} \mathrm{I}) / N(\mathrm{Si} \mathrm{II})=0.003 \pm 0.003$ with $N(\mathrm{Si}$ I $) / N(\mathrm{Si}$ II $)<0.03$ in all individual cases. We conclude that these neutral species are negligible.

\subsection{Higher Ionization States}

We now turn briefly to the H II gas traced by second and higher metal ions. A key question for this work is whether a substantial fraction of the low-ion column density arises in an ionized (H II) gas phase, in which case relative abundances of low-ion species may require ionization corrections. The most useful probes of higher ionization states in the ESI spectra are Al III $\lambda \lambda 1854,1862$ and Si IV $\lambda \lambda 1393,1402$, which can be compared with the low ions Al II and Si II. Both Al III and $\mathrm{Si}$ IV doublets have a factor of $\simeq 2$ difference in oscillator strength between the two transitions, such that we can jointly fit the column density and covering fraction using the formalism of Equation (3). Covering fractions are valuable for testing whether ionized gas is physically associated with low ions (i.e., having similar $f_{c}(v)$ ), while column densities inform whether 
Table 4

Column Densities from Multiple Transitions

\begin{tabular}{|c|c|c|c|c|c|c|c|c|c|}
\hline $\begin{array}{l}\text { Ion } \\
\Delta v\left(\mathrm{~km} \mathrm{~s}^{-1}\right)\end{array}$ & $\begin{array}{l}\text { CSWA } 141 \\
-400 \text { to }-150\end{array}$ & $\begin{array}{l}\text { CSWA } 103 \\
-500 \text { to } 0\end{array}$ & $\begin{array}{l}\text { CSWA } 19 \\
-250 \text { to } 0\end{array}$ & $\begin{array}{l}\text { CSWA } 40 \\
-250 \text { to } 50\end{array}$ & $\begin{array}{l}\text { CSWA } 2 \\
-200 \text { to } 300\end{array}$ & $\begin{array}{l}\text { CSWA } 128 \\
-300 \text { to } 100\end{array}$ & $\begin{array}{l}\text { CSWA } 164 \\
-400 \text { to } 150\end{array}$ & $\begin{array}{l}\text { CSWA } 39 \\
-300 \text { to } 0\end{array}$ & $\begin{array}{l}\text { CSWA } 38 \\
-400 \text { to } 0\end{array}$ \\
\hline$\overline{\mathrm{Si} \text { II }^{\mathrm{a}}}$ & $17.06_{-28.72}^{+46.39}$ & $79.13_{-20.14}^{+44.67}$ & $6.34_{-1.24}^{+10.17}$ & $56.89_{-11.85}^{+45.84}$ & $59.07_{-10.50}^{+58.36}$ & $50.99_{-8.15}^{+17.21}$ & $10.66_{-2.09}^{+8.73}$ & $22.55_{-8.01}^{+26.52}$ & $33.94_{-6.51}^{+21.45}$ \\
\hline Transitions & 4 & 4 & 3,4 & $2,3,4$ & $1,2,3,4$ & $1,2,3,4$ & $1,2,3,4$ & $2,3,4$ & $1,2,3,4$ \\
\hline $\mathrm{Fe} \mathrm{II}^{\mathrm{b}}$ & $1.49_{-0.22}^{+0.66}$ & $14.70_{-1.17}^{+3.71}$ & $1.07_{-0.20}^{+0.43}$ & $6.29_{-0.93}^{+3.02}$ & $22.76_{-3.17}^{+10.40}$ & $8.65_{-1.12}^{+5.40}$ & $1.50_{-0.24}^{+0.40}$ & $1.92_{-0.90}^{+2.96}$ & $3.50_{-0.41}^{+1.37}$ \\
\hline Transitions & $3,4,5,6,7,8$ & $1,2,3,5,6$ & $1,2,4$ & $1,2,3,4,5,6$ & $1,2,4$ & $1,3,6$ & $1,2,4$ & $1,2,4,5$ & $1,4,6$ \\
\hline $\mathrm{Ni}$ II $^{\mathrm{c}}$ & $\ldots$ & $0.94_{-0.53}^{+1.11}$ & $0.19_{-0.22}^{+0.35}$ & $1.02_{-0.54}^{+0.97}$ & $0.87_{-0.79}^{+3.15}$ & $2.84_{-0.39}^{+0.46}$ & $0.42_{-0.30}^{+0.45}$ & $0.10_{-0.20}^{+0.53}$ & $0.93_{-0.36}^{+0.44}$ \\
\hline Transitions & & 4,5 & $2,3,4,5$ & $1,2,3,4,5,6$ & $1,2,3,4,5$ & $1,2,3,4,5,6$ & $1,2,3,4,5$ & $1,2,3,4,5,6$ & $1,2,3,4,5$ \\
\hline $\mathrm{Al} \mathrm{III}^{\mathrm{d}}$ & $\ldots$ & $1.60_{-0.48}^{+0.68}$ & $0.92_{-0.28}^{+0.47}$ & $\ldots$ & .. & $1.77_{-0.43}^{+0.84}$ & $0.61_{-0.25}^{+0.50}$ & $0.58_{-0.19}^{+0.55}$ & $\ldots$ \\
\hline
\end{tabular}

Notes. All column densities are in units of $10^{14} \mathrm{~cm}^{-2}$ and are integrated over the velocity range $\Delta v$.

${ }^{\mathrm{a}} \mathrm{Si}$ II transitions correspond to $\lambda 1260 \rightarrow 1, \lambda 1304 \rightarrow 2, \lambda 1526 \rightarrow 3, \lambda 1808 \rightarrow 4$.

${ }^{\mathrm{b}}$ Fe II transitions correspond to $\lambda 1608 \rightarrow 1, \lambda 2249 \rightarrow 2, \lambda 2260 \rightarrow 3, \lambda 2344 \rightarrow 4, \lambda 2374 \rightarrow 5, \lambda 2382 \rightarrow 6, \lambda 2586 \rightarrow 7, \lambda 2600 \rightarrow 8$

${ }^{\mathrm{c}} \mathrm{Ni}$ II transitions correspond to $\lambda 1317 \rightarrow 1, \lambda 1370 \rightarrow 2, \lambda 1703 \rightarrow 3, \lambda 1709 \rightarrow 4, \lambda 1741 \rightarrow 5, \lambda 1751 \rightarrow 6$.

d Al III values are based on the $\lambda \lambda 1854,1862$ doublet. 

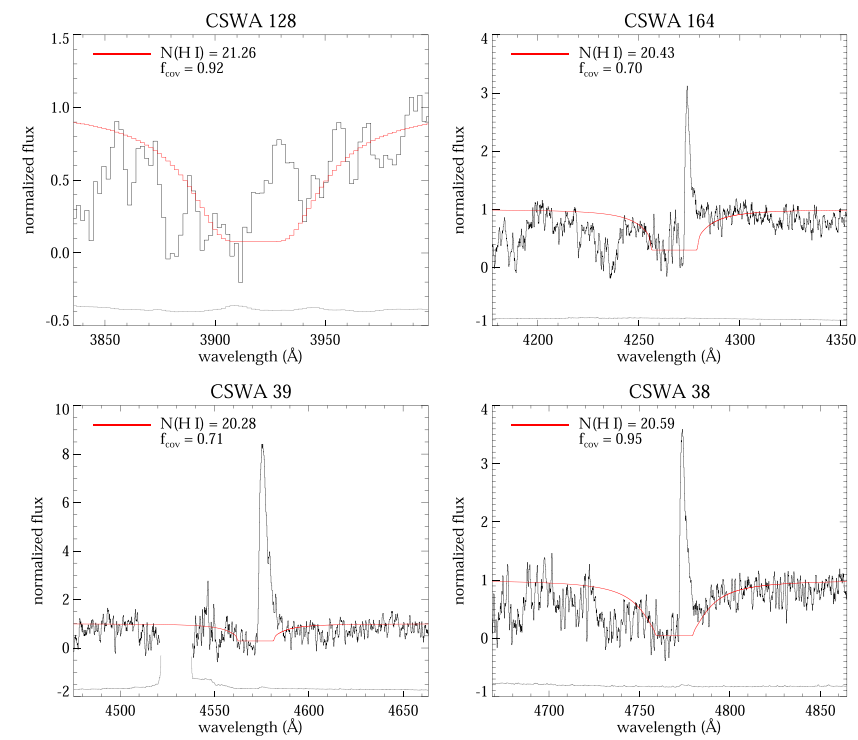

Figure 7. Voigt profile fits to the region around Ly $\alpha$ for galaxies in our sample with suitable spectral coverage. Best-fit parameters corresponding to the red lines are indicated in each panel. $1 \sigma$ error spectra are shown in gray, offset to the bottom of each plot for clarity. The spectrum of CSWA 128 is from MMT/ BCS (Stark et al. 2013) while the rest are from Keck/ESI data presented in this paper.

ionized gas may contribute significantly to the low ions as well as to the total metal content.

The covering fraction profiles of $\mathrm{Al}$ III and Si IV are generally similar to those of low ions. However, we detect systematic differences in $f_{c}$ within the aggregate sample. Si IV covering fractions are on average higher than for the low ions, and this difference is statistically significant within individual velocity channels in some cases (notably for CSWA 19 and CSWA 39). In contrast, Al III covering fractions are systematically smaller than for the low ions. To quantify this difference, we measure column densities $N_{\text {tot }}(\mathrm{Al}$ III) assuming the low-ion $f_{c}$ and list the results in Table $3 . N_{\text {tot }}$ is higher for the weaker transition $\lambda 1862$ in all six cases with reliable measurements (though often at $\lesssim 1 \sigma$ significance). This difference indicates a smaller $f_{c}(\mathrm{Al}$ III) than for the low ions. As a further test, we measure the average ratio of absorption in Al III to the saturated low-ion profiles (i.e., averaged over velocity). This provides a good consistency check with better precision than $N_{\text {tot }}$, and we recover the same values of $N_{\text {tot }}(\lambda 1854) / N_{\text {tot }}(\lambda 1862)$ to better than 0.1 dex. Figure 8 demonstrates that all nine galaxies in the sample have mean ratios indicating that $f_{c}(\mathrm{Al} \mathrm{III})$ is comparable to or smaller than that for the low ions by a factor of $\sim 1-2$. As a result, values of $N_{\text {tot }}$ derived with the low-ion covering fraction are systematically underestimated. The differences may also arise from unresolved saturated components, in which case $N_{\text {tot }}$ is likewise underestimated. We apply an empirical correction by scaling $f_{c}$ (low ion) by a multiplicative factor, such that both $\mathrm{Al}$ III transitions give the same column density. For example, we find $f_{c}(\mathrm{Al} \mathrm{III}) \simeq 0.65 f_{c}$ (low ion) for the case of CSWA 128 , as can be seen in Figure 8 . The resulting values of $N_{\text {tot }}$ are given in Table 4 for all galaxies with reliable constraints.

These differences in covering fraction are explained by separate geometric distributions for the low, intermediate (e.g., Al III), and high (e.g., Si IV) ions. Composite galaxy spectra show that low ions are highly correlated with $\mathrm{H} \mathrm{I}$ Ly $\alpha$ and essentially uncorrelated with the high ions Si IV and C IV

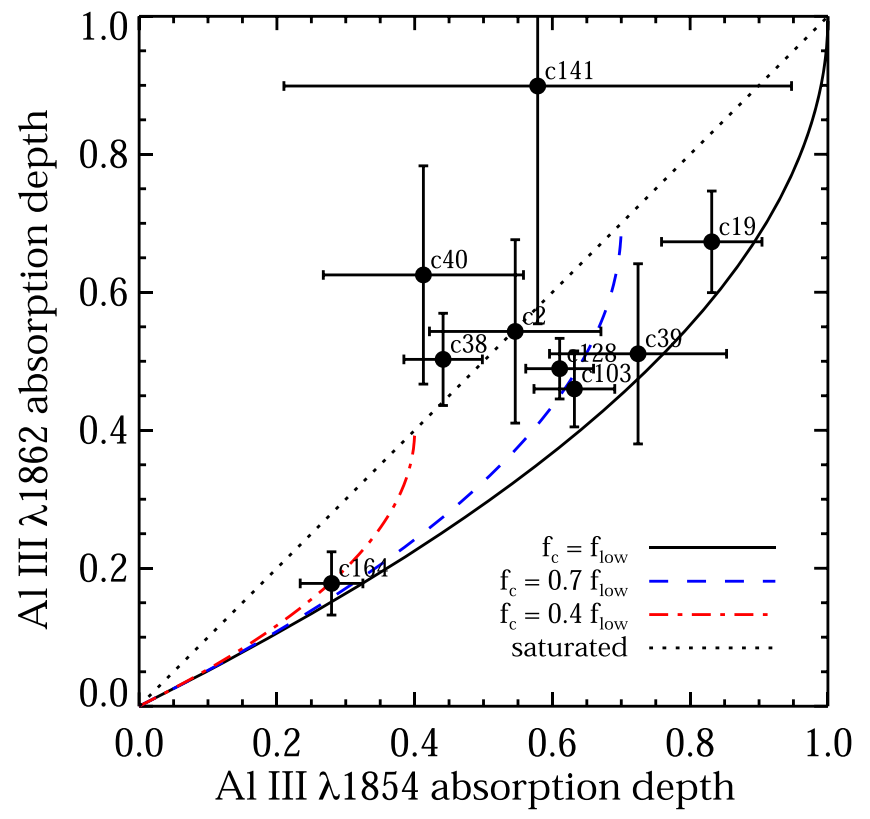

Figure 8. Mean depth of absorption in Al III $\lambda \lambda 1854,1862$ relative to the lowion covering fraction profile $\left(f_{\text {low }}\right)$, calculated over the velocity ranges given in Table 3 and labeled by CSWA catalog number. The solid black line is the locus expected for the case of identical Al III and low-ion covering fractions. All data points lie above this line, indicating that $f_{c}\left(\mathrm{Al}\right.$ III) is smaller than $f_{\text {low }}$. We note that in CSWA 38, Al III $\lambda 1854$ is affected by a sky-line residual, and we consider this measurement to be unreliable. Dashed lines show example loci for different covering fractions and for the case of saturated Al III. The data suggest a range $f_{c}(\mathrm{Al}$ III $) \simeq 0.5-1$ relative to $f_{\text {low }}$.

(Shapley et al. 2003; Jones et al. 2012; Du et al. 2018). Our covering fraction analysis indicates that the lack of correlation is due to different ionization stages occupying distinct spatial regions as opposed to variation in the relative column density of low and high ions. This conclusion is further reinforced by high-resolution spectra of quasar absorption systems, in which high-ion absorption components typically have negligible lowion content (e.g., Wolfe \& Prochaska 2000). On the other hand, Al III absorption is often associated with low ions. We infer that up to $\sim 60 \%$ (i.e., the typical ratio of $\mathrm{Al}$ III to low-ion $f_{c}$ ) of the low-ion absorption in our galaxy sample may be associated with moderately ionized gas traced by Al III. The higher covering fraction of Si IV compared to that of $\mathrm{H}$ I implies a larger volume filling factor for highly ionized gas clouds.

\section{Gas Kinematics}

The kinematics of ultraviolet line profiles in high-redshift galaxies are well known to reflect widespread outflows in addition to a truly interstellar systemic component (e.g., Pettini et al. 2000; Shapley et al. 2003; Steidel et al. 2010; Jones et al. 2012; Law et al. 2012). The vast majority of these studies rely predominantly on the strongest interstellar features, which are typically saturated and therefore trace the covering fraction (i.e., geometry) of the gas rather than the mass. Here we use optically thin lines to quantify the kinematic properties of our sample with respect to the mass distribution traced by column density profiles.

\subsection{The Majority of Detected Gas is Outflowing}

For the purposes of this section, we examine column density profiles derived from individual transitions which are unsaturated, 
Table 5

Gas Kinematics

\begin{tabular}{|c|c|c|c|c|c|c|c|c|}
\hline & CSWA 103 & CSWA 19 & CSWA 40 & CSWA 2 & CSWA 128 & CSWA 164 & CSWA 39 & CSWA 38 \\
\hline \multicolumn{9}{|l|}{ Weighted by $f_{c}$} \\
\hline Low-ion $\bar{v}\left(\mathrm{~km} \mathrm{~s}^{-1}\right)$ & $-259 \pm 9$ & $-159 \pm 17$ & $-141 \pm 16$ & $-19 \pm 27$ & $-161 \pm 10$ & $-108 \pm 10$ & $-117 \pm 20$ & $-183 \pm 9$ \\
\hline Low-ion $\sigma_{v}\left(\mathrm{~km} \mathrm{~s}^{-1}\right)$ & $165 \pm 6$ & $162 \pm 8$ & $179 \pm 7$ & $202 \pm 14$ & $170 \pm 5$ & $220 \pm 4$ & $172 \pm 9$ & $173 \pm 4$ \\
\hline \multicolumn{9}{|l|}{ Weighted by $N_{\text {tot }}$} \\
\hline Low-ion $\bar{v}\left(\mathrm{~km} \mathrm{~s}^{-1}\right)$ & $-212 \pm 21$ & $-143 \pm 32$ & $-156 \pm 45$ & $-32 \pm 54$ & $-133 \pm 25$ & $-42 \pm 27$ & $-115 \pm 42$ & $-260 \pm 37$ \\
\hline Low-ion $\sigma_{v}\left(\mathrm{~km} \mathrm{~s}^{-1}\right)$ & $149 \pm 5$ & $134 \pm 10$ & $96 \pm 17$ & $93 \pm 39$ & $117 \pm 10$ & $137 \pm 13$ & $32 \pm 17$ & $105 \pm 16$ \\
\hline Transitions ${ }^{\mathrm{a}}$ & $1,2,4$ & $2,3,4$ & 1,2 & 1,2 & $1,2,5$ & 3,6 & 1,2 & $1,2,3$ \\
\hline $\mathrm{Al}$ III $\bar{v}\left(\mathrm{~km} \mathrm{~s}^{-1}\right)$ & $-209 \pm 33$ & $-106 \pm 20$ & $-224 \pm 116$ & $52 \pm 73$ & $-113 \pm 27$ & $-117 \pm 59$ & $-160 \pm 37$ & $-171 \pm 55$ \\
\hline $\mathrm{Al} \mathrm{III} \sigma_{v}\left(\mathrm{~km} \mathrm{~s}^{-1}\right)$ & $133 \pm 8$ & $128 \pm 8$ & $113 \pm 30$ & $124 \pm 36$ & $144 \pm 10$ & $170 \pm 22$ & $89 \pm 22$ & $134 \pm 16$ \\
\hline
\end{tabular}

Note.

${ }^{\mathrm{a}}$ Low-ion transitions correspond to $\mathrm{Si}$ II $\lambda 1808 \rightarrow 1$, Fe II $\lambda 1608 \rightarrow 2$, Fe II $\lambda 2344 \rightarrow 3$, Fe II $\lambda 2374 \rightarrow 4$, Ni II $\lambda 1741 \rightarrow 5$, Al II $\lambda 1670 \rightarrow 6$.

well detected, and free of contamination. Two to three of the best such transitions in each spectrum are averaged to construct a mean low-ion column density profile. Table 5 summarizes the mean velocity of the column density profile $\bar{v}$, the dispersion $\sigma_{v}$ (corrected for spectral resolution), and the transitions used. CSWA 141 is excluded from this analysis due to the limited velocity range of our measurements.

The negative mean velocities in Table 5 indicate a net outflow of the gas. Here we attempt to separate the absorption into outflowing and systemic (at rest with respect to the stars) components in order to determine their relative dominance. We define the net outflowing column density as

$$
N_{\text {out }}=\int_{-\infty}^{0} N(v) d v-\int_{0}^{\infty} N(v) d v,
$$

i.e., the difference between the integrated column density below and above the systemic velocity. In practice, we integrate over the velocity range shown in Figure 6, again excluding CSWA 141 due to the limited velocity coverage. $N_{\text {out }}$ comprises a total fraction $f_{\text {out }}$ of the total column density. We find a sample mean $f_{\text {out }}=0.81 \pm 0.19$ for the low-ion column density, indicating that the majority of the detected gas is outflowing. This number strictly applies only to the heavy elements contained in low-ionization gas and not to the molecular nor very hot $\left(T \gtrsim 10^{6} \mathrm{~K}\right)$ phases nor unenriched gas which are not probed by these data. Nonetheless, it is clear that outflows dominate the low-ionization gas phase traced by nearUV absorption. Assuming that the remaining 20\% comprises interstellar gas, this implies that the outflowing mass in lowionization metals is larger by a factor

$$
\frac{M_{\text {out }}}{M_{\mathrm{ISM}}} \approx 4\left(\frac{R_{\mathrm{out}}}{R_{\mathrm{ISM}}}\right)^{2},
$$

where $R_{\text {out }}$ and $R_{\mathrm{ISM}}$ are the mean galactocentric distances of the outflowing and interstellar components. We note that this factor could be very large if the outflowing gas is located far from its origin galaxy.

Finally, we compare results from the column density profiles with an equivalent analysis of the covering fraction from saturated low-ion absorption lines. The covering fraction profiles give a sample mean $f_{\text {out }}=0.53 \pm 0.10$, smaller than but consistent with the column density results. We reiterate that column density is a better tracer of the gas mass than the profiles of saturated transitions.

\subsection{Saturated Line Profiles Overestimate the Bulk Velocity Dispersion}

We now examine whether strong absorption line profiles are representative of the underlying column density distribution. For a direct comparison, we calculate $\bar{v}$ and $\sigma_{v}$ from the low-ion covering fraction profiles shown in Figure 6 (i.e., saturated line profiles) using methods identical to those for the column density. Results from both distributions are given in Table 5 and are compared in Figure 9. The mean velocities from both methods are in good agreement, demonstrating that strong ISM lines accurately trace the average bulk velocity of the gas. However, velocity dispersions are systematically lower for the column density profiles compared to the geometric covering fraction. This is true for every individual galaxy in the sample, with statistical significance ranging from $2 \sigma-7 \sigma$ per galaxy. The median velocity dispersion of covering fraction profiles is $63 \%$ or $75 \mathrm{~km} \mathrm{~s}^{-1}$ higher than that for the column density profiles.

The difference in velocity dispersion between the two profiles arises naturally, since saturated lines trace the full gas velocity range rather than the narrower peaks of column density (or equivalently, optical depth). Mean velocities may also be different, and we expect some scatter between $\bar{v}$ derived from the two profiles, although we find good agreement in general. The uncertainties in Table 5 and Figure 9 indicate a low intrinsic scatter of $\sim 10 \mathrm{~km} \mathrm{~s}^{-1}$. We conclude that kinematic studies based on saturated strong ISM lines accurately recover the mean bulk velocity of the gas as traced by its mass distribution but significantly overestimate the velocity spread by $\sim 60 \%$ for this sample. This leads directly to our finding above that saturated line profiles underestimate the fraction of absorbing material associated with net outflow.

\subsection{Al III Kinematics are Comparable to the Low Ions}

It is useful to compare the kinematics of low ions with $\mathrm{Al}$ III to further check whether a substantial fraction of the low-ion column density may be associated with a moderately ionized $\mathrm{H}$ II phase traced by $\mathrm{Al}$ III. We determine the mean velocity $\bar{v}$ and dispersion $\sigma_{v}$ of the $\mathrm{Al}$ III column density profile for each arc using the same method as that for the low ions. Since Al III covering fractions are poorly constrained, we calculate $N_{\text {tot }}$ (Al III) using the low-ion covering fraction profiles with the caveat that this systematically underestimates $N_{\text {tot }}$ (as discussed in Section 3.3). While this affects the numerical values, any qualitative differences between $\mathrm{Al}$ III and the low ions should be preserved. 

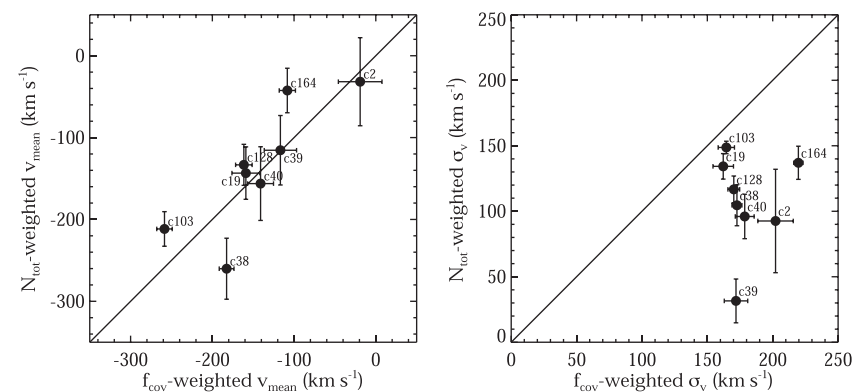

Figure 9. Comparison of the low-ion gas kinematics weighted by the $f_{c}$ and $N_{\text {to }}$ profiles: mean velocity (left) and velocity dispersion (right). The $f_{c}$ weighting reflects the geometric distribution of gas traced by the strongest interstellar features (typically saturated), while the $N_{\text {tot }}$ weighting reflects the column density and hence mass distribution determined from weaker optically thin transitions. Mean bulk velocities are consistent for both the $f_{c}$ and $N_{\text {tot }}$ weighting. Velocity dispersions are systematically $\sim 75 \mathrm{~km} \mathrm{~s}^{-1}$ smaller for the $N_{\text {tot }}$ weighting (median), indicating that the mass is concentrated over a smaller range of velocity than indicated by saturated strong absorption lines.

The results are summarized in Table 5 and compared the with low-ion kinematics in Figure 10. Mean velocities are in good agreement, and their uncertainties are consistent with zero intrinsic scatter between $\bar{v}$ for the low ions and Al III. Velocity dispersions are correlated and higher on average for Al III, with a sample mean difference of $22 \pm 8 \mathrm{~km} \mathrm{~s}^{-1}$ compared to the low ions. This is in qualitative agreement with a study of damped Lyman $\alpha$ (DLA) absorber kinematics by Wolfe \& Prochaska (2000), who found a significant correlation between

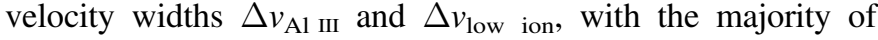
systems having larger $\Delta v_{\mathrm{Al} \text { III. }}$. Given the marginal $\sim 3 \sigma$ significance of the difference in our data, we conclude that the kinematics of Al III and low ions are generally similar with tentative evidence of a broader velocity range for Al III.

\subsection{Velocity Widths Correspond to Extreme DLAs}

The velocity width of low-ion absorption can be directly compared with that of (sub-)DLA systems at similar redshift. Since the H I column densities of our sample are characteristic of DLAs, we expect similar systems tracing galaxy outflows to be present in quasar absorber samples (though they may be rare). Previous studies have quantified the velocity width using the $\Delta V_{90}$ statistic, defined as the velocity range encompassing the 5 th to 95th percentile of the total optical depth (i.e., column density; Prochaska \& Wolfe 1997). Although $\Delta V_{90}$ is not well determined for the galaxies studied here, we can estimate $\Delta V_{90} \approx 3.29 \sigma_{v}$ as appropriate for a Gaussian column density profile. This yields a mean and median $\log \Delta V_{90}\left(\mathrm{~km} \mathrm{~s}^{-1}\right)=2.55$ for the $N_{\text {tot }}$-weighted $\sigma_{v}$. Figure 11 demonstrates that these widths are at the extreme upper end of (sub-)DLA values, well above typical DLAs and comparable to the most metal-rich and dust-depleted systems (with metallicities $\gtrsim 0.1$ solar; Ledoux et al. 2006; Quiret et al. 2016; Ma et al. 2017). Conservatively adopting $\Delta V_{90}>2 \sigma_{v}$ implies typical $\log \Delta V_{90}>2.3$, still at the extreme end of typical DLA values (log $\left.\Delta V_{90} \simeq 1.0-2.5\right)$ and coincident with the most metal-rich systems.

Large velocity widths in the galaxy sample reflect a combination of interstellar gas and kinematically dominant outflows, whereas typical DLAs apparently do not span such a broad range of motion. Furthermore, the galaxy spectra sample only one side of the outflow (with negative apparent velocities) and are missing the positive velocity component, hence we expect that the total gas velocity widths are underestimated for
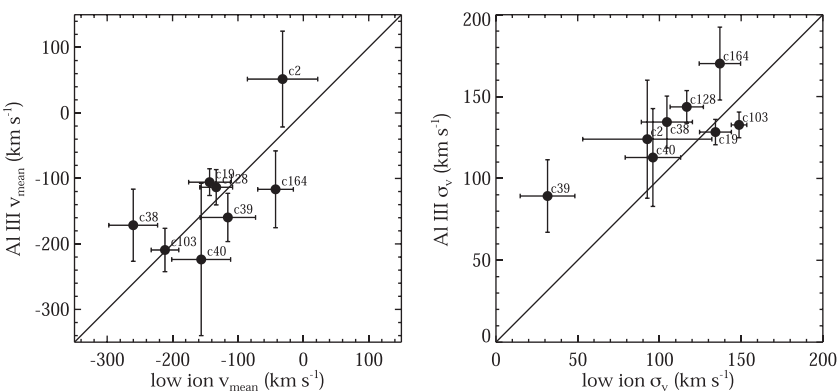

Figure 10. Comparison of $\mathrm{Al}$ III and low-ion gas kinematics: $N_{\text {tot-weighted }}$ mean velocity (left) and velocity dispersion (right). The mean bulk velocities are consistent for both the low ions and Al III. Velocity dispersions for Al III are on average higher than those for low ions, but this difference is not highly significant.

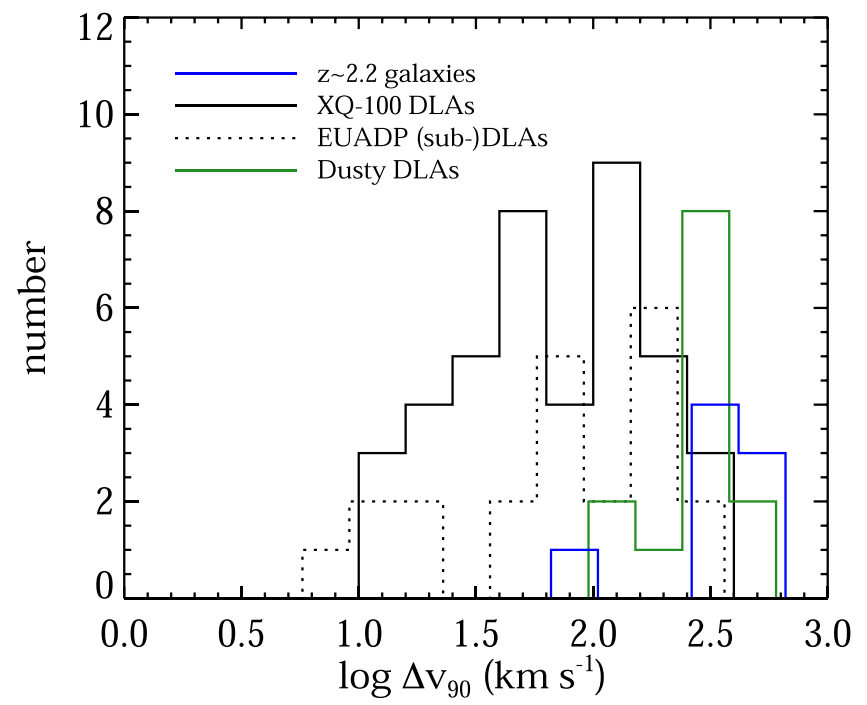

Figure 11. Velocity width distributions of the ESI galaxy sample compared to those of DLA absorption systems in quasar spectra. We show histograms of low-ion $\Delta V_{90}$ from the XQ-100 survey (Berg et al. 2016), EUADP sample (Quiret et al. 2016), and dusty DLAs selected on the basis of $2175 \AA$ dust attenuation features (Ma et al. 2017). Galaxies in our sample have broader velocity distributions than typical DLAs, lying at or beyond the extreme tail of the XQ-100 and EUADP samples. The dusty DLAs have comparable velocity widths. We estimate $\Delta V_{90}=3.29 \sigma_{v}$ for the galaxy sample, noting that redshifted outflowing gas on the far side of the galaxies would further increase $\Delta V_{90}$ by up to 0.3 dex, exacerbating the discrepancy with typical DLAs.

the galaxies. The (sub-)DLA population with similarly broad kinematics may likewise be tracing powerful galactic outflows. If so, the high level of enrichment and depletion seen in these extreme systems likely reflects entrained host galaxy ISM.

\section{Chemical Abundances}

Our column density measurements for multiple elements provide information on chemical abundance patterns, significantly increasing the number of such measurements available for galaxies at $z \gtrsim 2$. Ultimately, we seek to characterize the abundance patterns, chemical enrichment histories, and the likely descendant stellar populations at $z \simeq 0$ based on chemical abundance tagging.

This section focuses on the low ions which provide relative abundances of multiple elements arising from the same (cospatial) gas. The following analysis is based largely on Si II, Fe II, and Ni II column densities, which are reliably 

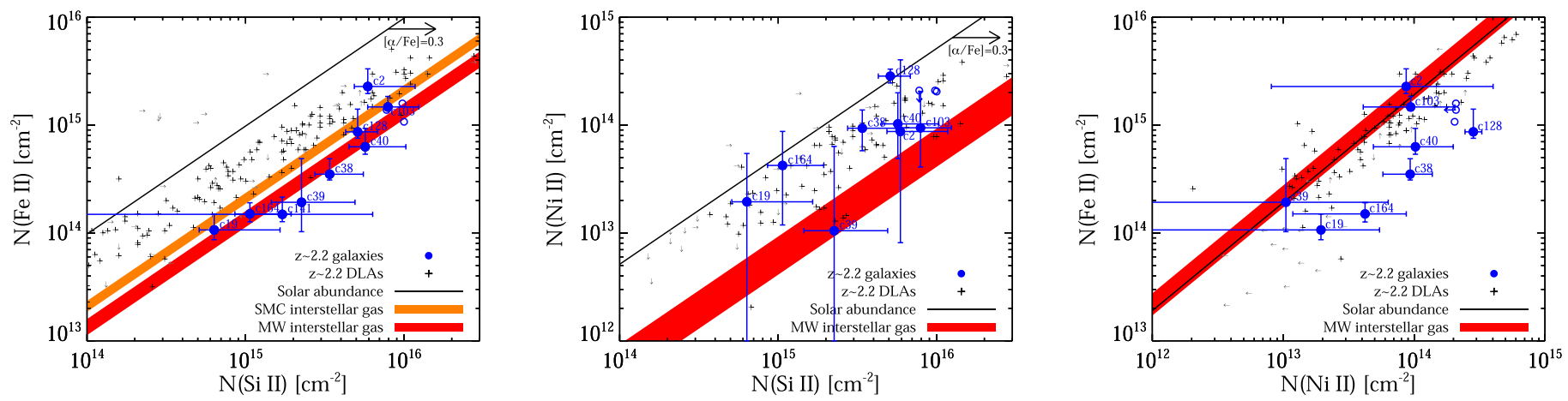

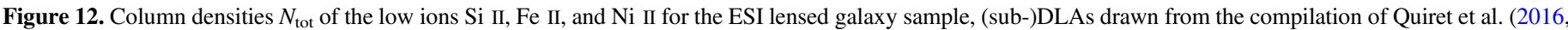

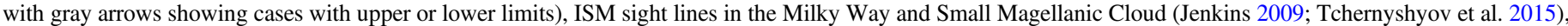

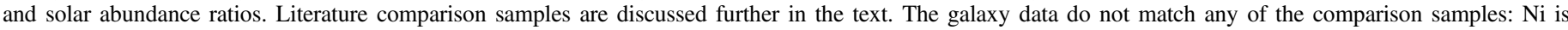

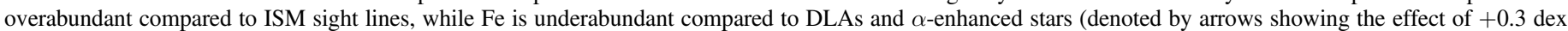

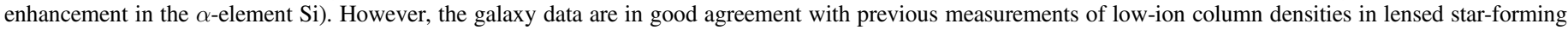

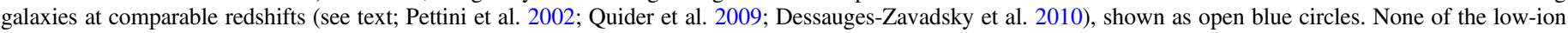
ratios are consistent with solar abundance patterns.

measured for most individual objects. Additional ions in Table 3 provide further constraints, notably $\mathrm{Zn}$ II and Al II. Our approach is as follows. First, we discuss the low-ion abundance patterns and compare them with other classes of objects studied in the literature. Subsequently, we assess possible systematic differences between low-ion and total abundance ratios due to ionization, finite resolution, and dust depletion. We conclude with inferences on the gas-phase abundances as well as the total abundances accounting for depletion.

\subsection{Low-ion Ratios: A Distinct Abundance Pattern}

One of the most striking results of this study is that the lowion column densities reported in Tables 3 and 4 follow a different abundance pattern compared to those seen in other well-characterized classes of absorption line systems. Figure 12 shows the column densities of the low ions Si II, Fe II, and $\mathrm{Ni}$ II along with relevant data from the literature. Low-ion abundance ratios are shown in Figure 13. To proceed with comparisons, we quantify typical column density ratios according to the sample mean ${ }^{5}$ :

1. $\log N(\mathrm{Si}$ II $) / N(\mathrm{Fe}$ II $)=0.85 \pm 0.07 \quad$ (equivalent to $[\mathrm{Si}$ II $/ \mathrm{Fe} \mathrm{II}]=0.84)$.

2. $\log N(\mathrm{Si}$ II $) / N(\mathrm{Ni}$ II $)=1.70 \pm 0.12 \quad([\mathrm{Si}$ II $/ \mathrm{Ni}$ II $]=$ $0.41)$.

3. $\log N(\mathrm{Ni}$ II $) / N(\mathrm{Fe}$ II $)=-0.88 \pm 0.13([\mathrm{Ni}$ II $/ \mathrm{Fe}$ II $]=$ $0.40)$,

where numbers in parentheses are relative to the adopted solar scale. Sample median values are within $1 \sigma$ in all cases. Uncertainties in the sample mean reflect the scatter in values for different objects and are compatible with the statistical measurement uncertainty, but do not include possible systematic errors (e.g., from atomic data or blended absorption features).

Among other ions probed by the spectra, Zn II provides useful constraints despite its low detection significance. The sample mean ratios of $\mathrm{Zn}$ with other elements are as follows:

\footnotetext{
5 CSWA 141 has no measurement for $\mathrm{Ni}$ II and is not included in the latter two ratios. Excluding CSWA 141 altogether would result in a negligible change to $\log N(\mathrm{Si}$ II $) / N(\mathrm{Fe}$ II $)=0.82 \pm 0.07$.
}

$$
\begin{aligned}
& \text { 1. } N(\mathrm{Zn} \mathrm{II}) / N(\mathrm{Si} \text { II })=0.0055 \pm 0.0026 \quad([\mathrm{Zn} \mathrm{II} / \mathrm{Si} \text { II }]= \\
& 0.69) . \\
& \text { 2. } N(\mathrm{Zn} \mathrm{II}) / N(\text { Fe II })=0.030 \pm 0.015 \quad([\mathrm{Zn} \mathrm{II} / \mathrm{Fe} \text { II }]= \\
& \text { 1.42). } \\
& \text { 3. } N(\mathrm{Zn} \mathrm{II}) / N(\mathrm{Ni} \text { II })=0.29 \pm 0.15([\mathrm{Zn} \mathrm{II} / \mathrm{Ni} \text { II }]=1.12) .
\end{aligned}
$$

CSWA 141 and CSWA 39 are excluded from the $\mathrm{Zn}$ analysis due to low precision (e.g., $N(\mathrm{Zn}$ II $) / N(\mathrm{Fe}$ II $)=0.05 \pm 0.06$ for CSWA 141, consistent with the sample mean but with a large uncertainty). These values are given in linear as opposed to logarithmic units in order to account for negative formal column densities. We caution that the $\mathrm{Zn}$ II column densities are derived from the $\lambda 2026$ transition and may be overestimated due to blending with $\mathrm{Mg}$ I. Based on Si I constraints, $\mathrm{Mg}$ I contamination could reduce the derived value of $N(\mathrm{Zn} \mathrm{II}) / N(\mathrm{Si}$ II $)$ by $(7 \pm 9) \times 10^{-4}$ assuming solar ratios of $\mathrm{Mg} \mathrm{I} / \mathrm{Si}$ I. This $\sim 15 \%$ effect is negligible given the uncertainties. Zn II $\lambda 2062$ is not used due to the likelihood of significant blending with $\mathrm{Cr}$ II.

The availability of three low ions-Si II, Fe II, and Ni IIalready provides considerable information about the nature of the absorbing gas for individual galaxies. We compare these ionic abundances with those of several other classes of objects in the remainder of this section: stars in the solar neighborhood, interstellar gas in the Milky Way and Magellanic Clouds, DLA and sub-DLA absorption systems at similar redshift, and previous studies of star-forming galaxies at $z \gtrsim 2$. It is apparent in Figures 12 and 13 that the low-ion abundance patterns in $z \gtrsim 2$ galaxies follow distributions different from these other types of objects. We expand on this conclusion below with a brief discussion of each comparison sample.

Stellar abundances: Figures 12 and 13 show solar abundance ratios as well as the effect of $\alpha$-element enhancement characteristic of rapidly formed stellar populations (e.g., Nomoto et al. 2013). Our low-ion measurements do not follow these stellar abundance patterns. This is perhaps most notable for $\mathrm{Ni}$ and $\mathrm{Fe}$ where $[\mathrm{Ni} \mathrm{II} / \mathrm{Fe}$ II $]=+0.4$, in contrast with the expected stellar $[\mathrm{Ni} / \mathrm{Fe}] \approx 0$. We conclude that the low ions differ from stellar abundance patterns unless the Fe II column densities are systematically underestimated by $\sim 0.4$ dex, which would lead to agreement with the highly $\alpha$-enhanced stellar populations reflecting core-collapse supernova yields (e.g., Bensby et al. 2014). 

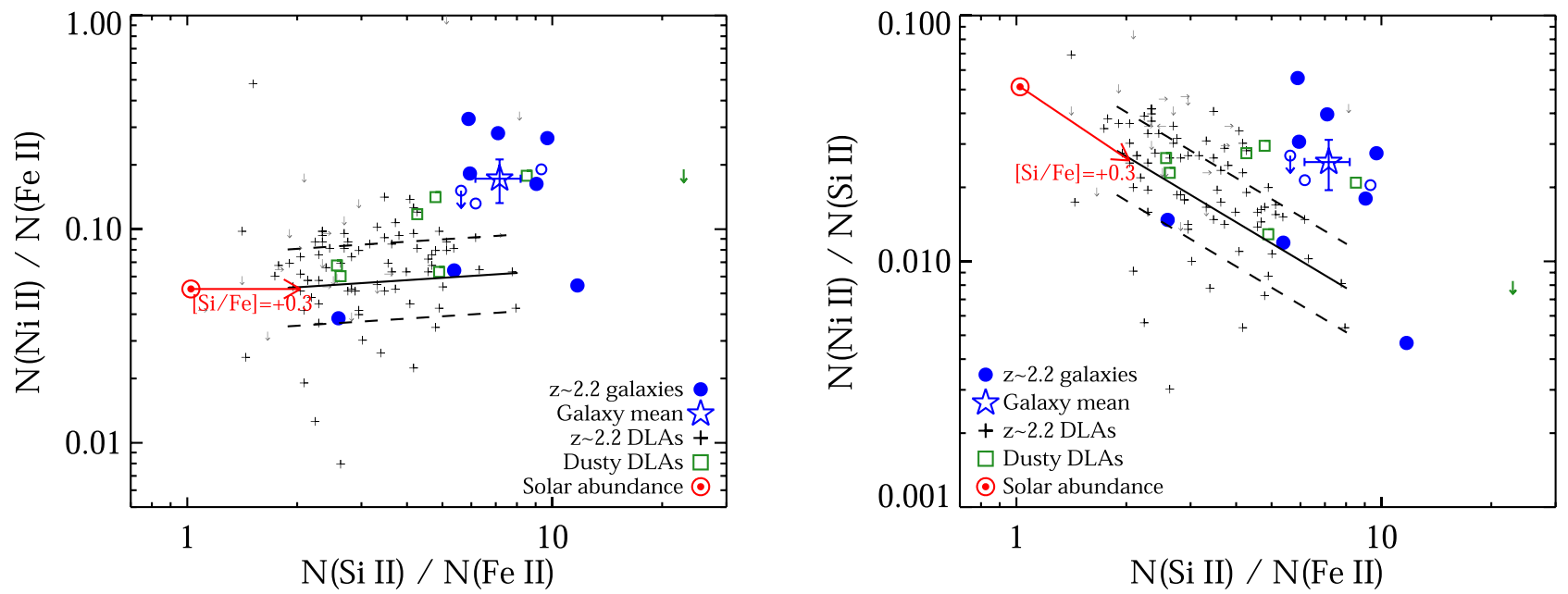

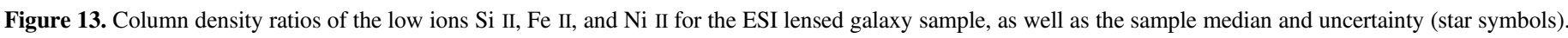

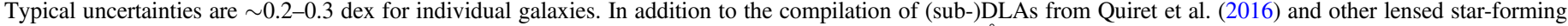

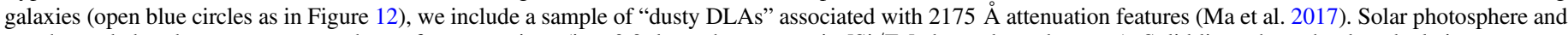

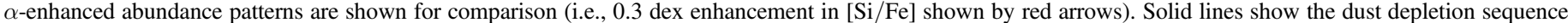
from De Cia et al. (2016) for $[\mathrm{Zn} / \mathrm{Fe}]=0$ to 1 and $[\mathrm{Si} / \mathrm{Fe}]=0.26$, Ni depletions as described in the text, and 0.18 dex scatter in $\mathrm{Ni}$ shown with dashed lines.

Interstellar abundances: We can make a direct comparison with the ISM of nearby galaxies, where abundances are derived using the same UV absorption line analysis. The range of column density ratios found in the Milky Way disk ISM is shown with red shading in Figure 12, corresponding to dust depletion factors $F_{*}=0$ to 1 as defined by Jenkins (2009). Typical ratios of $[\mathrm{Si}$ II/Fe II] in the (less depleted) Small Magellanic Cloud (SMC) are also shown for comparison, corresponding to $F_{*}=0-1$ in Tchernyshyov et al. (2015) or equivalently $F_{*}(\mathrm{SMC}) \simeq-0.3$ to 0.6 in Jenkins \& Wallerstein (2017). The supersolar [Si II/Fe II] gas abundances of our sample are in excellent agreement with the ISM of the Milky Way and Magellanic Clouds, where a large fraction of $\mathrm{Fe}$ is found in solid dust grains. However, $\mathrm{Ni}$ is overabundant by $\sim 0.4$ dex relative to ISM patterns. The ratio of $\mathrm{Ni}$ to $\mathrm{Fe}$ is most constraining, since both elements deplete at similar rates, resulting in near-solar abundances of $[\mathrm{Ni} \mathrm{II} / \mathrm{Fe} \mathrm{II}] \simeq 0$ in the ISM. We conclude that the low ions are different from interstellar abundance patterns unless the Ni II column densities are systematically overestimated.

DLA and sub-DLA abundances: As with interstellar abundances, absorption systems observed along quasar sight lines provide a direct comparison of individual ions. We compare with the compilation of DLA and sub-DLA absorption systems reported in Table B1 of Quiret et al. (2016), which have redshifts and column densities similar to the galaxies presented here. The Quiret et al. (2016) sample has mean and standard deviation $z=2.3 \pm 0.4$ and $\log N_{\mathrm{H} \text { I }}=20.6 \pm 0.5$ (spanning a total range $\log N_{\mathrm{H}}=19-22$ ). Figures 12 and 13 reveal that $\mathrm{Si}$ II/Ni II ratios are typical of (sub-)DLAs. $\mathrm{Ni}$ II/Fe II and $\mathrm{Si}$ II $/ \mathrm{Fe}$ II are typically higher for the galaxies, though consistent with the fringes of the (sub-)DLA distribution. As with stars, we conclude that the low-ion abundances are different from typical quasar absorption systems unless Fe II column densities are systematically underestimated by $\sim 0.4$ dex. This reflects the fact that DLA abundance patterns appear similar to those of old stars in the Milky Way.

Abundances of other galaxies at $z \simeq 2-3$ : This study represents a four-fold increase in the sample of interstellar absorption line abundances available for high-redshift galaxies.
Comparable measurements have previously been obtained for the gravitationally lensed galaxies cB58 at $z=2.73$ (Pettini et al. 2002), CSWA 1 at $z=2.38$ (a.k.a. the "Cosmic Horseshoe"; Quider et al. 2009), and CSWA 21 at $z=2.74$ (a.k.a. the "8 o'clock arc"; Dessauges-Zavadsky et al. 2010). These results are shown as open circles in Figures 12 and 13. Uncertainties are $\sim 0.1$ dex, except for Ni II in CSWA 21, which is an upper limit (denoted by blue arrows). We plot the published values for each source; column densities for CSWA 1 should in principle be multiplied by 0.6 to account for the $60 \%$ covering fraction in our formalism. These previous results lie on the upper end of the column density distribution found in this work. Remarkably, low-ion abundance ratios from previous work agree with our sample mean values to within $\leqslant 0.15$ dex in all cases. We conclude that the low-ion column densities are in excellent agreement with other extant measurements of star-forming galaxies at similar redshift.

\subsubsection{Summary of Abundance Comparisons}

The low-ion column density ratios are inconsistent with abundances of stars, local galaxy ISM, and typical quasar absorption systems. Nonetheless, the low-ion ratios are in agreement with previous findings for star-forming galaxies at similar redshifts. Scatter within the galaxy sample is remarkably low: 0.14 dex rms in Si II/Fe II, and presumably even lower considering the measurement uncertainties. Low-ion abundance patterns measured in our sample therefore represent a distinct, and unique, chemical fingerprint of outflowing gas in $z \simeq 2-3$ galaxies.

The nonstellar patterns reinforce conclusions of earlier works that low ions are not representative of the total abundances, due to dust depletion and other possible effects (Pettini et al. 2002; Dessauges-Zavadsky et al. 2010). In the following sections, we assess the total intrinsic (as opposed to ionic) abundance patterns.

\subsection{Corrections to Low-ion Abundance Ratios}

The total abundance patterns of the interstellar medium should correspond to those of newborn stars. The low-ion 


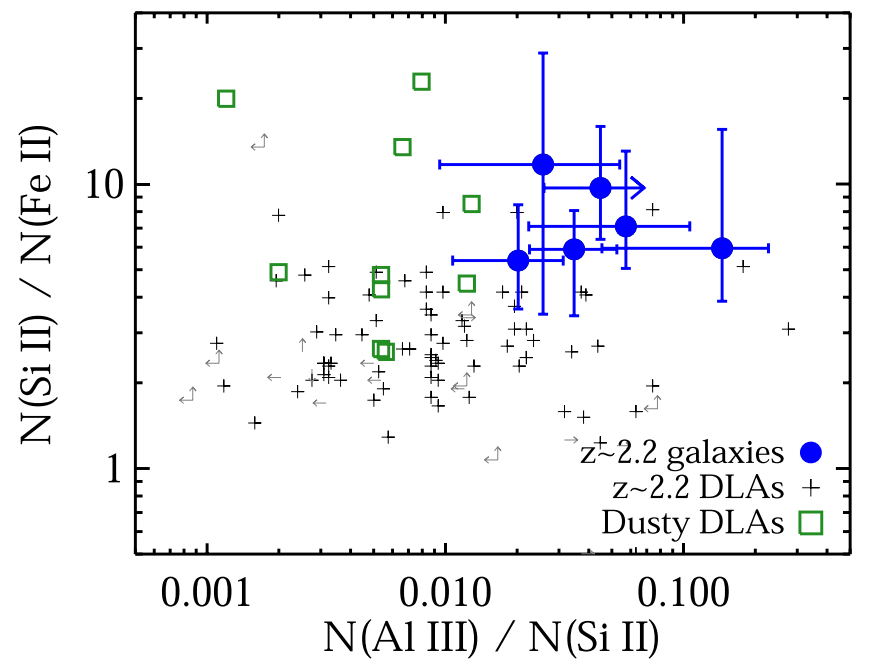

Figure 14. Low-ion column density ratios as a function of ionization state. Symbols are identical to Figure 13. For CSWA 38, N(Al III) is based only on a single transition, and we treat this as a lower limit, appropriate if $f_{c}$ is smaller than for the low ions. The low-ion ratio $\mathrm{Si}$ II/Fe II in these samples shows little or no dependence on the amount of ionized gas traced by Al III. In particular, the high Si II/Fe II seen in galaxy spectra are not observed in DLAs even when comparable amounts of ionized gas are present, indicating that ionization corrections cannot reconcile abundances in the galaxy spectra with the typical DLA population.

abundance patterns in our sample do not match any common stellar population, suggesting that low ions are not representative of the total abundances. In this section, we examine possible causes of this discrepancy. These include ionization corrections, unresolved saturated absorption components, and depletion onto solid dust grains.

\subsubsection{Ionization Corrections}

Ionization corrections are potentially important if a significant fraction of the total low-ion column density is associated with ionized ( $\mathrm{H}$ II) gas. O I is a valuable ionization diagnostic as it is strictly coupled to $\mathrm{H}$ I by charge exchange reactions. Saturated O I $\lambda 1302$ absorption profiles are in good agreement with other low ions in our sample, suggesting a predominantly neutral $\mathrm{H}$ I origin for the low ions. On the other hand, a substantial fraction of the low ions may be associated with moderately ionized gas traced by Al III in our sample (Section 4.3), in which case ionization corrections could be significant. Several groups have used Al III as a diagnostic of ionization corrections in DLA and sub-DLA systems (e.g., Howk \& Sembach 1999; Dessauges-Zavadsky et al. 2003; Lehner et al. 2008; Milutinovic et al. 2010), in conjunction with CLOUDY photoionization modeling (Ferland et al. 2017). We note that the gas probed in galaxy absorption spectra is unlikely to reflect any idealized scenario, and we therefore do not attempt detailed photoionization modeling. Instead, we empirically assess the likely ionization corrections based on the relative behavior of different ions.

Figure 14 shows the abundance of Al III relative to low ions. Ideally, we would use $\mathrm{Al}$ III/Al II to assess ionization corrections, but this ratio is available only in a small number of sources $(<10 \%$ of DLAs in the comparison sample). We therefore use $\mathrm{Al}$ III/Si II as a proxy, noting that this ratio shows relatively little scatter with $\mathrm{Si}$ II/Al II $\simeq 27$ in DLAs and subDLAs (Vladilo et al. 2001; Dessauges-Zavadsky et al. 2003). This ratio implies $\tau \simeq 5-10$ in the $\lambda 1670$ transition for cases

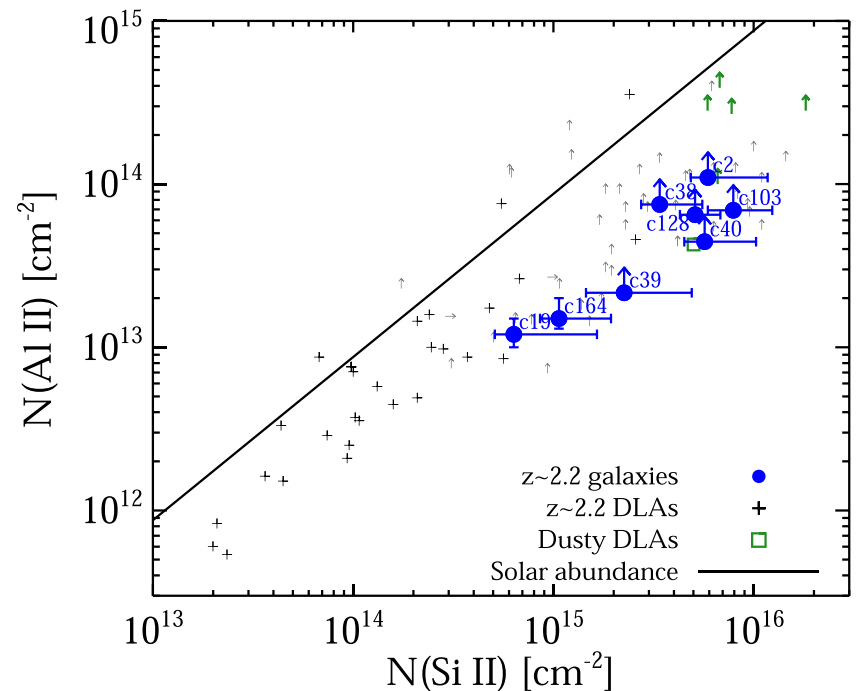

Figure 15. Column densities of Si II and Al II compared with literature samples, similar to Figure 12. In most cases, we derive only lower limits for Al II from the saturated $\lambda 1670$ transition (denoted by arrows); this is also true for the literature data at large column densities. In both galaxies where $N(\mathrm{Al}$ II) is determined directly, we find subsolar [Al II/Si II] comparable to the lowest values seen in DLA absorption systems. In all cases, our lower limits are consistent with subsolar [Al II/Si II]. These results are incompatible with significant stellar ionization.

that we report as saturated in Table 3, consistent with our limits and suggesting $\mathrm{Al}$ III/Al II ratios of order unity. Corresponding ionization corrections from $\mathrm{Al}$ II to total $\mathrm{Al}$ are $\gtrsim 0.2$ dex. While these represent upper limits to the correction required for the low-ionization phase, it is clear that significant amounts of moderately ionized gas are present.

If ionization corrections were responsible for the high $\mathrm{Si}$ II/Fe II and other low-ion ratios observed in the galaxy sample, then we would expect similar values in (sub-)DLAs with large fractions of ionized gas (i.e., high $\mathrm{Al}$ III/Al II and Al III/Si II). Figure 14 shows that this is not the case: even DLAs with similar Al III to low-ion ratios do not match our sample. We find similar null results for both $\mathrm{Ni}$ II/Fe II and $\mathrm{Si}$ II/Fe II, despite their high sensitivity to ionization (with up to $\sim 0.5$ dex corrections possible; e.g., Vladilo et al. 2001). Therefore, while significant amounts of moderately ionized gas are present, ionization corrections do not reconcile the galaxy sample with DLA abundance patterns.

While ionization alone does not explain the difference in abundance patterns between the galaxy sample and DLAs, we nonetheless consider it likely that ionization corrections are important based on the column densities of Al III. We now assess the possible magnitude of such ionization corrections, which depends on the nature of the ionizing sources. The ratio $N(\mathrm{Al}$ II $) / N(\mathrm{Si}$ II) shown in Figure 15 is a useful diagnostic: ionization by a stellar radiation source should result in supersolar [Al II/Si II]. As a fiducial example, we consider the two-region model of Vladilo et al. (2001) with log $N_{\text {H I }}=20.2$ on the low end of our sample. This case results in a +0.53 dex increase in the observed [Al II/Si II], a considerably larger change than that for $\mathrm{Si} / \mathrm{Fe} / \mathrm{Ni}$ low-ion ratios (although possibly overestimated as discussed by Vladilo et al. 2001; Dessauges-Zavadsky et al. 2003). In contrast, the two galaxies with measurements of $N(\mathrm{Al}$ II) show subsolar $[\mathrm{Al}$ II $/ \mathrm{Si} \mathrm{II}]=-0.66$ and -0.79 , highly inconsistent with significant stellar ionization. We suggest that subsolar 
[Al II/Si II] ratios are instead due to a combination of depletion and even-odd nucleosynthetic effects. Furthermore. if the sources with lower limits in $N(\mathrm{Al}$ II) have supersolar ratios, then ionization corrections should be smaller than our estimates above (that is, the true $\mathrm{Al} \mathrm{III/Al} \mathrm{II} \mathrm{would} \mathrm{be} \mathrm{smaller} \mathrm{than}$ estimated from Al III/Si II). Stellar ionization therefore does not explain the low-ion abundance patterns in our sample, indicating that the absorbing gas is sufficiently dense and/or distant from the sites of active star formation. We consider this fiducial case to be a strongly conservative upper limit on the stellar ionization corrections (e.g., $<0.08$ dex correction to [Ni II/Fe II]).

While stellar ionization appears to be insignificant, a hard incident UV spectrum may explain some of the distinct ionic abundance patterns in our sample. Several groups have modeled the effects of ionization by the $z \simeq 2$ extragalactic UV background described by Haardt \& Madau (1996 and subsequent updates). Corrections derived by Howk \& Sembach (1999) for ionization parameters $\log \Gamma \lesssim-3$ are compatible with our data (see also, e.g., Dessauges-Zavadsky et al. 2003; Milutinovic et al. 2010). Such ionization leads to an increase of up to $\lesssim 0.55$ dex for observed [Ni II $/ \mathrm{Fe}$ II]; $\simeq 0$ for $[\mathrm{Ni}$ II $/ \mathrm{Si} \mathrm{II}$; $\lesssim 0.7$ for $[\mathrm{Fe} \mathrm{II} / \mathrm{Zn} \mathrm{II}]$; and $\simeq-0.1$ for $[\mathrm{Al} \mathrm{II} / \mathrm{Si} \mathrm{II}]$, relative to intrinsic elemental abundances. Lower $\Gamma$ values result in smaller corrections. Higher $\Gamma$ would overproduce $\mathrm{Si}$ IV in the galaxies where Si IV is observed while having little effect on the low-ion ratios considered here.

In summary, relatively high Al III column densities suggest that ionization corrections from a hard UV spectrum are likely to be important. However, the lack of correlation between lowion ratios and $\mathrm{Al}$ III suggests that ionization corrections are comparable throughout the sample and approximately independent of the total ionization fraction (i.e., Al III/Al II). We note that the DLA and sub-DLA sample compiled by Quiret et al. (2016) similarly shows little correlation of Si II/Fe II with $\mathrm{Al}$ ionization, and systematically lower values of $\mathrm{Si}$ II/Fe II and $\mathrm{Ni}$ II/Fe II compared to those of the galaxies at fixed Al III/Si II (or Al III/Al II). Therefore, effects other than ionization must contribute to the distinct low-ion ratios observed in the galaxy sample.

\subsubsection{Unresolved Saturated Components}

Due to the moderate spectral resolution and finite size of the target galaxies, the data may be affected by saturated components, which are spectrally or/and spatially unresolved. This would cause a relative increase in the apparent column density of weaker transitions. Based on the optical depths of the transitions used to constrain the column densities, we expect that saturated components would lead to overestimates of [Ni II/Fe II], [Ni II/Si II], and [Si II/Fe II]. We can estimate an upper limit on the magnitude of this effect by assuming a solar intrinsic gas-phase $[\mathrm{Ni} / \mathrm{Fe}]=0$, noting that the true value may be supersolar if DLA abundance patterns are representative. This implies a correction of at most $\lesssim 0.4$ dex to [Ni II $/ \mathrm{Fe} \mathrm{II]}$. By construction, any necessary correction to [Ni II/Fe II] must equal the sum of corrections to [Ni II/Si II] and [Si II/Fe II]. Therefore, we expect $\lesssim 0.4$ dex corrections to each of these ratios due to unresolved saturation. This corresponds to intrinsic gas-phase ion abundances $[\mathrm{Si}$ II $/ \mathrm{Fe}$ II $] \simeq 0.4-0.8$, $[\mathrm{Si} \mathrm{II} / \mathrm{Ni} \mathrm{II}] \simeq 0.4-0.8$, and $[\mathrm{Ni} \mathrm{II} / \mathrm{Fe} \mathrm{II}] \simeq 0-0.4$. Based on studies comparing high- and low-resolution spectra, we expect that corrections should be smaller than this limit, as the absorption spans a broad velocity range, and we have been careful to rely on optically thin lines, which should not be highly biased (e.g., $\tau \lesssim 0.7$; Jorgenson et al. 2013; Cucchiara et al. 2015). In any case, these analyses show that correction for individual transitions should be no more than $0.5 \mathrm{dex}$, with smaller corrections for ion abundance ratios. In summary, unresolved saturation could account for nonsolar [Ni/Fe] lowion abundances only in the extreme case, while $\mathrm{Si}$ remains significantly supersolar relative to the Fe-peak elements in the gas phase.

\subsubsection{Dust Depletion}

Solid dust grains are an important constituent of the ISM metal budget (and CGM; Ménard et al. 2010). Within the Milky Way disk, typically $\gtrsim 90 \%$ of Fe and Ni are depleted into the solid state along with the majority of Si (e.g., Jenkins et al. 1986; Savage \& Sembach 1996), resulting in the nonsolar interstellar gas abundances shown in Figure 12. Smaller depletions are inferred for (sub-)DLA systems $(\simeq 0 \%-90 \%$ of Fe; e.g., De Cia et al. 2016), in some cases comparable to the halo of the Milky Way (Savage \& Sembach 1996). The low-ion column densities reported here enable a similar assessment of dust grain composition in high-redshift galaxies and their associated outflows.

The ratio of [Si II/Ni II] is especially constraining for depletion, since we infer a supersolar gas-phase $[\mathrm{Si} / \mathrm{Ni}] \simeq$ 0.4-0.8 after accounting for the combined effects of ionization $(\lesssim 0.1 \mathrm{dex})$ and saturation $(\lesssim 0.4 \mathrm{dex})$. Such large values can plausibly be explained by moderate depletion (by $\simeq 0.8-1.6 \mathrm{dex}$ in $\mathrm{Fe}$ and $\mathrm{Ni}$ ), extreme $\alpha$-enhancement with Type II supernovae dominating the enrichment, or a combination of these effects. The standard diagnostic [ $\mathrm{Zn}$ II/Fe II] likewise suggests moderate depletion, although this is weak evidence given the $\sim 2 \sigma$ significance of $\mathrm{Zn}$ II absorption. Strong depletion with [Zn/ $\mathrm{Fe}] \gtrsim 2$ is ruled out as inconsistent with the weakness of $\mathrm{Zn}$ II lines; this corresponds to heavily depleted Milky Way sight lines. The subsolar [Al II/Si II] in CSWA 19 and CSWA 164 likewise suggests moderate depletion (e.g., Howk \& Savage 1999), although it could also reflect an odd-even nucleosynthetic effect. Finally, we note that the galaxy low-ion abundance patterns in Figures 13 and 14 most closely resemble the "2DA" quasar absorbers presented by Ma et al. (2017), which are associated with $2175 \AA$ attenuation features, clearly indicating significant dust in these systems. We consider this indirect but compelling evidence that low-ion abundances reflect dust depletion in the galaxy outflows.

A key factor in our interpretation is the dust composition in high-redshift systems. De Cia et al. (2016) have proposed that dust depletion patterns in high-redshift DLAs and local galaxy ISM form a continuous sequence. The DLA-like absorption systems studied here should naturally lie on the same sequence. Since De Cia et al. (2016) do not report results for $\mathrm{Ni}$, we follow their methodology to estimate the Ni depletion sequence using the subset of systems with Ni II, Fe II, Cr II, and Zn II measurements tabulated by Quiret et al. (2016). Cr II is used as a consistency test. We successfully reproduce the depletion pattern

$$
\delta_{X}=A_{X}+B_{X} \times[\mathrm{Zn} / \mathrm{Fe}]
$$

for $\mathrm{Cr}$ with $A_{\mathrm{Cr}}=0.15 \pm 0.04, B_{\mathrm{Cr}}=-1.30 \pm 0.07$, and scatter $\sigma_{\mathrm{Cr}}=0.10$ dex (in excellent agreement with $A_{\mathrm{Cr}}=0.15$, 
$B_{\mathrm{Cr}}=-1.32$, and $\sigma_{\mathrm{Cr}}=0.10$ given by De Cia et al. 2016). The resulting $\mathrm{Ni}$ depletion sequence is characterized by $A_{\mathrm{Ni}}=-0.03 \pm 0.06$, slope $B_{\mathrm{Ni}}=-1.19 \pm 0.11$, and relatively large scatter $\sigma_{\mathrm{Ni}}=0.18$. The resulting depletion sequences are shown in Figure 13 for the range of (depleted) gas-phase $[\mathrm{Zn} / \mathrm{Fe}]=0$ to 1 , with $[\mathrm{Si} / \mathrm{Fe}]=0.26$ as assumed for low-metallicity DLAs by De Cia et al. (2016). The resulting depletion sequence is unable to explain low-ion column densities in the galaxy sample unless $\mathrm{Ni}$ II has been overestimated by $\sim 0.5 \mathrm{dex}$, echoing the conclusions from Section 5.1 regarding Milky Way ISM. Notably, Figure 13 shows that this depletion sequence is unable to explain several of the DLAs from Ma et al. (2017), which are known to be associated with large dust column densities. As with the galaxies, these dusty DLAs are on average overabundant in $\mathrm{Ni}$ II relative to the depletion pattern. This can be explained by additional ionization or saturation effects, combined with the depletion of $\sim 0.6$ dex in Fe and Ni. Alternatively, the dust composition in these systems may be $\mathrm{Ni}$ poor compared to the Milky Way ISM.

In summary, the low-ion column densities show evidence of dust depletion, but depletion patterns seen in the Milky Way and inferred for typical DLAs at $z \simeq 2$ cannot fully reproduce the observations. DLA-like or Milky Way halo-like dust with Fe depletions of $\sim 0.6$ dex can provide a consistent picture in combination with ionization corrections. Another possibility is that the dust composition is fundamentally different from these systems, leading to enhanced gas-phase $\mathrm{Ni}$ abundances similar to those reported for dusty DLAs (Ma et al. 2017). This would indicate a different origin or processing for the dust in highredshift galaxy outflows compared to normal ISM grain growth processes. This would imply a greater depletion.

\subsection{Intrinsic Abundance Ratios}

In this section, we assess the intrinsic heavy element abundance ratios and evidence for departures from the solar pattern. In particular, the $\alpha$-element $\mathrm{Si}$ may be enhanced or decreased relative to $\mathrm{Fe}$ and $\mathrm{Ni}$, depending on the history of star formation and metal mixing. The target galaxies have high specific SFRs such that core-collapse supernovae are expected to dominate the metal production (Mainali et al. 2018, in preparation; Jones et al. 2013; Leethochawalit et al. 2016), resulting in supersolar $[\alpha / \mathrm{Fe}]$. This is reflected in the abundance patterns of metal-poor DLAs and Milky Way stars, which reach $[\mathrm{Si} / \mathrm{Fe}]=0.3-0.4$ (e.g., Becker et al. 2012; Bensby et al. 2014). The observational effect of such an $\alpha$-enhancement is shown in Figures 12 and 13. Zn and Ni may also be moderately enhanced by up to $\sim 0.1 \mathrm{dex}$, but this is a small effect given the observational uncertainties.

It is clear from Figure 13 that $[\mathrm{Si} / \mathrm{Fe}]$ enhancement is highly degenerate with depletion. This arises from the similar depletion of $\mathrm{Ni}$ and $\mathrm{Fe}$ in addition to their common nucleosynthetic pathway, whereas $\mathrm{Si}$ is less depleted. The intrinsic gas-phase abundances of $[\mathrm{Si} / \mathrm{Fe}]$ and $[\mathrm{Si} / \mathrm{Ni}]$, which are constrained to be $\simeq 0.4-0.8$ considering ionization and saturation effects, can readily be explained by a combination of depletion and $\alpha$ enhancement. However, additional elements are needed to distinguish these effects. In this regard, $[\mathrm{Al} / \mathrm{Si}]$ is a useful diagnostic of chemical maturity, as the odd-even nucleosynthetic signature is correlated with $\alpha$-enhancement. We infer a galaxy sample median $[\mathrm{Al}$ II $/ \mathrm{Si}$ II $] \geqslant-0.6$ based on $\mathrm{Al}$ II column density measurements and lower limits. We expect the total $[\mathrm{Al} / \mathrm{Si}]$ to be similar to or higher than $[\mathrm{Al}$ II $/ \mathrm{Si} \mathrm{II}]$, accounting for ionization and depletion. For comparison, we consider Milky Way stellar abundance trends from SDSS APOGEE data (Blanton et al. 2017). Based on a running median of abundance ratios as a function of metallicity, we find that $[\mathrm{Al} / \mathrm{Si}] \geqslant-0.6$ implies maximum $\alpha$-enhancement $[\mathrm{Si} / \mathrm{Fe}] \leqslant$ 0.3 and $[\mathrm{Ni} / \mathrm{Fe}] \leqslant 0.1$. The corresponding metallicity limit is $[\mathrm{Fe} / \mathrm{H}] \geqslant-1.7$, although we may expect a higher metallicity scale if the descendants of our galaxy sample are more massive than the Milky Way.

We consider the above result, $[\mathrm{Si} / \mathrm{Fe}] \leqslant 0.3$, to be a stringent limit, provided that metal-poor Milky Way stars are representative of early nucleosynthesis in our galaxy sample. The limit $[\mathrm{Fe} / \mathrm{H}] \geqslant-1.7$ is compatible with our available measurements of $[\mathrm{Fe} \mathrm{II} / \mathrm{H} \mathrm{I}] \simeq-1.4$, which are likely underestimated due to depletion. Nebular abundance measurements suggest $[\mathrm{O} / \mathrm{H}] \geqslant-0.7$ (with CSWA 141 having $[\mathrm{O} / \mathrm{H}] \simeq-0.7$ based on the direct $T_{e}$ method and higher nebular $[\mathrm{O} / \mathrm{H}]$ for other sources: Jones et al. 2013; Stark et al. 2013; Leethochawalit et al. 2016). In general, adopting higher metallicities as a constraint gives abundance ratio limits closer to the solar value (e.g., $[\mathrm{Si} / \mathrm{Fe}] \leqslant 0.2,[\mathrm{Ni} / \mathrm{Fe}] \leqslant 0.06$, and $[\mathrm{Al} / \mathrm{Si}] \geqslant-0.2$ for Milky Way stars with $[\mathrm{O} / \mathrm{H}] \geqslant-0.7)$.

\subsubsection{Joint Constraints on Depletion and Intrinsic Abundances}

Given the degeneracy between dust depletion and intrinsic abundance ratios (in particular $[\alpha / \mathrm{Fe}]$ and the even-odd effect traced by $[\mathrm{Al} / \mathrm{Si}])$, we now quantify their joint constraints. Our approach is to determine which combinations of depletion, abundance patterns, and ionization corrections can match the observed low-ion column densities. We do not include possible effects of saturation, noting that this would decrease the required ionization corrections with little effect on abundances or depletion.

We construct a model as follows. The depletion sequence is taken from De Cia et al. (2016) and combined with our estimate of nickel depletion described in Section 5.2.3. We adopt fiducial ionization corrections corresponding to gas which is (almost) fully ionized by a $z=2$ extragalactic UV background with ionization parameter $\log \Gamma=-3$ (as described by Howk $\&$ Sembach 1999). The corrections are $c([\mathrm{Si}$ II $/ \mathrm{Fe}$ II $])=0.55$, $c([\mathrm{Ni}$ II $/ \mathrm{Fe} \mathrm{II}])=0.55$, and $c([\mathrm{Zn} \mathrm{II} / \mathrm{Fe} \mathrm{II}])=-0.7$. The ionized gas is assumed to constitute a fraction $f_{\text {ion }}$ of the total Fe II column density, with the rest coming from a neutral region where the low ions are proportional to their total gasphase abundances (i.e., with no ionization correction). This two-phase treatment is motivated by the different covering fractions of ionized gas (i.e., Al III) and low ions. The ionized phase is taken to be an extreme case, such that a mix of ionized and neutral phases can reproduce the full plausible range of ionization corrections. For abundance patterns, we treat the intrinsic $[\mathrm{Si} / \mathrm{Fe}]$ as a free parameter and set $[\mathrm{Ni} / \mathrm{Fe}]=$ $0.3 \times[\mathrm{Si} / \mathrm{Fe}]$ to approximate of the mean trend for Milky Way stars. $[\mathrm{Zn} / \mathrm{Fe}]$ is fixed at the solar value; varying this ratio does not affect the results due to the large uncertainty in $\mathrm{Zn}$ II measurements.

The model has three variables: depletion (parameterized by $\delta(\mathrm{Fe})$, the difference between the total and gas-phase $\mathrm{Fe}$ abundance in dex), ionized fraction $f_{\text {ion, }}$, and the value of the intrinsic $[\mathrm{Si} / \mathrm{Fe}]$ abundance. The resulting low-ion ratios are 

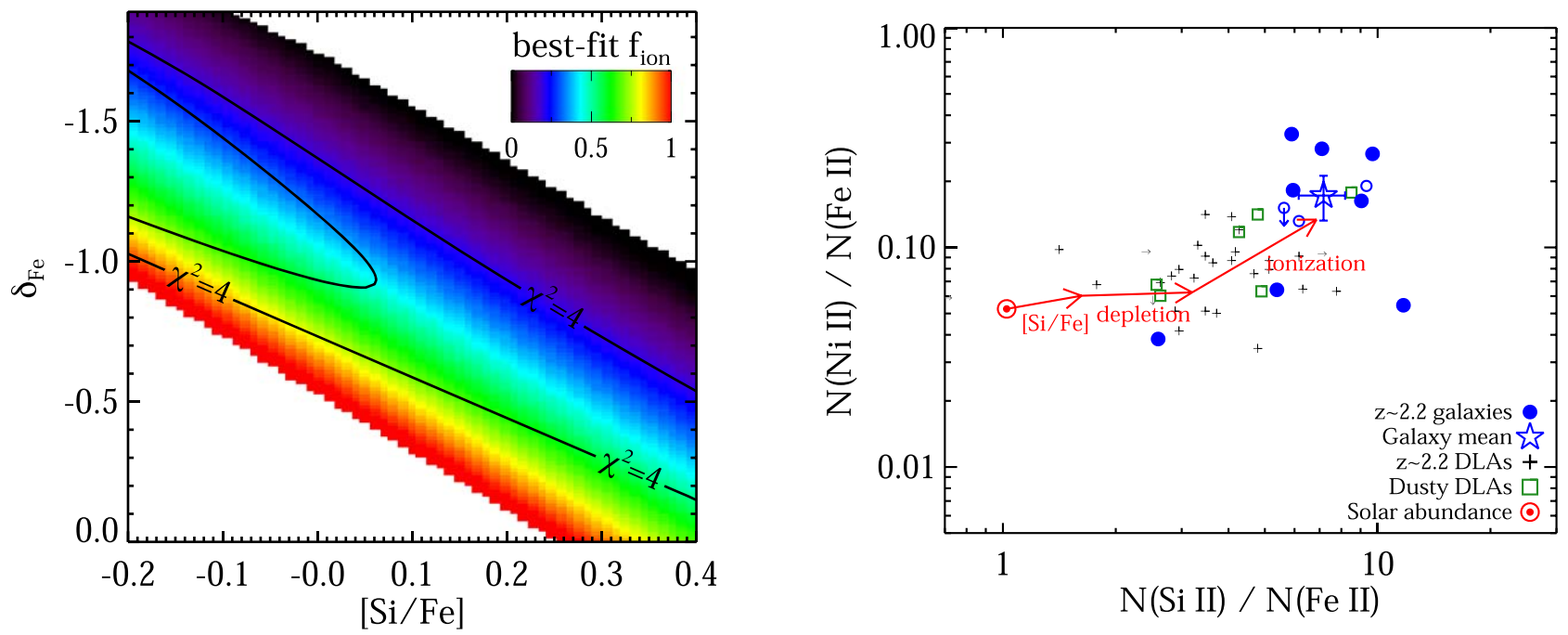

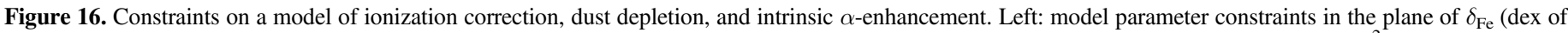

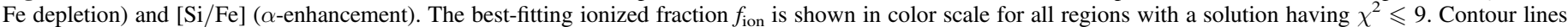

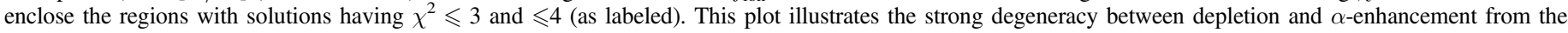

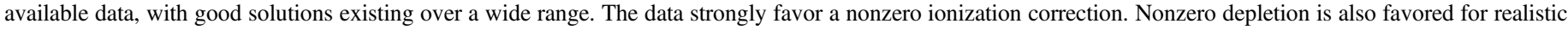

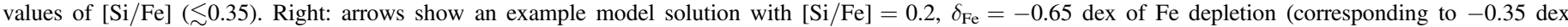

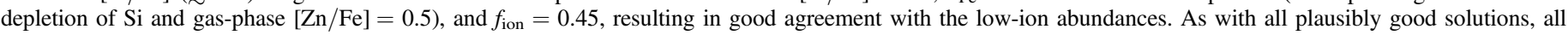

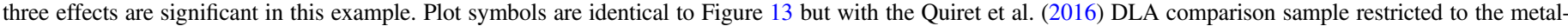

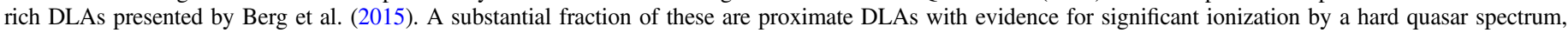
further supporting the importance of ionization corrections.

compared with sample mean values to construct a goodness-offit statistic:

$$
\chi^{2}=\sum_{X} \frac{\left([X / \mathrm{Fe} \mathrm{II}]_{\text {model }}-[X / \mathrm{Fe} \mathrm{II}]_{\mathrm{observed}}\right)^{2}}{\sigma^{2}([X / \mathrm{Fe} \mathrm{II}])},
$$

where $X=\{\mathrm{Si}$ II, Ni II, Zn II $\}$ and $\sigma$ is the observational uncertainty. Fe II is used as the reference normalization scale. Figure 16 shows the results of comparing the model to observed low-ion column densities, with several general features evident. It is clear that this simple model can satisfactorily explain the data with a range of solutions having $\chi^{2}<4$ (i.e., better than $2 \sigma$ agreement with all measurements).

Our aim with this model is to quantify the range of physical conditions that can plausibly explain the low-ion abundances. Figure 16 clearly illustrates the degeneracy between $\alpha$ enhancement, depletion, and ionization corrections. The locus of best-fit solutions can be well described by a linear relation,

$$
\delta_{\mathrm{Fe}}=-0.5+1.8([\mathrm{Si} / \mathrm{Fe}]-0.3),
$$

with a range $\pm 0.3 \mathrm{dex}$ in $\delta_{\mathrm{Fe}}$ corresponding to $\Delta \chi^{2}=1$. The best-fit ionization corrections are described by $f_{\text {ion }} \simeq 0.5$ in the model formalism.

The prescriptions used in our model are not unique, and we have verified that other plausible assumptions can also reproduce the sample mean properties. For example, SMC or Milky Way depletion patterns (Jenkins 2009; Jenkins \& Wallerstein 2017) can be extrapolated to match the data. Different sets of ionization corrections can match the data, subject to the additional constraints discussed in Section 5.2.1. The ability of various models to explain the data underscores the unknown nature of the ionizing spectrum and dust grain compositions. Nonetheless, we can draw some robust general conclusions based on straightforward physical arguments, which are quantitatively supported by the model. In particular:
1. A combination of dust depletion and nonsolar $[\alpha / \mathrm{Fe}]$ is required to explain the data, with larger depletion implying smaller $[\alpha / \mathrm{Fe}]$. This result is driven by the highly supersolar [Si II/Ni II], which is not explained by ionization or saturation (and may even be underestimated if saturation is important). The entire plausible range of $[\mathrm{Si} / \mathrm{Fe}] \simeq-0.1$ to 0.3 can be well fit by the model, in all cases requiring depletion of at least $\sim 0.5 \pm 0.3 \mathrm{dex}$ in $\mathrm{Fe}$.

2. Nonnegligible ionization corrections are required to explain the data. The model is degenerate in the sense that larger ionization corrections imply smaller $[\alpha / \mathrm{Fe}]$ and/or depletion. This result is driven by supersolar [Ni II/Fe II], which is inconsistent with known stellar abundances and depletion patterns. If this ratio is affected by unresolved saturated components, we would infer smaller ionization corrections. Independent of these arguments, Al III column densities suggest that ionization corrections are likely to be important.

We have explained these conclusions without reference to $\mathrm{Zn}$ II, appealing instead to those ions with the most precise column densities. Excluding the $\mathrm{Zn}$ II constraint reduces the overall $\chi^{2}$ but otherwise has little effect on Figure 16. The points above are therefore robust to our inclusion of $\mathrm{Zn} \mathrm{II}$, which serves as a good consistency check.

\section{Discussion}

\subsection{Metallicity and $\alpha / F e$}

One of the goals of this work is to estimate the ISM enrichment of various elements, particularly of the $\alpha$-capture and Fe-peak groups. Total metallicity constraints are available for the four sources with measurements of H I column density from Ly $\alpha$. These sources are representative of the sample in terms of average low-ion abundance ratios (i.e., consistent with 
the mean values in Section 5.1). Their mean gas-phase ion abundance is $\log N(\mathrm{Fe}$ II $) / N(\mathrm{H} \mathrm{I})=-6.06 \pm 0.10$, or

$$
[\mathrm{Fe} \text { II } / \mathrm{H} \mathrm{I}]=-1.56 \pm 0.10
$$

in solar units. We can express the intrinsic metallicity as

$$
\begin{aligned}
{[\mathrm{Fe} / \mathrm{H}] } & \left.=[\mathrm{Fe} \mathrm{II} / \mathrm{H} \mathrm{I}]-\delta_{\mathrm{Fe}}+\text { I.C.(Fe II } / \mathrm{H} \mathrm{I}\right) \\
& \approx[\mathrm{Fe} \text { II } / \mathrm{H} \mathrm{I}]-\delta_{\mathrm{Fe}},
\end{aligned}
$$

where $[\mathrm{Fe} \mathrm{II} / \mathrm{H} \mathrm{I}]$ is the gas-phase value and $[\mathrm{Fe} / \mathrm{H}]$ is the total abundance. The ionization correction term I.C.(Fe II/H I) is expected to be negligible since $[\mathrm{Fe} \mathrm{II} / \mathrm{H} \mathrm{I}] \simeq[\mathrm{Fe} / \mathrm{H}]$ even in highly ionized gas (within $\lesssim 0.1$ dex; e.g., Dessauges-Zavadsky et al. 2003). Likewise, the term for I.C.(Si II/Ni II) is expected to be small, and we can write the intrinsic abundance ratio as

$$
\begin{aligned}
{[\mathrm{Si} / \mathrm{Ni}] } & =[\mathrm{Si} \text { II } / \mathrm{Ni} \text { II }]-\left(\delta_{\mathrm{Si}}-\delta_{\mathrm{Ni}}\right)+\text { I.C. }(\mathrm{Si} \text { II } / \mathrm{Ni} \mathrm{II}) \\
& \approx[\mathrm{Si} \text { II } / \mathrm{Ni} \text { II }]+0.44 \delta_{\mathrm{Fe}} .
\end{aligned}
$$

The relation

$$
\delta_{\mathrm{Si}}-\delta_{\mathrm{Ni}} \approx-0.44 \delta_{\mathrm{Fe}}
$$

is appropriate for the adopted DLA depletion sequence of Section 5.2.3. We note that Equation (14) describes the same locus as the best-fit model of Equation (11).

Equations (13) and (14) together characterize the total abundance $([\mathrm{Fe} / \mathrm{H}])$ and $\alpha$-enhancement $([\mathrm{Si} / \mathrm{Ni}])$ in terms of dust depletion. Substituting our column density measurements into these equations, the typical abundances of the sample are

$$
\begin{aligned}
& {[\mathrm{Fe} / \mathrm{H}] \approx-1.56-\delta_{\mathrm{Fe}} \pm 0.10,} \\
& {[\mathrm{Si} / \mathrm{Ni}] \approx 0.41+0.44 \delta_{\mathrm{Fe}} \pm 0.12}
\end{aligned}
$$

(taking the available [Fe II/H I] measurements as representative; quoted uncertainties are the statistical error in the mean). We have chosen this parameterization to minimize systematic error and covariance: $[\mathrm{Fe} / \mathrm{H}]$ and $[\mathrm{Si} / \mathrm{Ni}]$ rely on independent ion ratios which are relatively insensitive to ionization. The expected corrections are $\lesssim 0.1$ dex, comparable to measurement uncertainties. We illustrate the locus of $[\mathrm{Fe} / \mathrm{H}]$ and $[\mathrm{Si} / \mathrm{Ni}]$ as a function of depletion in Figure 17.

Independent of further analysis, we can characterize the range of abundance patterns allowed by our measurements. It is clear from the above equations and from Figure 17 that negligible depletion would imply metallicities of $\lesssim-1$ in both $[\mathrm{Fe} / \mathrm{H}]$ and $[\alpha / \mathrm{H}]$. This is significantly lower than expected from nebular abundances measured for our sample (as well as expected $[\mathrm{O} / \mathrm{H}] \simeq-0.4$ based on the mass-metallicity relation at $z=2.2$; e.g., Erb et al. 2006; Sanders et al. 2015), unless the outflows are highly diluted by near-pristine gas. At the other extreme, large depletion would imply subsolar $[\alpha / \mathrm{Fe}]$ ratios. The range $\delta_{\mathrm{Fe}}=-1$ to -2 seen in the Milky Way disk corresponds to $[\mathrm{Si} / \mathrm{Ni}]=0$ to -0.5 . Higher depletions are ruled out by gasphase [Zn II/Fe II] abundances. Furthermore, basic chemical evolution arguments suggest that intrinsic $[\alpha / \mathrm{Fe}]$ should be supersolar due to short timescales available for enrichment by Type Ia supernovae. Typical star formation timescales sSFR ${ }^{-1}$ and the $\sim 3 \mathrm{Gyr}$ age of the universe both suggest $[\alpha / \mathrm{Fe}] \gtrsim 0.1$ (e.g., Thomas et al. 2005), and likely higher based on the sSFR. These considerations therefore suggest intermediate depletions with the most plausible range being $\delta_{\mathrm{Fe}} \simeq-0.6 \pm 0.3$. This gives sample mean abundance patterns ranging from

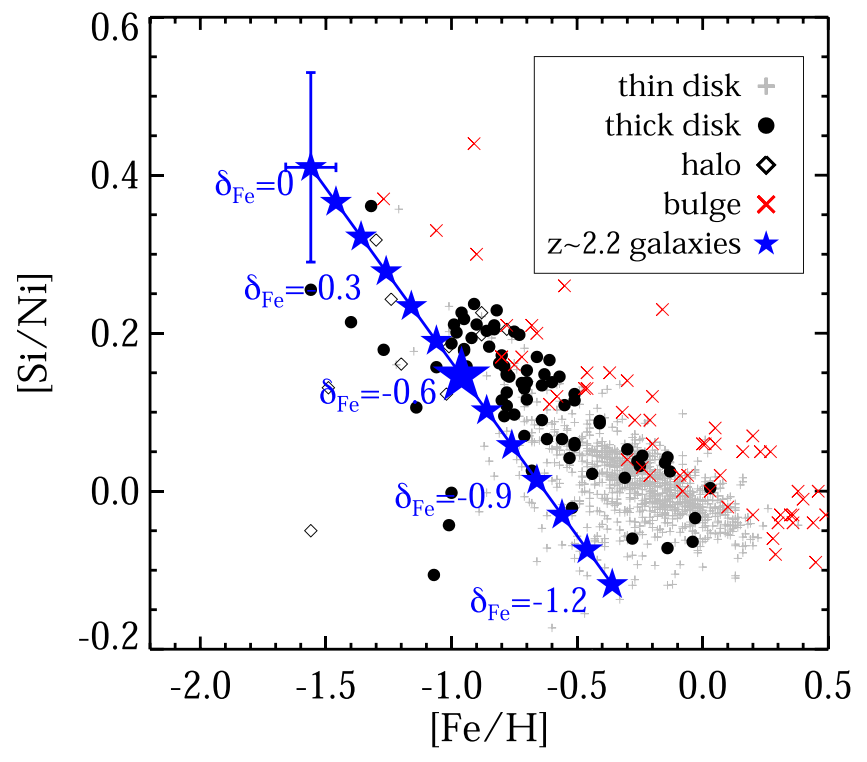

Figure 17. Comparison of Milky Way stellar abundance patterns with interstellar abundances in the $z \simeq 2.2$ galaxy sample (shown for a range of $\mathrm{Fe}$ depletion $\delta_{\mathrm{Fe}}$, with a larger symbol for the fiducial $\delta_{\mathrm{Fe}}=-0.6$ ). Galaxy abundances correspond to Equation (16), with $1 \sigma$ statistical error bars shown on the uppermost point. Stellar data are from Adibekyan et al. (2012) for the Milky Way thin disk, thick disk, and halo, and from Bensby et al. (2013) for the Milky Way bulge.

$[\mathrm{Fe} / \mathrm{H}] \simeq-1.3$ to -0.7 and $[\mathrm{Si} / \mathrm{Ni}] \simeq 0.3$ to 0 , with statistical uncertainties of order 0.1 dex.

\subsection{Comparison to Milky Way Stellar Abundances}

The abundance patterns of the galaxy sample are directly compared with the those of the Milky Way stellar population in Figure 17. The track of abundance patterns for different depletions closely follows that of the thick disk. Thick disk stars are offset $\sim 0.1$ dex higher in $[\mathrm{Si} / \mathrm{Ni}]$ than the galaxy sample mean. Within the uncertainties, mean galaxy abundance patterns may be similar to the thick disk or bulge, or lower by up to $\sim 0.2$ dex in $[\mathrm{Si} / \mathrm{Ni}]$ or equivalently $0.5 \mathrm{dex}$ in $[\mathrm{Fe} / \mathrm{H}]$.

Integral field spectroscopy of the CSWA sample shows that the star-forming gas is characterized by high local velocity dispersions and low ratios of rotation-to-velocity dispersion $(V / \sigma)$, with $\sim 50 \%$ showing signatures of major merger activity (Jones et al. 2013; Leethochawalit et al. 2016). Kinematic information therefore suggests that ongoing star formation is associated with a thick disk or bulge component. If the enrichment timescale is similar to that of the Milky Way thick disk, then the associated $[\alpha / \mathrm{Fe}]$ (i.e., $[\mathrm{Si} / \mathrm{Ni}]$ ) suggests that moderate depletions of $\delta_{\mathrm{Fe}} \simeq-0.4$ to -0.9 are appropriate for the sample. The corresponding gas metallicity is $[\mathrm{Fe} / \mathrm{H}]=$ -1.2 to -0.7 .

We do not necessarily expect agreement between the abundance patterns of the $z \simeq 2.2$ galaxy ISM and of Milky Way stars. However, the average stellar masses of our sample are similar to those expected for Milky Way progenitors (and abundance matching suggests typical $z=0$ descendant masses $\log M_{\star} \simeq 10.8 M_{\odot}$ based on Moster et al. 2013). Given their similar mass scales, the star-forming gas in our sample galaxies should have similar $[\alpha / \mathrm{Fe}]$ compared to Milky Way stars at fixed $[\mathrm{Fe} / \mathrm{H}](\lesssim 0.05$ dex; e.g., Gallazzi et al. 2005; Choi et al. 2014), provided the star formation histories are similar. In contrast, outflows may entrain a significant amount of nearly 
pristine gas, resulting in lower metallicity. The $\lesssim 0.3$ dex agreement in metallicity between outflow-dominated low-ion gas and Milky Way stars, seen in Figure 17, suggests a limited and possibly negligible mass of entrained pristine material ( $\lesssim 50 \%$ of the total outflow mass).

\subsection{Comparison to Nebular Oxygen Abundance}

Oxygen is the most accessible gas-phase metallicity diagnostic for galaxies at $z=2-3$ owing to its strong optical emission lines. A major concern of emission line studies is the uncertainty in absolute abundance scale (e.g., Kewley \& Ellison 2008), although recent work has made progress with direct measurements and calibrations at high redshifts (Jones et al. 2015; Sanders et al. 2016). Emission lines are additionally sensitive to the ionizing spectrum, which depends on stellar $[\mathrm{Fe} / \mathrm{H}]$ and therefore $[\alpha / \mathrm{Fe}]$, which is likely systematically different at high redshifts than in local star-forming galaxies. Independent confirmations of the abundance scale and $[\alpha / \mathrm{Fe}]$ patterns are clearly needed, and our interstellar absorption data provide such complementary measurements.

In several cases, we can make a direct comparison with nebular oxygen abundances in our sample, which suggest typical intrinsic $[\mathrm{O} / \mathrm{H}] \simeq-0.4$ in the young stars and ISM (with uncertainty $\sim 0.2$ dex in the absolute abundance scale; Jones et al. 2013; Leethochawalit et al. 2016). CSWA 141 is an outlier in terms of having strong high-ionization nebular emission lines and low metallicity (as well as low stellar mass and high sSFR), with $[\mathrm{O} / \mathrm{H}]=-0.7$ measured via the direct method from [O III] $\lambda 4363$ (Stark et al. 2013). Overall, our sample is in good agreement with the mass-metallicity relation at these redshifts (e.g., Sanders et al. 2015).

Of the elements probed by our absorption spectra, Si represents the best comparison with nebular $[\mathrm{O} / \mathrm{H}]$. We note that nondetections of $\mathrm{O}$ I $\lambda 1355$ absorption in our spectra provide lower limits $[\mathrm{O} \mathrm{I} / \mathrm{H} \mathrm{I}]=[\mathrm{O} / \mathrm{H}] \gtrsim-1.8$ for those sources with Ly $\alpha$-based measurements of $\mathrm{H} \mathrm{I}$. Although not particularly constraining, these limits are robust to ionization corrections and depletion. The same sources have mean $[\mathrm{Si} \mathrm{II} / \mathrm{H} \mathrm{I}]=-0.7 \pm 0.2$. This is lower than nebular $[\mathrm{O} / \mathrm{H}] \simeq-0.4$ though consistent at the $<2 \sigma$ level, and indeed previous studies have found reasonable agreement between nebular $\alpha$-element abundances and interstellar [Si II/H I] (Pettini et al. 2002; Dessauges-Zavadsky et al. 2010). However, [Si II/H I] is susceptible to large changes from both depletion and ionization (and we suggest that anomalous [Ni II/Fe II] abundances in previous studies may indicate nonnegligible contributions from ionized gas, as in our sample). The effects are opposite in that ionization increases [Si II/H I] while depletion decreases it. Indeed, the example solution shown in Figure 16 has a -0.35 dex depletion of $\mathrm{Si}$ and a 0.28 dex ionization correction to $\mathrm{Si}$ II, such that the effects approximately cancel in this case. In general, the combined systematic effects may be large, however, and we caution that they should be considered carefully. Our best-fit model locus (Equation (11)) corresponds to intrinsic

$$
[\mathrm{Si} / \mathrm{H}]=-0.98-0.44 \delta_{\mathrm{Fe}}
$$

Comparison with the nebular oxygen abundance then gives

$$
\left[\mathrm{Si}_{\mathrm{IS}} / \mathrm{O}_{\mathrm{neb}}\right] \simeq-0.6-0.44 \delta_{\mathrm{Fe}}
$$

explicitly noting that the abundances are from separate interstellar (IS) and nebular (neb) regions. It is clear that Equation (18) is not compatible with solar ratios unless depletion is large or the outflow is diluted with entrained metalpoor gas. We discuss the relative abundances of $\mathrm{Si}$ and $\mathrm{O}$ further in the following section.

\section{4. $\mathrm{O} / \mathrm{Fe}$ and $\mathrm{Si} / \mathrm{O}$}

The degree of $\alpha$-enhancement and $[\mathrm{O} / \mathrm{Fe}]$ in particular has garnered recent interest for its effect on the ionizing stellar spectra of star-forming galaxies at $z \simeq 2-3$. For example, Steidel et al. (2016) found a large value of $[\mathrm{O} / \mathrm{Fe}] \simeq 0.6$ from stacked spectra of $z \simeq 2.4$ galaxies with properties similar to our sample. A direct comparison of $[\mathrm{O} / \mathrm{Fe}]$ with our findings requires an estimate of intrinsic [Si/O] ratios. Both $\mathrm{O}$ and $\mathrm{Si}$ are $\alpha$-elements predominantly generated by core-collapse supernovae, and their ratio is often assumed to be near solar. If we assume solar $[\mathrm{Si} / \mathrm{O}]=0$, a value $[\mathrm{O} / \mathrm{Fe}]=[\mathrm{Si} / \mathrm{Fe}] \simeq 0.6$ is only compatible with our measurements if depletion is negligible. This implies $[\mathrm{Fe} / \mathrm{H}] \simeq-1.6$ and $[\alpha / \mathrm{H}] \simeq-1$, lower than nebular metallicities of both our sample and that of Steidel et al. (2016) by 0.6 dex. If abundances are indeed characterized by solar [Si/O] and high $[\alpha / \mathrm{Fe}]=0.6$, then our measurements imply that the predominantly outflowing ISM seen in absorption must be diluted by a factor of 4 with pristine gas and must have negligible dust content.

Motivated by recent stellar surveys, we consider nonsolar [Si/O] abundance patterns illustrated in Figure 18. Results for the solar ratio with $[\mathrm{O} / \mathrm{Fe}]=[\mathrm{Si} / \mathrm{Fe}]$ are shown as unfilled symbols in Figure 18 and are compatible with SDSS APOGEE measurements for $\alpha$-enhanced stars in the Milky Way (Hayes et al. 2018). In contrast, data from the GALAH survey (Martell et al. 2017; Buder et al. 2018) and from Bensby et al. (2014) indicate that $\alpha$-enhanced stars in the solar neighborhood have $[\mathrm{O} / \mathrm{Fe}] \simeq 2.4 \times[\mathrm{Si} / \mathrm{Fe}]$. As with Figure 17 , our measurements give $\mathrm{Si}$ and $\mathrm{Fe}$ abundances similar to the Milky Way thick disk if depletions are $\delta_{\mathrm{Fe}} \simeq-0.6$. However, the implied $[\mathrm{O} / \mathrm{H}]$ differs by $0.3-0.4$ dex depending on the assumed $[\mathrm{O} / \mathrm{Si}]$ pattern. Which, if either, is correct? As discussed above, $[\mathrm{O} / \mathrm{Si}] \simeq 0$ implies negligible depletion and large mass loading by near-pristine gas. Alternatively, large depletion factors $\delta_{\mathrm{Fe}}<-1$ dex can bring interstellar and nebular $[\mathrm{O} / \mathrm{H}]$ into agreement, but imply subsolar $[\alpha / \mathrm{Fe}]<0$. We note also that if $[\mathrm{O} / \mathrm{Si}]$ is near solar, then our data imply significantly different abundance patterns than found by Steidel et al. (2016). On the other hand, supersolar $[\mathrm{O} / \mathrm{Si}]$ values give a remarkably consistent picture between interstellar measurements, our estimates of nebular abundance $[\mathrm{O} / \mathrm{H}] \simeq-0.4$, and the independent measurements by Steidel et al. (2016), with $\delta_{\mathrm{Fe}}=-0.6$ (Figure 18). We note that Steidel et al. (2016) find nebular $[\mathrm{O} / \mathrm{Si}]=0.63$ from their stacked spectra, implying depletions $\delta_{\mathrm{Si}} \simeq-0.3$ dex (and $\delta_{\mathrm{Fe}} \simeq-0.6$ ) if the intrinsic $[\mathrm{O} / \mathrm{Si}] \simeq 0.3$. Such good agreement in depletion may indicate survival of dust grains as they are accelerated to the outflow velocities we measure in Section 4, or alternatively that dust in outflows and $\mathrm{H}$ II regions undergoes similar processing.

\subsection{Dust-to-gas Ratio}

Abundances and depletion factors can be tested for consistency by considering the mass ratio of dust to gas, or dust to metals. This quantity is also of interest for estimating the total gas masses of distant galaxies, given the relative ease of detecting thermal dust continuum compared to atomic or 

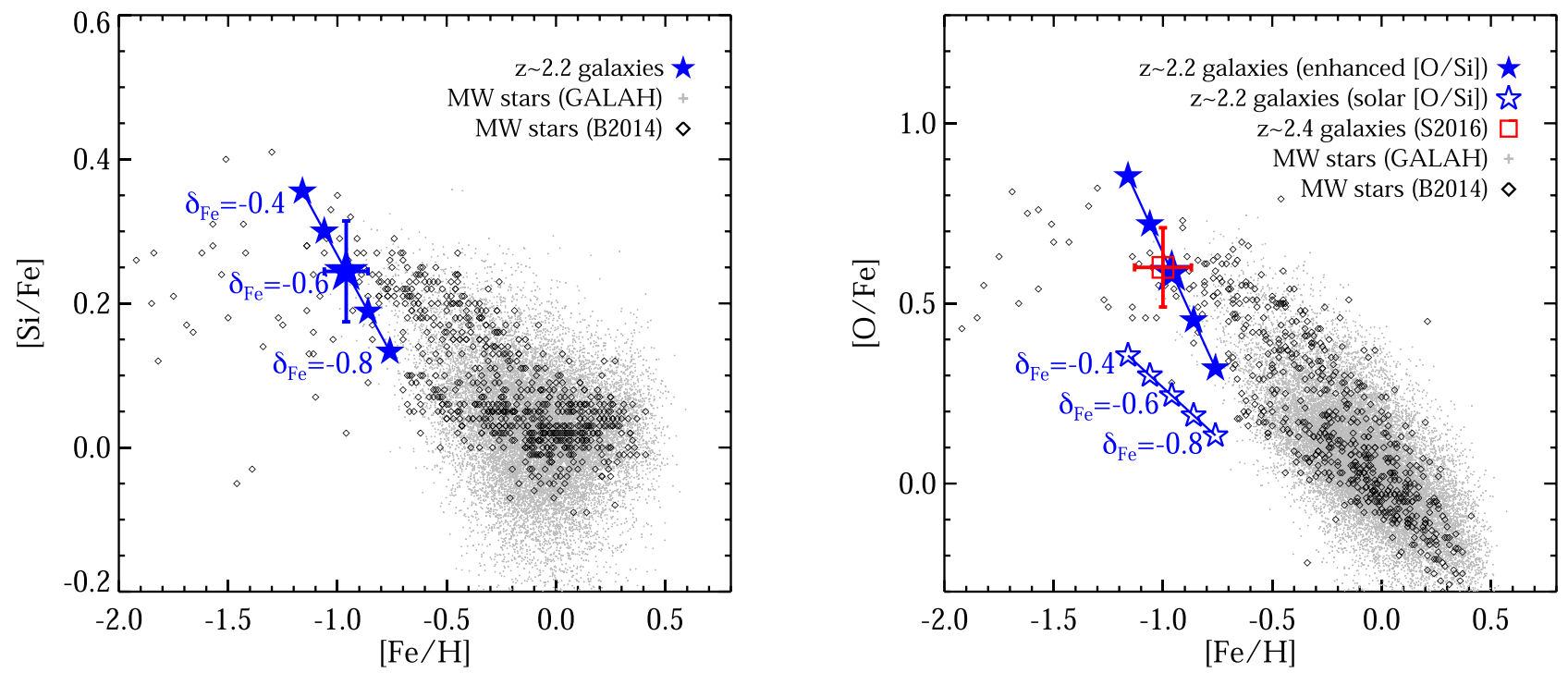

Figure 18. Comparison of Milky Way stellar $[\alpha / \mathrm{Fe}]$ abundance patterns with interstellar abundances in the $z \simeq 2.2$ galaxy sample (blue stars, shown for a range of Fe depletion $\delta_{\mathrm{Fe}}=-0.4$ to -0.8 as labeled). Stellar measurements are from Bensby et al. (2014, B2014) and the GALAH survey (second data release; Buder et al. 2018). Left: $[\mathrm{Si} / \mathrm{Fe}]$ as a function of $[\mathrm{Fe} / \mathrm{H}]$. Depletion of $\delta_{\mathrm{Fe}} \simeq-0.6$ gives abundance patterns consistent with the moderately metal-poor thick disk of the Milky Way. Error bars on the fiducial $\delta_{\mathrm{Fe}}=-0.6$ point show the measurement uncertainty. Right: $[\mathrm{O} / \mathrm{Fe}]$ as a function of $[\mathrm{Fe} / \mathrm{H}]$. We show separate tracks for the $z \simeq 2.2$ sample assuming (1) a solar $[\mathrm{O} / \mathrm{Si}]$ ratio (open stars, consistent with APOGEE abundance patterns), or (2) supersolar $[\mathrm{O} / \mathrm{Si}]$ as described in the text (filled stars, consistent with B2014 and GALAH abundance patterns). The red square represents nebular $[\mathrm{O} / \mathrm{H}]$ and stellar $[\mathrm{Fe} / \mathrm{H}]$ measured by Steidel et al. (2016, S2016) for stacked spectra of galaxies with properties similar to our sample. We find excellent agreement with $\mathrm{S} 2016$ for the case of supersolar $[\mathrm{O} / \mathrm{Si}]$ and depletions $\delta_{\mathrm{Fe}} \simeq-0.6 \pm 0.1$.

molecular gas emission lines (Scoville et al. 2014, 2016). We can express the dust-to-gas mass ratio (DGR) as a function of Fe depletion via

$$
\mathrm{DGR}=\frac{\left(1-10^{\left.\delta_{\mathrm{Fe}}\right)}\right.}{f_{\mathrm{Fe}}} \frac{56}{1.4} \frac{N(\mathrm{Fe})}{N(\mathrm{H})}
$$

where 56 and 1.4 are the approximate mean ion masses (in atomic mass units) for $\mathrm{Fe}$ and for the entire gas phase, respectively. $N(\mathrm{Fe})$ and $N(\mathrm{H})$ refer to the total abundances (i.e., the metallicity). The term $1-10^{\delta_{\mathrm{Fe}}}$ is the fraction of Fe found in solid grains, and $f_{\mathrm{Fe}}$ is the $\mathrm{Fe}$ mass fraction of solid grains. The value of $f_{\mathrm{Fe}}$ is of order $\sim 0.1$ (e.g., 0.22 for the solarmetallicity chondrite composition described by Draine et al. 2007, and we expect lower $f_{\mathrm{Fe}}$ for environments with supersolar $[\alpha / \mathrm{Fe}])$.

For a fiducial abundance $[\mathrm{Fe} / \mathrm{H}]=-1$ and $\delta_{\mathrm{Fe}}=-0.6$ for our sample (e.g., Figure 18), Equation (19) gives

$$
\mathrm{DGR}=10^{-3} \frac{0.1}{f_{\mathrm{Fe}}}
$$

A change of -0.1 dex in $\delta_{\mathrm{Fe}}$ corresponds to +0.13 dex in DGR. This is lower than DGR $\simeq 10^{-2.2}$ found in the Milky Way or in the SINGS galaxies with reliable SCUBA data discussed by Draine et al. (2007), but within the range found by Engelbracht et al. (2008) for galaxies of comparable gas-phase metallicity. DGR exhibits a large scatter at fixed metallicity, which may be due in part to mixing of relatively enriched and pristine material. If so, this may provide further joint constraints on the dust content, total metallicity, and entrainment of pristine gas in outflows. Draine et al. (2007) suggested that $\mathrm{DGR} \approx 0.01 \frac{Z}{Z_{\odot}}$ for unmixed ISM, compatible with our measurements and thereby indicating limited entrainment of pristine gas.
We draw two notable conclusions from the DGR. First, our measurements are consistent with the range seen in local galaxies of similar metallicity, providing a good consistency check and suggesting that outflow composition is similar to the ISM. Second, we infer low DGR compared to the Milky Way ISM, suggesting caution in estimating gas masses based on dust continuum emission. If our measurements are representative of the total ISM, then we expect a factor of $\sim 5$ difference in total gas mass per unit dust emissivity relative to the Milky Way. Estimates of gas mass using a Milky Way-like calibration factor would be underestimated by the same factor of 5 (and hence, e.g., a true gas fraction $f_{\text {gas }}=0.6$ would be misclassified as $f_{\mathrm{gas}}=0.23$ ). Submillimeter observations of thermal dust emission from our sample would further test this scenario, in combination with independent dynamical gas mass estimates (e.g., Leethochawalit et al. 2016).

\subsection{Spatial Extent of Absorbing Gas}

The spatial distribution of outflowing material is a crucial parameter for determining mass-loss rates and whether outflows exceed the local escape velocity. Although we lack direct spatial information for our sample, we can consider several independent constraints. Foremost, bulk velocities $v \simeq-150 \mathrm{~km} \mathrm{~s}^{-1}$ imply galactocentric distances

$$
d=\frac{v}{-150 \mathrm{~km} \mathrm{~s}^{-1}} \frac{t}{100 \mathrm{Myr}} \times 15 \mathrm{kpc}
$$

or $\sim 45 \mathrm{kpc}$ if the material travels at constant velocity over the characteristic star formation timescales $\mathrm{sSFR}^{-1} \simeq 300 \mathrm{Myr}$. We expect the majority of column density to be within this radius. This is supported by measurements along transverse sight lines to $z \simeq 2.2$ galaxies, in which the vast majority of $\mathrm{H} \mathrm{I}$ and low-ion absorption is concentrated within radii $<30 \mathrm{kpc}$ (Steidel et al. 2010). 
We can estimate the spatial extent of outflows in a statistical sense by comparison with quasar absorption systems. We first consider the broad population of systems identified by $\mathrm{H} \mathrm{I}$ absorption. H I column densities in the galaxy spectra correspond to DLA absorbers, which have line density $d N_{\text {DLA }} / d z \simeq 0.2$ per unit redshift at $z=2.2$, or $\sim 10^{-4}$ per Mpc (Sánchez-Ramírez et al. 2016). Defining $f_{\text {DLA }}$ as the fraction of DLAs arising in galaxy outflows similar to our sample, the cross sectional area of outflowing gas $\sigma_{\mathrm{abs}}=\pi R_{\mathrm{abs}}^{2}$ is related to the galaxy number density as

$$
n_{\mathrm{gal}}=\sigma_{\mathrm{abs}}^{-1} f_{\mathrm{DLA}} \frac{d N_{\mathrm{DLA}}}{d z} .
$$

The typical stellar mass of our galaxies suggests comoving volume densities $n_{\mathrm{gal}} \simeq 10^{-3} \mathrm{Mpc}^{-3}$ (Tomczak et al. 2014), hence

$$
R_{\mathrm{abs}} \simeq 0.2 \sqrt{f_{\mathrm{DLA}}} \mathrm{Mpc}
$$

However, we have shown that the kinematics and abundance ratios seen down the barrel in galaxy spectra are not typical of the DLA population, suggesting $f_{\text {DLA }}$ must be small. This is supported by a range of studies indicating that the majority of DLAs at $z \sim 2$ are not associated with massive galaxies (e.g., Fumagalli et al. 2010; Kulkarni et al. 2010; Pérez-Ràfols et al. 2018). Absorption kinematics are particularly constraining in this regard. All galaxies except one in our sample have characteristic $\Delta v_{90} \gtrsim 250 \mathrm{~km} \mathrm{~s}^{-1}$. Since we observe only the blueshifted and systemic gas, the value toward a background source is likely doubled: $\Delta v_{90} \gtrsim 500 \mathrm{~km} \mathrm{~s}^{-1}$. None of the 41 DLAs within the complete XQ-100 sample show such broad velocities, and only a few exhibit the distinct ion abundance patterns of our sample. If we suppose that one of the XQ-100 DLAs is associated with the absorption systems seen in the galaxy spectra, then $f_{\text {DLA }}=1 / 41$ gives a characteristic radius $R_{\text {gal }}=30 \mathrm{kpc}$. Equivalently, if the absorption we observe arises from within $R \leqslant 30 \mathrm{kpc}$ from the galaxies, then we expect $2.5 \%$ of DLAs to be associated with such outflows. The scarcity of similar systems among DLA populations is therefore explained if the bulk of absorbing material is at impact parameters within a few tens of kiloparsecs.

We next consider which subset of quasar absorption systems are most likely to be associated with galaxy outflows. Metal ion equivalent widths are a powerful discriminant, as the chemical enrichment and broad velocities of outflows give rise to large equivalent widths compared to typical DLAs. Of the various metal transitions, Mg II $\lambda \lambda 2796,2803$ has been extensively studied and provides a good basis for comparison with quasar absorbers. Moreover, a variety of studies have associated strong Mg II absorption systems at $z \sim 1$ with galactic outflows (e.g., Zibetti et al. 2005; Bouché et al. 2007; Ménard \& Chelouche 2009; Bordoloi et al. 2011; Lundgren et al. 2012). We estimate $\mathrm{Mg}$ II equivalent widths of our sample from velocity-integrated low-ion covering fraction profiles, defining

$$
W_{\mathrm{vel}}=\int f_{\mathrm{cov}} d v
$$

The values for our sample are shown in Figure 19. $W_{\text {vel }}$ is versatile in that a saturated transition at wavelength $\lambda$ has

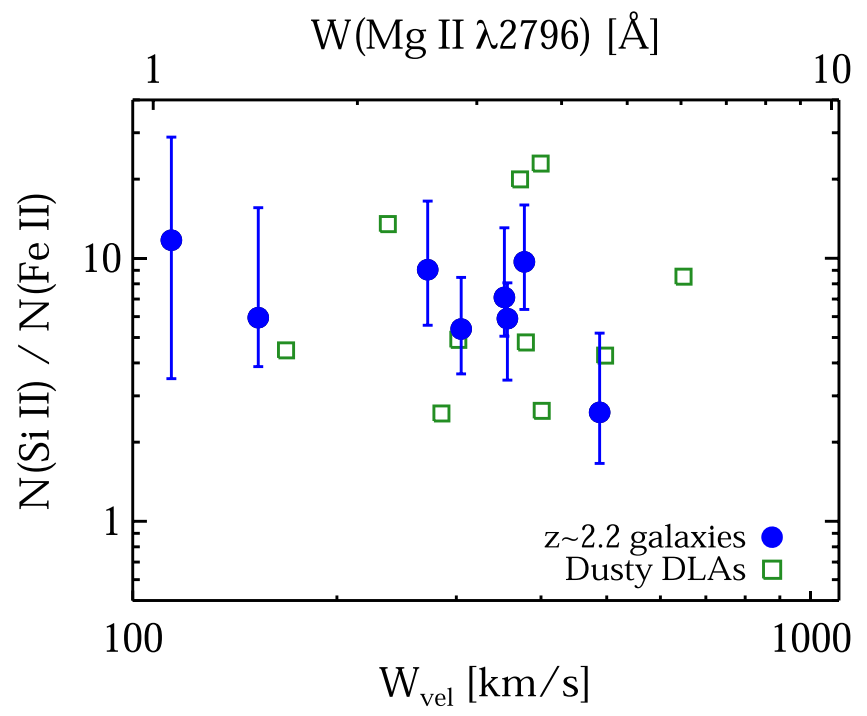

Figure 19. Equivalent width of saturated low-ion transitions in our sample. We define $W_{\text {vel }}$ from the covering fraction profile, which is in turn directly proportional to the equivalent width of saturated transitions (Equations (22) and (23)). Mg II rest-frame equivalent widths are given on the upper axis as an example, and the Ma et al. (2017) "dusty DLA" sample is shown for comparison. Absorption profiles in the galaxy sample are comparable to these dusty DLAs, with typical $W(\mathrm{Mg}$ II $\lambda 2796) \simeq 3 \AA$ A. Such strong Mg II absorbers are $\sim 10$ times less abundant than DLAs at these redshifts (Zhu \& Ménard 2013). Metal ion equivalent widths are therefore useful for identifying the rare subset of quasar absorption systems that trace galactic outflows.

equivalent width given by

$$
W=\frac{W_{\mathrm{vel}}}{c} \times \lambda
$$

Although $\mathrm{Mg}$ II is not directly measured in most cases, we expect the strong $\lambda 2796$ transition to be optically thick based on other low-ion column densities (confirmed in the case of CSWA 141). Therefore, we use Equation (23) to calculate $W$ (Mg II 12796), with results given on the upper axis in Figure 19. This demonstrates that the low-ion profiles in our sample correspond to rest-frame $W(\mathrm{Mg}$ II $\lambda 2796) \simeq 3 \AA$. As we only observe the blueshifted absorption, equivalent widths seen toward a background source would be higher $(\sim 5 \AA$; e.g., Steidel et al. 2010). Analogous to Equation (21), we can now constrain the spatial extent of outflows based on the incidence of strong $\mathrm{Mg}$ II absorbers seen in quasar spectra. Adopting a conservative threshold $W(\mathrm{Mg}$ II $\lambda 2796) \geqslant 3.5 \AA$ (with line density $d N_{\mathrm{Mg} \text { II }} / d z \simeq 0.01$ at $z=2.2$; Zhu \& Ménard 2013) gives

$$
R_{\mathrm{abs}} \simeq 50 \sqrt{f_{\mathrm{Mg} \mathrm{II}}} \mathrm{kpc}
$$

Here, $f_{\mathrm{Mg} \text { II }}$ is the fraction of such $\mathrm{Mg}$ II absorbers arising in outflows similar to our sample. Bouché et al. (2012) have carried out a search for star-forming galaxies within $\sim 100 \mathrm{kpc}$ of strong Mg II absorbers at $z \simeq 2$, with a success rate of $4 / 20$. The impact parameters of $6-26 \mathrm{kpc}$ of associated galaxies further suggest $f_{\mathrm{Mg} \text { II }} \sim 0.2$ and $R_{\mathrm{abs}} \simeq 25 \mathrm{kpc}$. Comparison with Equation (21) shows clearly that these galactic outflows comprise at most a few percent of the DLA population, considering only their low-ion equivalent widths. Furthermore, 
we reaffirm that the outflows are confined to at most $\sim 50 \mathrm{kpc}$ and quite possibly to much smaller radii.

If the reasoning of this section is correct, we expect that the subset of quasar absorption systems with properties similar to our sample (e.g., in terms of column density, abundance ratios, velocity spread, and equivalent width of low ions) are associated with star-forming galaxies at small angular separation. In Section 6.8, we discuss further evidence for small characteristic impact parameters from an independent chemical yield argument. The closest analogs in Figures 11, 13, and 19 are the $2175 \AA$ dust absorbers described by Ma et al. (2017). Recently, Ma et al. (2018) reported Hubble Space Telescope follow-up of one such system which reveals the $z=2.1$ host galaxy at an impact parameter of only $5.5 \mathrm{kpc}$, supporting this hypothesis. We propose that other similar quasar absorption systems are associated with hosts at scales $R \lesssim 30 \mathrm{kpc}$ or equivalently $\lesssim 3$." 5 , which can be further tested via targeted searches for host galaxy emission lines using integral field spectrographs (e.g., Bouché et al. 2012). We emphasize that these systems represent only a small percentage of the quasar absorber population but can be efficiently identified on the basis of metal absorption properties.

\subsection{Mass-loss and Recycling Rates}

Mass-loss rates can be estimated from the column density and velocity profiles of outflowing material. The accuracy is limited by the uncertainty in the geometry of the outflow, in particular its distribution in galactocentric radius, which we discuss above. Here, we will adopt a toy model shell geometry characterized by a shell radius $R$, width $\Delta R$, and number density $n=\rho / \mu$. Here, $\rho$ is the mass density and $\mu \simeq 1.4$ AMU is the mean ion mass assuming the outflow is dominated by atomic or ionized $\mathrm{H}$ and $\mathrm{He}$. At radius $R$, the flux of mass in a given time interval $d t$ is

$$
d M=4 \pi R^{2} n \mu \nu d t
$$

where $v$ is the outflow velocity. The mass-loss rate is

$$
\dot{M}=4 \pi R^{2} n \mu v=4 \pi R \frac{R}{\Delta R} N \mu v
$$

with column density $N=n \times \Delta R$ for the shell geometry. This is equivalent to the formalism of Pettini et al. (2000) with $R / \Delta R=3$. We note that while a spherically symmetric geometry is appropriate for an ensemble average, individual galaxy outflows are presumably not isotropic. Consequently, the outflow major axes for individual galaxies will extend beyond the radial distances in Equations (25) and (26) by an amount dependent on the degree of collimation.

The column density $N$ in Equation (26) represents the total of all ionic and molecular species. In practice, we can redefine $N$ in terms of a single species and its abundance. Hydrogen would be a natural choice as it dominates the mass. In our case, the velocity and column density profiles are more robustly constrained for metal ions. Fe II is the most uniformly welldetermined ion and the best available tracer of H I. Adopting Fe II as a reference yields

$$
\begin{aligned}
\dot{M}= & -\frac{R}{5 \mathrm{kpc}} \frac{R}{\Delta R} \frac{N_{\mathrm{Fe} \mathrm{II}}}{10^{14.5} \mathrm{~cm}^{-2}} \frac{N_{\mathrm{Fe}}}{N_{\mathrm{FeII}}} 10^{-(1+[\mathrm{Fe} / \mathrm{H}])} \\
& \times \frac{v}{-150 \mathrm{~km} \mathrm{~s}^{-1}} \times 10 M_{\odot} \mathrm{yr}^{-1}
\end{aligned}
$$

where the negative value implies mass loss. The main uncertainty is due to galactocentric distance mean $(R)$ and range $(\Delta R)$. We expect these to be $\lesssim 30 \mathrm{kpc}$ as discussed above and larger than the characteristic galaxy sizes ( $\gtrsim 3 \mathrm{kpc})$, hence we can obtain results to within an order of magnitude. The product of depletion (via $\mathrm{Fe} / \mathrm{Fe}$ II) and metallicity correction terms is actually well defined from our results in Equation (13) and represents a factor of $\sim 4$. Estimating the remaining terms to be of order unity, the mass-loss rates of low-ionization gas alone are $\dot{M} \sim 40 M_{\odot} \mathrm{yr}^{-1}$. This is $\sim 3 \times$ larger than SFRs of the sample. The implied mass-loading factors $\eta=\dot{M} /$ SFR are therefore at least of order unity and may be substantially higher when accounting for ionized gas phases or if the galactocentric distances are $>5 \mathrm{kpc}$.

We now turn briefly to the question of how much enriched material is being recycled back to the galaxy via a "galactic fountain" process (e.g., Bregman 1980). It appears that relatively little metal mass is recycling, given the low column densities at positive velocity (inflowing). From the analysis in Section 4, the typical mass flux $\dot{M} \propto N v$ associated with inflowing velocities is 0.13 that of the outflowing velocities. A systemic ISM component at $v \simeq 0$ would further reduce the ratio of inflow/outflow flux. On the other hand, relative inflow rates could be larger if the inflowing material is predominantly at larger distances. We would then have

$$
\frac{\dot{M}_{\text {in }}}{\dot{M}_{\text {out }}} \leqslant 0.13\left(\frac{R_{\text {in }}}{R_{\text {out }}}\right)^{2}
$$

with the upper bound corresponding to zero ISM component. We note that this result is derived from the low ions only. Larger velocity spreads for the higher ions (Figure 10) permit higher recycling rates if the inflowing component is more highly ionized. We conclude that the metal recycling rate is not more than $\sim 10 \%$ of the metal outflow rate, unless the inflowing gas is significantly farther from the galaxy or more highly ionized.

\subsection{Fraction of Metals Ejected}

The impact of outflows on galaxy chemical evolution depends on both the mass-loss rate and the relative fraction of metals that are ejected in outflows. The fraction of metals lost is better constrained than the total mass lost, as the metal budget can be derived from a galaxy's stellar population. In particular, Peeples et al. (2014) find that $\sim L^{*}$ galaxies at $z=0$ - encompassing the expected descendants of our sampleretain only $\sim 20 \%-25 \%$ of their total metals while the rest are ejected over the course of their formation histories. Our measurements provide an independent view at the peak epoch of metal production.

We can address the ejected metal fraction most readily with the $\alpha$-element $\mathrm{Si}$. The production of $\mathrm{Si}$ is dominated by corecollapse supernovae on approximately instantaneous timescales. For comparison purposes, we adopt the same Type II supernova yields as Peeples et al. (2014), such that an SFR of 1 $M_{\odot} \mathrm{yr}^{-1}$ corresponds to $0.0014 M_{\odot} \mathrm{yr}^{-1}$ of $\mathrm{Si}$ production. The typical production rate for galaxies in our sample is then $\dot{M}_{\mathrm{Si}, \mathrm{SFR}}=0.02 M_{\odot} \mathrm{yr}^{-1}$ (median). Rewriting Equation (26) in 
terms of the Si mass-loss rate, we have

$$
\begin{aligned}
\dot{M}_{\mathrm{Si}}= & 4 \pi R \frac{R}{\Delta R} N_{\mathrm{Si}} \mu_{\mathrm{Si}} v \\
= & -\frac{R}{5 \mathrm{kpc}} \frac{R}{\Delta R} \frac{N_{\mathrm{Si} \mathrm{II}}}{10^{16} \mathrm{~cm}^{-2}} \frac{N_{\mathrm{Si}}}{N_{\mathrm{Si} \mathrm{II}}} \frac{v}{-150 \mathrm{~km} \mathrm{~s}^{-1}} \\
& \times 0.022 M_{\odot} \mathrm{yr}^{-1} .
\end{aligned}
$$

We measure a median $N_{\mathrm{Si} \mathrm{II}}=3.4 \times 10^{15} \mathrm{~cm}^{-2}$ and infer Si depletions of $\sim 0.25$ dex from the discussion above (i.e., $\frac{N_{\mathrm{Si}}}{N_{\mathrm{Si}} \text { II }}=1.8$ accounting only for the low ions and associated dust). Adopting these values together with $v=-150 \mathrm{~km} \mathrm{~s}^{-1}$, we can express the median instantaneous fraction of Si metal loss as the ratio of ejection in outflows to production in supernovae:

$$
f_{\mathrm{ej}, \mathrm{Si}}=\frac{\dot{M}_{\mathrm{Si}, \mathrm{ej}}}{\dot{M}_{\mathrm{Si}, \mathrm{SFR}}}=0.6 \frac{R}{5 \mathrm{kpc}} \frac{R}{\Delta R} .
$$

In other words, $\sim 60 \%$ of the $\alpha$-element metal budget is ejected in the low-ion and solid phases alone! While Si I and Si IV are subdominant compared to Si II, both Si III and a more highly ionized outflow phase could further increase $f_{\mathrm{ej}, \mathrm{Si}}$. Given the limited remaining metal budget, however, Equation (30) affirms the importance of low ions and dust in tracing a large fraction of the total outflow mass.

Equation (30) shows clearly that a substantial fraction and perhaps the majority of all $\alpha$-elements generated by supernovae are being ejected in outflows, with a low-ionization phase dominating the column density. This is in good agreement with the FIRE cosmological simulations discussed by Muratov et al. (2017), who explicitly quantified the thermal state of heavy elements. At similar stellar masses and $z \simeq 2.2$, these authors find that a majority of metals are ejected from galaxies, with $\sim 70 \%$ of CGM metals in a low-ionization phase (see in particular Figure 12 of Muratov et al. 2017). These simulations have mass-loading factors of $\sim 3-5$ for stellar masses and redshifts similar to our sample (Muratov et al. 2015), similar to our results in Section 6.7. Notably, the simulations also reproduce observed mass-metallicity relations via this mass and metal loss. Although the large fraction found in Equation (30) may seem extreme, it is in fact expected both from simulations and from the $z=0$ metal budget accounting by Peeples et al. (2014).

Radial distribution factors are left as variables in Equation (30) since they are not directly measured. However, we can derive interesting limits on the spatial extent and other outflow properties from the simple metal budget argument used here. While not strictly necessary at all times, the total metal content of galaxies generally increases over time, which requires $f_{\text {ej }}<1$. Applying this condition gives a limit to the characteristic radius

$$
R \times \frac{R}{\Delta R}<8 \mathrm{kpc} .
$$

Such small radial scales support our conclusions from Section 6.6 and explain why the outflow properties in our sample are so rarely seen in quasar absorber surveys. This result motivates a need for sight lines at small impact parameters $(\lesssim 10 \mathrm{kpc})$ in order to probe the majority of outflowing mass. By the same argument, depletion factors must be $\delta_{\mathrm{Si}} \lesssim 0.5$ (and correspondingly $\delta_{\mathrm{Fe}} \lesssim 1.1$ ) to keep $f_{\mathrm{ej}}<1$, with the precise constraint limited by the unknown radial extent. The presence of metals in phases other than the low ions and solids is likewise constrained to be small (i.e., column density similar to or less than the low ions). These constraints further justify our focus on the low-ion phase as likely representative of the total outflow composition.

In summary, accounting of the metal budget-comparing observed column densities with expected stellar yieldsprovides strong constraints on the radial extent, dust depletion, and any unseen ionization states of outflowing material. Our measurements indicate a theoretically supported picture in which the majority of heavy elements are ejected in a predominantly low-ionization outflow, which regulates galactic chemical evolution.

\subsection{Diversity within the Galaxy Sample}

We have focused the discussion largely on the mean characteristics of the sample, and we now consider the extent to which individual galaxies deviate from the average in various properties. Low-ion column density ratios are remarkably consistent within measurement uncertainties across the sample (Figure 12). Mean low-ion velocities vary but are uniformly within 0 to $-200 \mathrm{~km} \mathrm{~s}^{-1}$ relative to systemic, with covering fraction profile widths spanning a narrow range $\sigma_{v} \simeq$ 150-200 $\mathrm{km} \mathrm{s}^{-1}$ (Figure 9). In contrast to the uniform kinematics and abundance ratios, the total column densities vary by a factor of $\sim 10$ within the sample. The covering fraction profiles also vary by a factor of $\sim 2$ (Figure 6).

Homogeneous low-ion abundance ratios suggest that intrinsic abundance patterns, depletion factors, and ionization factors are similar throughout the sample. This extends to previous studies, which we have shown to be remarkably consistent with our sample properties (i.e., the galaxies studied by Pettini et al. 2002; Quider et al. 2009; Dessauges-Zavadsky et al. 2010). The most significant variations are in the total inferred interstellar mass and its covering fraction. These are correlated in the sense that galaxies with higher covering fractions have higher column densities. The correlation is partially but not entirely due to our definition of $N_{\text {tot }} \propto f_{c}$. We might expect the low-ion column density to depend on ionization state, but this does not appear to be significant: the ionization indicator [Ni II/Fe II] shows no strong correlation with total column densities (Figure 12).

While we find that our sample is relatively homogeneous in many properties of the interstellar and outflowing medium, we can also give insight into the systematic trends of strong absorption properties found in stacked spectra. Perhaps most notable is the variation in the equivalent width of saturated lowion transitions, which is most strongly correlated with the Ly $\alpha$ equivalent width (Shapley et al. 2003; Jones et al. 2012; Du et al. 2018). This is clearly driven by variations in the gas covering fraction: we see a wide range in the low-ion covering fraction within the sample (sufficient to explain the variation in stacked spectra; Figure 6) yet very little range in the velocity width of strong low-ion transitions (Table 5). This supports the conclusions of our earlier work (Jones et al. 2013; Leethochawalit et al. 2016) with a larger sample. Variations in covering fraction also contribute partially to the range of column densities $N_{\text {tot }}$ as noted above. Additional trends with demographic properties such as galaxy stellar mass, SFR, 
optical extinction, and surface density are undoubtedly present, but our sample is not yet large enough to characterize these dependencies. Nonetheless, these data provide clear guidance for the interpretation of statistically significant trends from samples of hundreds to thousands of galaxies at these redshifts.

\section{Summary}

We have examined the kinematics, geometric covering fraction, and chemical composition of the interstellar medium in a sample of nine galaxies at redshifts $z=1.4-2.9$. Galaxies in our sample are gravitationally lensed but otherwise representative of the star-forming population at these redshifts. Our analysis takes advantage of optically thin absorption lines probed by high-quality spectra, necessary to study the mass distribution and relative abundances of different elements in both the gaseous and solid ISM phases, as well as to guide the interpretation of results from lower-resolution composite or individual galaxy spectra. Our main results are as follows.

1. Covering fraction: The low-ionization gas exhibits a wide range of geometric covering fractions. $f_{c}$ varies as a function of velocity, with maximum covering fractions ranging from $f_{c} \simeq 0.4$ to 1 for individual galaxies in our sample. Most galaxies have non-uniform covering at all velocities $\left(f_{c}<1\right)$. Variations in the covering fraction are able to explain the demographic trends measured from stacked spectra of galaxies at similar redshifts, such that a lower covering fraction is correlated with a higher Ly $\alpha$ equivalent width and lower reddening (i.e., dust column density). Correlations with $\mathrm{Ly} \alpha$ and reddening arise naturally from the presence of $\mathrm{H}$ I and dust, respectively, in the low-ionization phase.

2. Kinematics: We use optically thin transitions to measure gas column densities as a function of velocity. In contrast to the range of covering fractions, low-ionization gas kinematics show only a modest variation within the sample. Approximately $80 \%$ of the low-ion phase is associated with a net outflow, with absorption detected at speeds of at least $400 \mathrm{~km} \mathrm{~s}^{-1}$ in every galaxy. Mean velocities along the line of sight are $\simeq-150 \mathrm{~km} \mathrm{~s}^{-1}$. We find little or no significant metal recycling $(\lesssim 10 \%$, if inflows and outflows are similar in distance and ionization). Strong absorption transitions are systematically $\sim 2 \times$ broader in velocity width than the column density distribution but have consistent centroids, such that saturated transitions can be used to accurately measure mean bulk velocities.

3. Composition: Low-ion column density ratios provide measurements of the metallicity, dust depletion, and nucleosynthetic abundance patterns. Column density ratios in the outflowing gas show remarkably little dispersion within the sample, yet they are distinct from other well-characterized astrophysical sources (e.g., DLAs, nearby galaxies' ISM, and stellar abundances). We infer that the outflowing medium is characterized by subsolar metallicity, $\alpha$-enhancement, and moderate dust depletion $\left([\mathrm{Fe} / \mathrm{H}] \simeq-0.9,[\mathrm{Si} / \mathrm{Fe}] \simeq 0.2\right.$, and $\delta_{\mathrm{Fe}} \simeq-0.6$ dex). Depletion is highly important in determining abundance patterns from gas-phase transitions: we find that the majority of $\mathrm{Fe}$ and $\mathrm{Ni}$ atoms are in the solid state, along with approximately half of the Si. Dust-to-gas ratios are lower than in the Milky Way ISM, suggesting caution in calibrating thermal dust emission to estimate ISM gas masses. While these results have some degeneracy, the abundance patterns are in good agreement with metal-poor and $\alpha$-enhanced stars in the Milky Way thick disk and bulge. We consider this evidence for in situ thick disk and/or bulge formation at these redshifts (corroborated by kinematics of the star-forming gas available for a subset of the CSWA sample). Our interstellar data are in good agreement with recent measurements of nebular and stellar abundances from composite spectra by Steidel et al. (2016), provided that $[\mathrm{O} / \mathrm{Si}]$ is highly supersolar.

4. Mass and metal outflow rates: We determine mass-loss rates and mass-loading factors to within an order of magnitude accuracy for the low-ionization phase, limited by uncertainty in the radial distribution of the outflowing gas. Typical mass-loss rates are $\sim 40 M_{\odot} \mathrm{yr}^{-1}$ with corresponding mass-loading factors $\dot{M}_{\text {out }} / \mathrm{SFR} \sim 3$. Total mass-loss rates may be somewhat larger when accounting for highly ionized gas. Comparing the instantaneous rates of metal production by Type II supernovae versus metal loss in outflows, we find that of order half of all metals produced are being ejected in the low-ionization and solid phases alone, consistent with censuses of the metal budget for descendant $z=0$ galaxies. Requiring the metal production rate to be greater than the ejection rate, we find that the characteristic outflow radii must be $\lesssim 10 \mathrm{kpc}$. Such small impact parameters are rarely sampled with background sight lines, explaining the scarcity of quasar absorption systems with similar physical properties.

This work represents the first characterization of the ISM and outflow properties based on column densities measured for a sample of galaxies at $z \simeq 2-3$, when star formation and associated feedback are most active. Such information is essential for understanding the role of feedback and large-scale outflows in regulating gas content, star formation, and metallicity of galaxies and their surrounding medium. We envision a number of feasible avenues to further extend and improve upon our results in the near future. First, knowledge of the chemical compositions will benefit from column density measurements of additional ions especially at blue wavelengths $\left(\lambda_{\text {rest }}<1260 \AA\right.$ ), most notably $\mathrm{H}$ I which is available only for four of the nine galaxies in our sample. Metal ions with a range of ionization and depletion factors will help to more accurately correct for these effects and to better probe abundance patterns of multiple nucleosynthetic groups (e.g., the iron peak, $\alpha$ capture, and secondary elements). N I, S II, P II, Cr II, Mn II, and $\mathrm{Zn}$ II are all achievable with moderately deep spectroscopy. Volatile elements such as $\mathrm{S}$ and $\mathrm{Zn}$ are of particular interest (and we find that even low $\mathrm{S} / \mathrm{N}$ measurements of $\mathrm{Zn}$ II are valuable).

Mass-loss rates and metal-loss fractions can be improved substantially with better information on the spatial distribution of outflows (i.e., their radial extent). We find that the majority of the column density is likely within galactocentric distances of order $10 \mathrm{kpc}$. One possible way forward is to specifically target these small impact parameters with background sight lines (e.g., QSOs); such alignments are rare, and current surveys typically do not probe this regime. In our view, a more promising approach is to spatially map fluorescent emission from fine-structure transitions of $\mathrm{Si} \mathrm{II}^{*}$ and $\mathrm{Fe} \mathrm{II}^{*}$, which 
originate in the outflowing gas (e.g., Jones et al. 2012). Recently, the first such measurement at $z>1$ shows a Fe II half-light radius of $\simeq 4 \mathrm{kpc}(\sim 0 \prime \prime 5$; Finley et al. 2017), supporting our conclusions. Areal magnification from gravitational lensing is needed to adequately sample these scales with seeing-limited instruments. Our lensed sample is ideal for mapping fine-structure emission with the newly commissioned Keck Cosmic Web Imager (KCWI), which we are now pursuing.

Finally, while this work quadruples the previously available sample, we ultimately seek equivalent data for larger samples of galaxies spanning a range of redshift and physical properties. Enlarged samples are needed to firmly establish demographic trends such as the mass-loading factor as a function of stellar mass. Characterizing these scaling relations will allow us to better understand origins of the stellar-to-halo mass relation, mass-metallicity relation, and other fundamental properties of the galaxy population. In the short term, gravitational lensing continues to be a productive approach. Bright lensed galaxy samples are growing thanks to both wide-area sky surveys (e.g., the Dark Energy Survey and Pan-STARRS; Chambers et al. 2016; Nord et al. 2016) and targeted lensing surveys (e.g., eMACS and RELICS; Ebeling et al. 2013; Salmon et al. 2017). Nonlensed galaxies may become accessible with $\sim 100 \mathrm{hr}$ integration times using multiobject spectrographs, and $30 \mathrm{~m}$ class optical telescopes will more easily reach the bright field galaxy population. However, we note that current ultradeep surveys such as VANDELS (Pentericci et al. 2018) and the MUSE UDF (Bacon et al. 2017) lack the spectral resolution and blue wavelength coverage needed for this work. We emphasize that upcoming $30 \mathrm{~m}$ class telescopes will require instrumentation with moderate to high spectral resolving power and good throughput in the near-UV $(R \gtrsim 5000$ and $\lambda<4000 \AA$, ideally reaching the atmospheric cutoff) to optimally address chemical abundance patterns at $z \simeq 2$.

We thank Ramesh Mainali for providing measurements of the stellar masses; George Becker, Sarah Loebman, and Crystal Martin for several enlightening conversations; and the referee for several suggestions which considerably improved the discussion and clarity of the manuscript. R.S.E. acknowledges financial support from the European Research Council from an Advanced Grant FP7/669253. D.P.S. acknowledges support from the National Science Foundation through the grant AST1410155. T.J. acknowledges support from NASA through Hubble Fellowship grant HST-HF2-51359.001-A awarded by the Space Telescope Science Institute, which is operated by the Association of Universities for Research in Astronomy, Inc., for NASA, under contract NAS 5-26555. This work is based on data obtained at the W. M. Keck Observatory, which is operated as a scientific partnership among the California Institute of Technology, the University of California, and the National Aeronautics and Space Administration. The Observatory was made possible by the generous financial support of the W. M. Keck Foundation. We wish to acknowledge the very significant cultural role and reverence that the summit of Maunakea has within the indigenous Hawaiian community. We are most fortunate to have the opportunity to conduct observations from this sacred mountain, and we respectfully say mahalo.

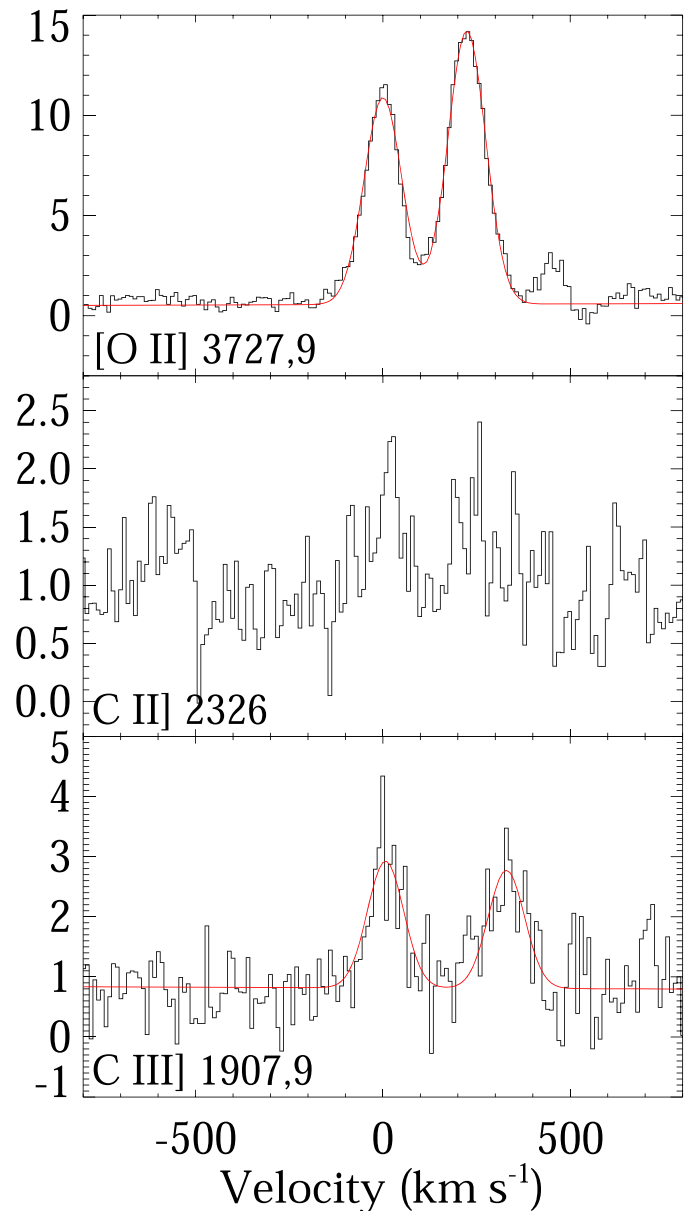

Figure 20. Features used to derive the systemic redshift of CSWA 141. Red lines show the best-fit double Gaussian functions to [O II] and C III].

\section{Appendix Systemic Redshifts}

In this section, we briefly describe the spectral features used to measure systemic redshifts for individual sources. Redshifts were measured from line centroids determined from Gaussian fits, compared to reference vacuum transition wavelengths from the NIST Atomic Spectra Database ${ }^{6}$ (Kramida et al. 2016). In cases of emission line doublets, we simultaneously fit both lines assuming a common redshift and velocity dispersion to maximize precision. This applies to $\mathrm{O}$ III] $\lambda \lambda 1661,6, \mathrm{C}$ III] $\lambda \lambda 1907,9$, and [O II] $\lambda \lambda 3727,9$. Similarly, in several cases we fit stellar photospheric absorption lines simultaneously with a common redshift and velocity dispersion in order to improve the significance and fidelity of redshift measurements. For this analysis, we use the relatively unblended $\mathrm{Si}$ III $\lambda 1294, \mathrm{Si}$ III $\lambda 1417, \mathrm{~S}$ V $\lambda 1501, \mathrm{~N}$ IV $\lambda 1718$, and C III $\lambda 2297$ features.

In several cases, we also measure redshifts of fine-structure emission lines arising from $\mathrm{Si}$ II $^{*}$ and Fe II* (where “*” denotes fluorescent emission to excited fine-structure ground states). These lines are thought to arise from de-excitation into the excited ground state following absorption by interstellar gas (e.g., Prochaska et al. 2011; Jones et al. 2012). Since resonant absorption transitions of Si II and Fe II typically show a much larger velocity extent than stars and $\mathrm{H}$ II regions, the corresponding fine-structure transitions do not necessarily trace

\footnotetext{
6 http://physics.nist.gov/asd
} 


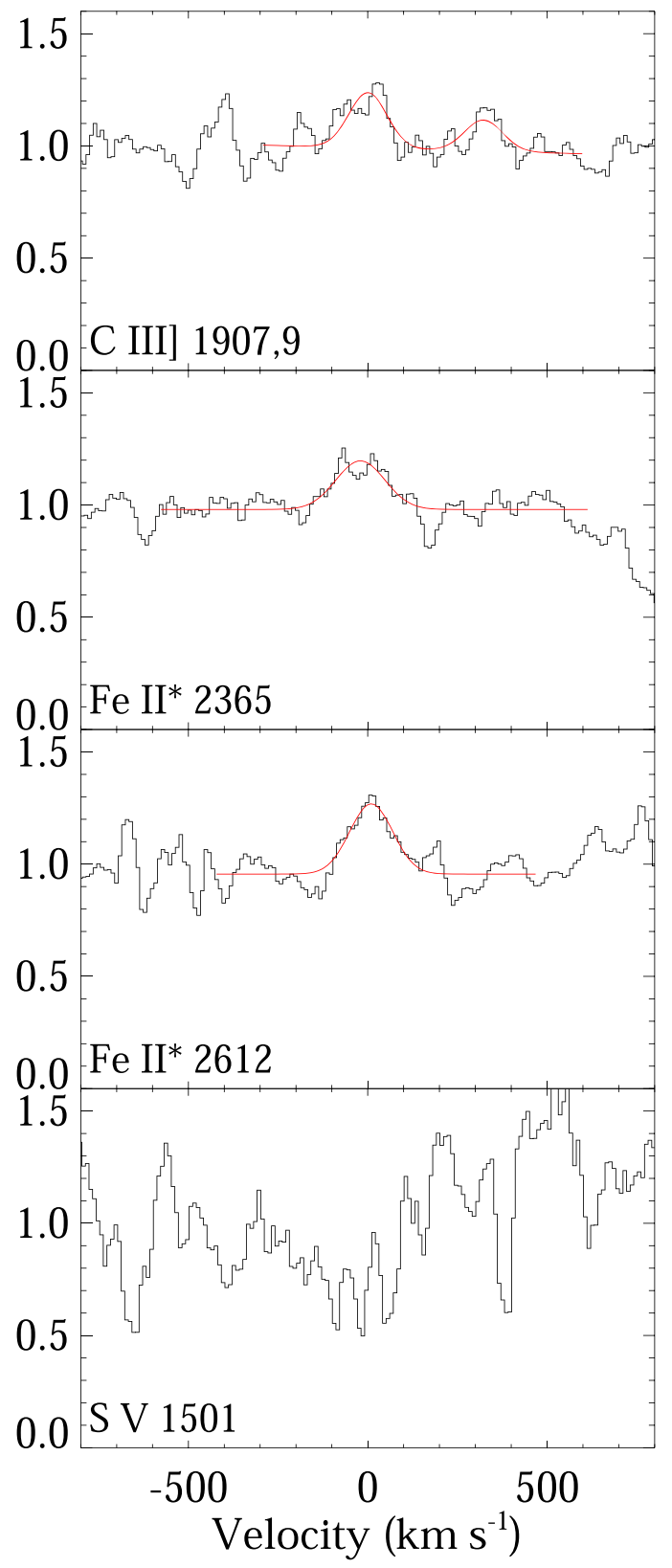

Figure 21. Features used to determine the systemic redshift of CSWA 103.

the systemic velocity. However, their centroids are typically consistent with the systemic redshift (within $\sim 100 \mathrm{~km} \mathrm{~s}^{-1}$; e.g., Shapley et al. 2003; Jones et al. 2012; Kornei et al. 2013) as expected from outflow models (Prochaska et al. 2011). In this sample, we find fine-structure line centroids within $50 \mathrm{~km} \mathrm{~s}^{-1}$ of the adopted systemic redshift in all cases with good $\mathrm{S} / \mathrm{N}$, with mean and median offsets of $<10 \mathrm{~km} \mathrm{~s}^{-1}$. These lines therefore provide a consistency check on the accuracy of redshifts derived from noisier features.

In Figures 20 through 28, we show a subset of the features used to derive systemic redshifts for each source. Line profiles are plotted as a function of velocity for the sake of comparison with interstellar absorption (e.g., Figure 6). For a subset of the features, we also show best-fit Gaussian profiles as red lines.

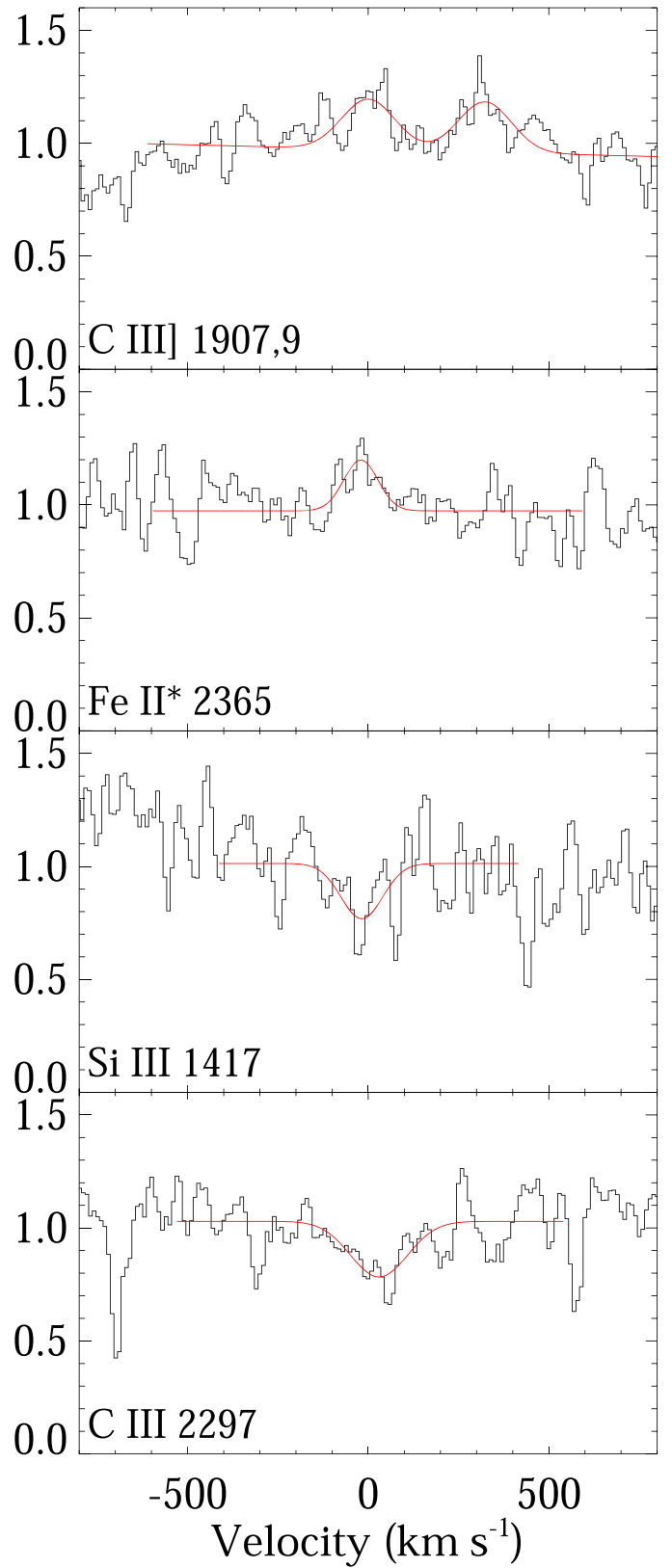

Figure 22. Features used to determine the systemic redshift of CSWA 19.

\section{A.1. CSWA 141}

The redshift of CSWA 141 is well determined from several strong nebular emission lines. The most prominent of these is [O II] $\lambda \lambda 3727,9$, which we fit with a double Gaussian function as described above. This yields the most precise redshift measurement in the sample due to the high $\mathrm{S} / \mathrm{N}$. The systemic feature with the second-highest $\mathrm{S} / \mathrm{N}$ is the $\mathrm{C}$ III] $\lambda \lambda 1907,9$ doublet. The redshift and velocity dispersion measured from $\mathrm{C}$ III] are in good agreement with [O II], with differences of $\Delta v_{\text {sys }}=7 \pm 4 \mathrm{~km} \mathrm{~s}^{-1}$ and $\Delta \sigma=-2 \pm 2 \mathrm{~km} \mathrm{~s}^{-1}$.

\section{A.2. CSWA 103}

The most significant systemic feature in the spectrum of CSWA 103 is the C III] $\lambda \lambda 1907,9$ doublet. The redshift is 


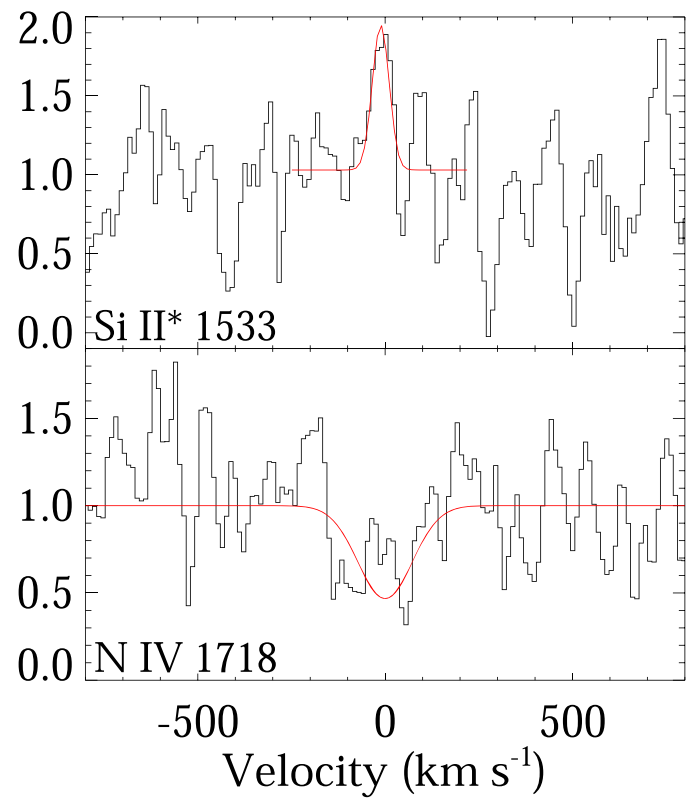

Figure 23. Features used to determine the systemic redshift of CSWA 40.

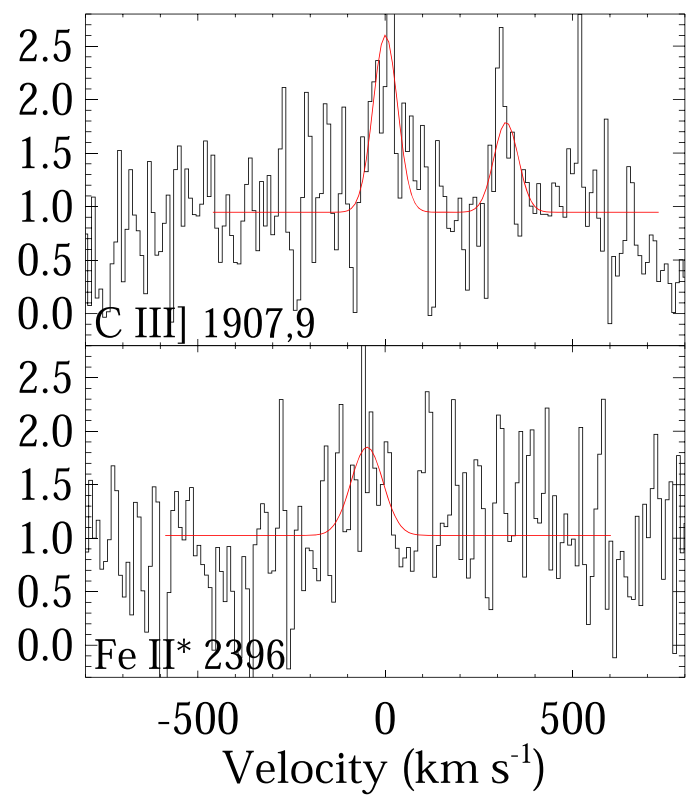

Figure 24. Features used to determine the systemic redshift of CSWA 2.

corroborated by the detection of fine-structure Fe $\mathrm{II}^{*}$ emission lines with consistent line centroids $\left(<20 \mathrm{~km} \mathrm{~s}^{-1}\right.$ offset in velocity). There is possible weak photospheric absorption from $\mathrm{S} \mathrm{V}$ although we are unable to measure a centroid for this feature.

\section{A.3. CSWA 19}

We adopt the redshift of CSWA 19 measured from C III] $\lambda \lambda 1907,9$, which is consistent with the Fe II* $\lambda 2365$ finestructure emission line as well as the photospheric absorption. Fits to the individual photospheric lines Si III 1417 and C III 2297 give centroids that are offset by -18 and $+30 /$ respectively.

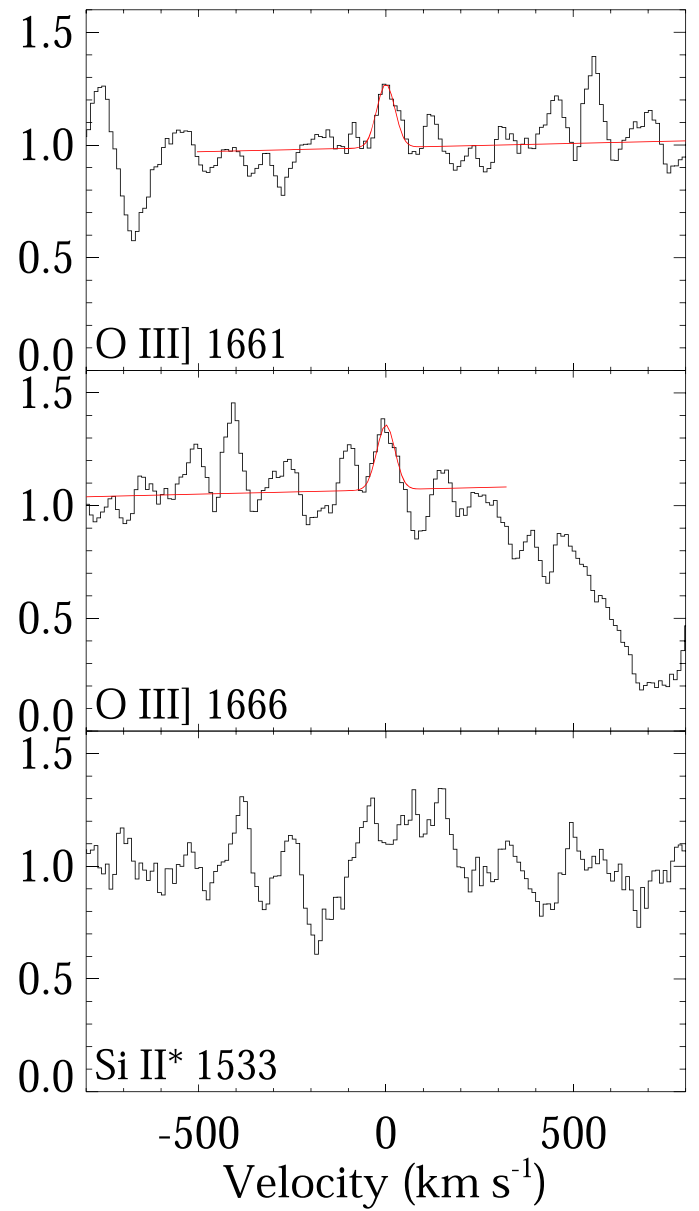

Figure 25. Features used to determine the systemic redshift of CSWA 128 Absorption at $>500 \mathrm{~km} \mathrm{~s}^{-1}$ relative to $\mathrm{O}$ III] $\lambda 1666$ is due to Al II $\lambda 1670$.

\section{A.4. CSWA 40}

We measure the redshift of CSWA 40 from a combined fit to the photospheric absorption lines Si III $\lambda 1294$, Si III $\lambda 1417$, S V $\lambda 1501, \mathrm{~N}$ IV $\lambda 1718$, and C III $\lambda 2297$. We also identify possible Si II* emission at the same redshift but with a narrower line profile.

\section{A.5. CSWA 2}

We adopt the redshift of CSWA 2 measured from C III] $\lambda \lambda 1907,9$, which is the only feature for which we are able to measure a reliable systemic redshift. We identify possible Fe II $\lambda 2396$ emission with a velocity offset of $-48 \mathrm{~km} \mathrm{~s}^{-1}$.

\section{A.6. CSWA 128}

We adopt the redshift of CSWA 128 measured from O III] $\lambda \lambda 1661,6$, which is the only feature for which we are able to measure a reliable systemic redshift although the significance of the fit is modest $(7 \sigma)$. We identify $\mathrm{Si} \mathrm{II}^{*} \lambda 1533$ emission at similar velocities. Photospheric absorption features are not significantly detected despite the $\operatorname{good} \mathrm{S} / \mathrm{N}$ of this spectrum.

\section{A.7. CSWA 164}

We measure the redshift of CSWA 164 from a combined fit to the photospheric absorption lines Si III $\lambda 1294$, Si III $\lambda 1417$, 


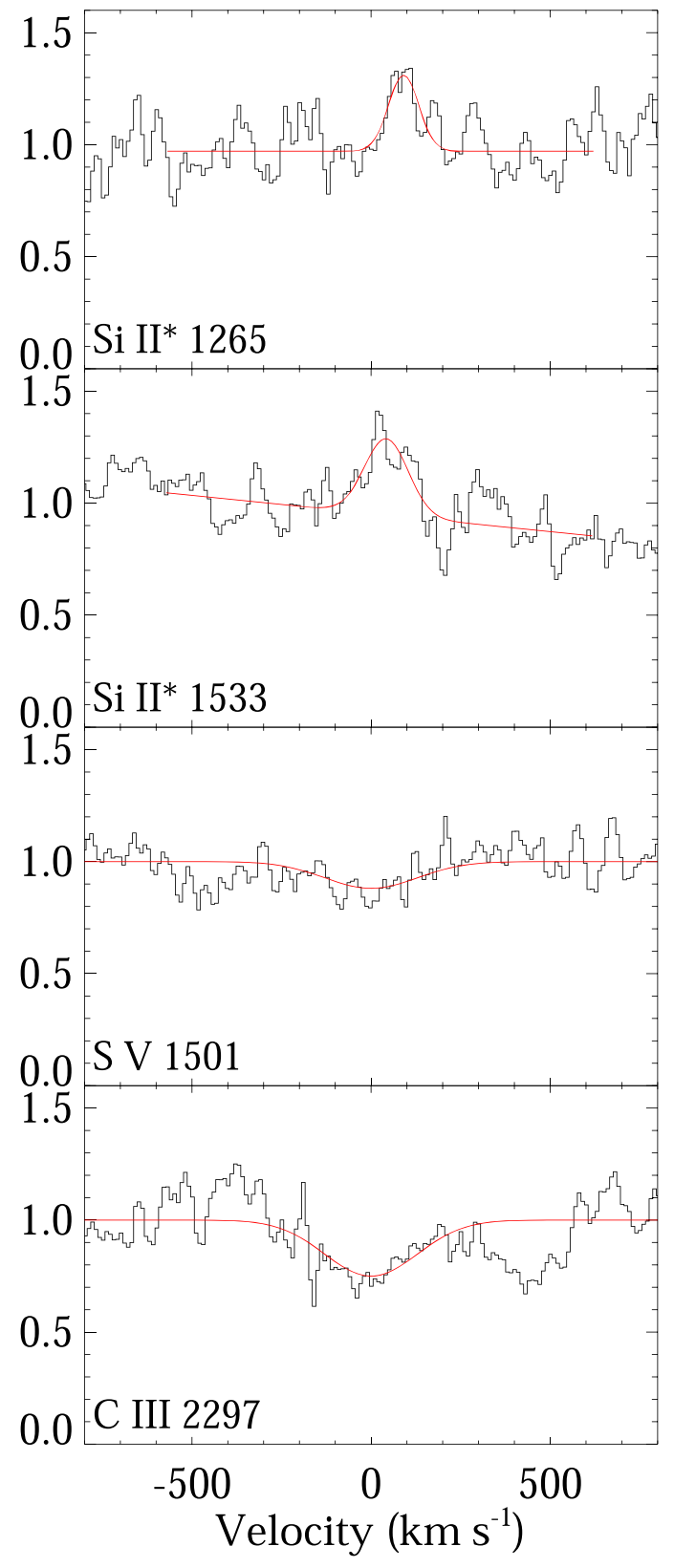

Figure 26. Features used to determine the systemic redshift of CSWA 164. The continuum slope near $\mathrm{Si} \mathrm{II}^{*} \lambda 1533$ is due to broad stellar P-Cygni C IV $\lambda \lambda 1548,51$ absorption.

S V $\lambda 1501, \mathrm{~N}$ IV $\lambda 1718$, and C III $\lambda 2297 . \mathrm{C} \mathrm{III]} \lambda \lambda 1907,9$ is also seen at low $\mathrm{S} / \mathrm{N}$ with a best-fit centroid offset by $30 \pm 15 \mathrm{~km} \mathrm{~s}^{-1}$.

\section{A.8. CSWA 39}

We adopt the redshift of CSWA 39 measured from C III] $\lambda \lambda 1907,9$ because of its relatively high $\mathrm{S} / \mathrm{N}$. The photospheric absorption lines are in good agreement, and we also weakly

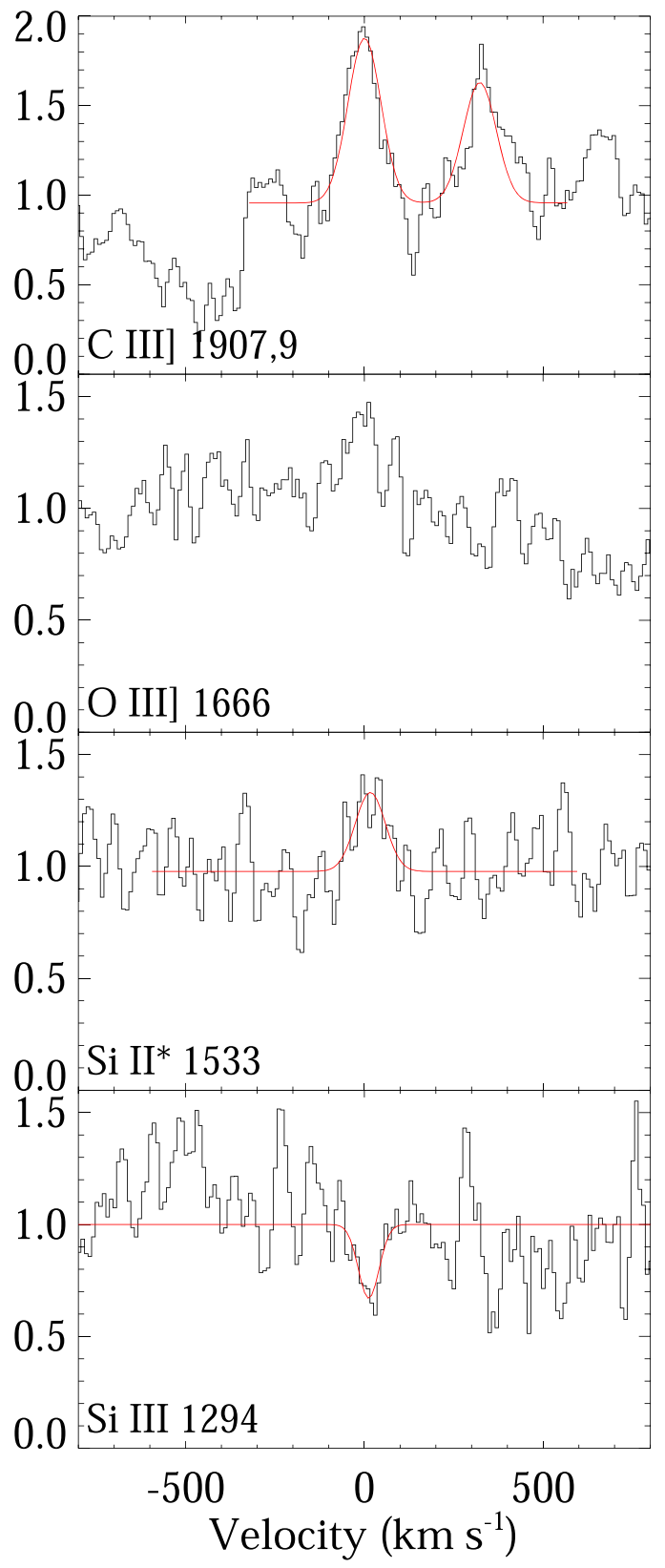

Figure 27. Features used to determine the systemic redshift of CSWA 39.

detect $\mathrm{O}$ III] $\lambda \lambda 1661,6$ and $\mathrm{Si}$ II $^{*}$ emission with velocity offsets of $\sim 15 \mathrm{~km} \mathrm{~s}^{-1}$.

\section{A.9. CSWA 38}

We measure the redshift of CSWA 38 from a combined fit to the photospheric absorption lines Si III $\lambda 1294$, Si III $\lambda 1417$, S V $\lambda 1501$, and N IV $\lambda 1718$. C III $\lambda 2297$ is excluded from the fit due to the low $\mathrm{S} / \mathrm{N}$ of the spectrum at that wavelength. We also identify Si II ${ }^{*} \lambda 1533$ and possible C III] $\lambda \lambda 1907,9$ emission at similar redshifts. 


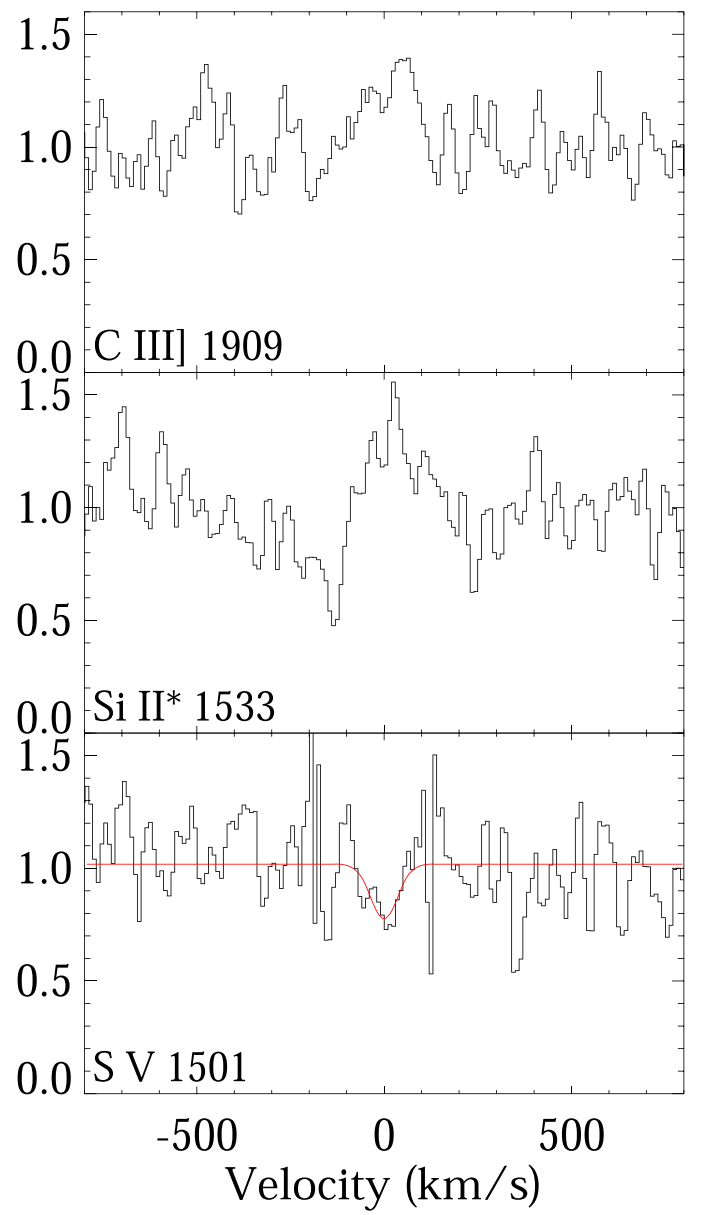

Figure 28. Features used to determine the systemic redshift of CSWA 38.

\section{ORCID iDs}

Tucker Jones (ㄴ) https://orcid.org/0000-0001-5860-3419

\section{References}

Adibekyan, V. Z., Sousa, S. G., Santos, N. C., et al. 2012, A\&A, 545, A32 Asplund, M., Grevesse, N., Sauval, A. J., \& Scott, P. 2009, ARA\&A, 47, 481 Bacon, R., Conseil, S., Mary, D., et al. 2017, A\&A, 608, A1 Becker, G. D., Sargent, W. L. W., Rauch, M., \& Carswell, R. F. 2012, ApJ, 744, 91

Belokurov, V., Evans, N. W., Moiseev, A., et al. 2007, ApJL, 671, L9 Belokurov, V., Evans, N. W., Hewett, P. C., et al. 2009, MNRAS, 392, 104 Bensby, T., Yee, J. C., Feltzing, S., et al. 2013, A\&A, 549, A147 Bensby, T., Feltzing, S., \& Oey, M. S. 2014, A\&A, 562, A71

Berg, T. A. M., Ellison, S. L., Prochaska, J. X., Venn, K. A., \& Dessauges-Zavadsky, M. 2015, MNRAS, 452, 4326

Berg, T. A. M., Ellison, S. L., Sánchez-Ramírez, R., et al. 2016, MNRAS, 463, 3021

Blanton, M. R., Bershady, M. A., Abolfathi, B., et al. 2017, AJ, 154, 28

Bordoloi, R., Lilly, S. J., Knobel, C., et al. 2011, ApJ, 743, 10

Bouché, N., Murphy, M. T., Péroux, C., et al. 2007, ApJL, 669, L5

Bouché, N., Murphy, M. T., Péroux, C., et al. 2012, MNRAS, 419, 2

Bower, R. G., Benson, A. J., \& Crain, R. A. 2012, MNRAS, 422, 2816

Bregman, J. N. 1980, ApJ, 236, 577

Buder, S., Asplund, M., Duong, L., et al. 2018, MNRAS, 478, 4513

Chambers, K. C., Magnier, E. A., Metcalfe, N., et al. 2016, arXiv:1612.05560

Choi, J., Conroy, C., Moustakas, J., et al. 2014, ApJ, 792, 95

Cowie, L. L., Songaila, A., Kim, T.-S., \& Hu, E. M. 1995, AJ, 109, 1522

Cucchiara, A., Fumagalli, M., Rafelski, M., et al. 2015, ApJ, 804, 51

Davé, R., Oppenheimer, B. D., \& Finlator, K. 2011, MNRAS, 415, 11

De Cia, A., Ledoux, C., Mattsson, L., et al. 2016, A\&A, 596, A97
Dekel, A., \& Silk, J. 1986, ApJ, 303, 39

Dessauges-Zavadsky, M., Péroux, C., Kim, T.-S., D'Odorico, S., \& McMahon, R. G. 2003, MNRAS, 345, 447

Dessauges-Zavadsky, M., D’Odorico, S., Schaerer, D., et al. 2010, A\&A, 510, A26

Draine, B. T., Dale, D. A., Bendo, G., et al. 2007, ApJ, 663, 866

Du, X., Shapley, A. E., Reddy, N. A., et al. 2018, ApJ, 860, 75

Ebeling, H., Edge, A. C., Burgett, W. S., et al. 2013, MNRAS, 432, 62

Engelbracht, C. W., Rieke, G. H., Gordon, K. D., et al. 2008, ApJ, 678, 804

Erb, D. K., Shapley, A. E., Pettini, M., et al. 2006, ApJ, 644, 813

Ferland, G. J., Chatzikos, M., Guzmán, F., et al. 2017, RMxAA, 53, 385

Finley, H., Bouché, N., Contini, T., et al. 2017, A\&A, 605, A118

Fumagalli, M., O'Meara, J. M., Prochaska, J. X., \& Kanekar, N. 2010, MNRAS, 408, 362

Gallazzi, A., Charlot, S., Brinchmann, J., White, S. D. M., \& Tremonti, C. A. 2005, MNRAS, 362, 41

Gibson, B. K., Pilkington, K., Brook, C. B., Stinson, G. S., \& Bailin, J. 2013, A\&A, 554, A47

Haardt, F., \& Madau, P. 1996, ApJ, 461, 20

Hayes, C. R., Majewski, S. R., Shetrone, M., et al. 2018, ApJ, 852, 49

Howk, J. C., \& Savage, B. D. 1999, ApJ, 517, 746

Howk, J. C., \& Sembach, K. R. 1999, ApJL, 523, L141

Jenkins, E. B., Savage, B. D., \& Spitzer, L., Jr. 1986, ApJ, 301, 355

Jenkins, E. B. 2009, ApJ, 700, 1299

Jenkins, E. B., \& Wallerstein, G. 2017, ApJ, 838, 85

Jones, T. A., Stark, D. P., \& Ellis, R. S. 2012, ApJ, 751, 51

Jones, T., Ellis, R. S., Richard, J., \& Jullo, E. 2013, ApJ, 765, 48

Jones, T. A., Ellis, R. S., Schenker, M. A., \& Stark, D. P. 2013, ApJ, 779, 52

Jones, T., Martin, C., \& Cooper, M. C. 2015, ApJ, 813, 126

Jorgenson, R. A., Murphy, M. T., \& Thompson, R. 2013, MNRAS, 435, 482

Kewley, L. J., \& Ellison, S. L. 2008, ApJ, 681, 1183

Kirby, E. N., Martin, C. L., \& Finlator, K. 2011, ApJL, 742, L25

Kornei, K. A., Shapley, A. E., Martin, C. L., et al. 2013, ApJ, 774, 50

Kramida, A., Ralchenko, Yu., Reader, J., \& ASD Team, N. I. S. T. 2016, NIST Atomic Spectra Database (version 5.4), http://physics.nist.gov/asd

Kulkarni, V. P., Khare, P., Som, D., et al. 2010, NewA, 15, 735

Law, D. R., Steidel, C. C., Shapley, A. E., et al. 2012, ApJ, 759, 29

Ledoux, C., Petitjean, P., Fynbo, J. P. U., Møller, P., \& Srianand, R. 2006, A\&A, 457, 71

Leethochawalit, N., Jones, T. A., Ellis, R. S., et al. 2016, ApJ, 820, 84

Leethochawalit, N., Jones, T. A., Ellis, R. S., Stark, D. P., \& Zitrin, A. 2016, ApJ, 831, 152

Lehner, N., Howk, J. C., Prochaska, J. X., \& Wolfe, A. M. 2008, MNRAS, 390,2

Lundgren, B. F., Brammer, G., van Dokkum, P., et al. 2012, ApJ, 760, 49

Ma, J., Ge, J., Zhao, Y., et al. 2017, MNRAS, 472, 2196

Ma, J., Brammer, G., Ge, J., Prochaska, J. X., \& Lundgren, B. 2018, ApJL, 857, L12

Madau, P., \& Dickinson, M. 2014, ARA\&A, 52, 415

Martell, S. L., Sharma, S., Buder, S., et al. 2017, MNRAS, 465, 3203

Martin, C. L., Shapley, A. E., Coil, A. L., et al. 2013, ApJ, 770, 41

Ménard, B., \& Chelouche, D. 2009, MNRAS, 393, 808

Ménard, B., Scranton, R., Fukugita, M., \& Richards, G. 2010, MNRAS, 405,1025

Milutinovic, N., Ellison, S. L., Prochaska, J. X., \& Tumlinson, J. 2010, MNRAS, 408, 2071

Morton, D. C. 1991, ApJS, 77, 119

Morton, D. C. 2000 , ApJS, 130, 403

Morton, D. C. 2003, ApJS, 149, 205

Moster, B. P., Naab, T., \& White, S. D. M. 2013, MNRAS, 428, 3121

Muratov, A. L., Kereš, D., Faucher-Giguère, C.-A., et al. 2015, MNRAS, 454, 2691

Muratov, A. L., Kereš, D., Faucher-Giguère, C.-A., et al. 2017, MNRAS, 468,4170

Nomoto, K., Kobayashi, C., \& Tominaga, N. 2013, ARA\&A, 51, 457

Nord, B., Buckley-Geer, E., Lin, H., et al. 2016, ApJ, 827, 51

Peeples, M. S., Werk, J. K., Tumlinson, J., et al. 2014, ApJ, 786, 54

Pentericci, L., Garilli, R. J. M. B., Cucciati, O., et al. 2018, arXiv:1803.07373

Pérez-Ràfols, I., Font-Ribera, A., Miralda-Escudé, J., et al. 2018, MNRAS, 473, 3019

Pettini, M., Kellogg, M., Steidel, C. C., et al. 1998, ApJ, 508, 539

Pettini, M., Steidel, C. C., Adelberger, K. L., Dickinson, M., \& Giavalisco, M. 2000, ApJ, 528, 96

Pettini, M., Rix, S. A., Steidel, C. C., et al. 2002, ApJ, 569, 742

Prochaska, J. X., \& Wolfe, A. M. 1997, ApJ, 487, 73

Prochaska, J. X., Kasen, D., \& Rubin, K. 2011, ApJ, 734, 24 
Quider, A. M., Pettini, M., Shapley, A. E., \& Steidel, C. C. 2009, MNRAS, 398, 1263

Quider, A. M., Shapley, A. E., Pettini, M., Steidel, C. C., \& Stark, D. P. 2010, MNRAS, 402, 1467

Quiret, S., Péroux, C., Zafar, T., et al. 2016, MNRAS, 458, 4074

Rigby, J. R., Bayliss, M. B., Sharon, K., et al. 2018, AJ, 155, 104

Rudie, G. C., Steidel, C. C., Trainor, R. F., et al. 2012, ApJ, 750, 67

Salmon, B., Coe, D., Bradley, L., et al. 2017, arXiv:1710.08930

Sánchez-Ramírez, R., Ellison, S. L., Prochaska, J. X., et al. 2016, MNRAS, 456, 4488

Sanders, R. L., Shapley, A. E., Kriek, M., et al. 2015, ApJ, 799, 138

Sanders, R. L., Shapley, A. E., Kriek, M., et al. 2016, ApJL, 825, L23

Savage, B. D., \& Sembach, K. R. 1996, ARA\&A, 34, 279

Scoville, N., Aussel, H., Sheth, K., et al. 2014, ApJ, 783, 84

Scoville, N., Sheth, K., Aussel, H., et al. 2016, ApJ, 820, 83

Shapley, A. E., Steidel, C. C., Pettini, M., \& Adelberger, K. L. 2003, ApJ, 588,65

Sheinis, A. I., Bolte, M., Epps, H. W., et al. 2002, PASP, 114, 851
Stark, D. P., Auger, M., Belokurov, V., et al. 2013, MNRAS, 436, 1040

Steidel, C. C., Erb, D. K., Shapley, A. E., et al. 2010, ApJ, 717, 289

Steidel, C. C., Strom, A. L., Pettini, M., et al. 2016, ApJ, 826, 159

Tchernyshyov, K., Meixner, M., Seale, J., et al. 2015, ApJ, 811, 78

Thomas, D., Maraston, C., Bender, R., \& Mendes de Oliveira, C. 2005, ApJ, 621,673

Tomczak, A. R., Quadri, R. F., Tran, K.-V. H., et al. 2014, ApJ, 783, 85

Tremonti, C. A., Heckman, T. M., Kauffmann, G., et al. 2004, ApJ, 613, 898

Tumlinson, J., Thom, C., Werk, J. K., et al. 2011, Sci, 334, 948

Turner, M. L., Schaye, J., Steidel, C. C., Rudie, G. C., \& Strom, A. L. 2014, MNRAS, 445, 794

Vladilo, G., Centurión, M., Bonifacio, P., \& Howk, J. C. 2001, ApJ, 557, 1007

Werk, J. K., Prochaska, J. X., Thom, C., et al. 2013, ApJS, 204, 17

Wolfe, A. M., \& Prochaska, J. X. 2000, ApJ, 545, 591

Wuyts, S., Förster Schreiber, N. M., Wisnioski, E., et al. 2016, ApJ, 831, 149

Zhu, G., \& Ménard, B. 2013, ApJ, 770, 130

Zibetti, S., Ménard, B., Nestor, D., \& Turnshek, D. 2005, ApJL, 631, L105 